University of Louisville

ThinkIR: The University of Louisville's Institutional Repository

Electronic Theses and Dissertations

$12-2018$

\title{
Cholesterol metabolism and statin effects on an FH class II LDL- receptor mutation.
}

\author{
Linda Omer \\ University of Louisville
}

Follow this and additional works at: https://ir.library.louisville.edu/etd

Part of the Cardiovascular Diseases Commons, Medical Molecular Biology Commons, and the Other Medical Sciences Commons

\section{Recommended Citation}

Omer, Linda, "Cholesterol metabolism and statin effects on an FH class II LDL-receptor mutation." (2018). Electronic Theses and Dissertations. Paper 3080.

https://doi.org/10.18297/etd/3080

This Doctoral Dissertation is brought to you for free and open access by ThinkIR: The University of Louisville's Institutional Repository. It has been accepted for inclusion in Electronic Theses and Dissertations by an authorized administrator of ThinkIR: The University of Louisville's Institutional Repository. This title appears here courtesy of the author, who has retained all other copyrights. For more information, please contact thinkir@louisville.edu. 


\title{
CHOLESTEROL METABOLISM AND STATIN EFFECTS ON AN FH CLASS II LDL-RECEPTOR MUTATION
}

\author{
By \\ Linda Omer \\ B.S., Chicago State University, 2011 \\ M.S., Chicago State University, 2014 \\ M.S., University of Louisville, 2015

\begin{abstract}
A Dissertation
Submitted to the Faculty of the School of Medicine of the University of Louisville In Partial Fulfillment of the Requirements
\end{abstract} \\ For the Degree of \\ Doctor of Philosophy \\ in Biochemistry and Molecular Genetics \\ Department of Biochemistry and Molecular Genetics \\ University of Louisville \\ Louisville, KY
}

December 2018 
(C) 2018 by Linda Omer

All rights reserved 



\section{CHOLESTEROL METABOLISM AND STATIN EFFECTS ON AN FH CLASS II LDL-RECEPTOR MUTATION}

\section{By}

\section{Linda Omer}

B.S., Chicago State University, 2011

M.S., Chicago State University, 2014

M.S., University of Louisville, 2015

A Dissertation Approved on

November 9, 2018

By the following Dissertation Committee:

Nolan L. Boyd, Ph.D., Dissertation Director

Barbara J. Clark, Ph.D.

Alan Cheng, Ph.D.,

William L. Dean, Ph.D.

Ronald G. Gregg, Ph.D. 


\section{DEDICATION}

I dedicate this dissertation to my grandfather, Seedo Zakaria Hamad, who believed in my ability to speak truth before I knew I had a voice. He taught me of my Palestinian roots and our duty of continuous resistance to Israeli apartheid and occupation. He wanted me to become a politician but was even prouder when he saw me take a role in activism for human rights. I also dedicate this dissertation to my mother, Ustazah Abeer Salama Omer, who prioritized my education for freedom. She showed me the power only an immigrant mother can know. In $4^{\text {th }}$ grade, she did what a brown mother does and told me in her own handed way that I was going to do well in school and that I was going to succeed because she would support me through it all. Her resilience in the circumstances constantly put in her way is a reminder for me that I can make it through what may come my way. 


\section{ACKNOWLEDGMENTS}

Alhamdulillah (all praises and thanks be to Allah). Allah Al-Lateef that guided me throughout these years, showing me the righteous path, the one of mindfulness, gratitude, and wad (love). Jazak'allahu khayran to my mother, Abeer, my sisters and brothers, Hidaia, Sabreen, Nagieh, Tamam, Jenna, Mohamad, Ahmad, and Yominah, and my immediate family Khalto Maryam, Uncle Z, Rheann, Joad, Reneen, Adam, Saja, Khaled, and my brothers-in-law. Alhamdulillah for my Louisville family who have integrated me into the community, teaching me of social justice, vulnerability, and love:

Sister's Halaqa, Students for Justice in Palestine, Louisville Committee for Peace in the Middle East, and Stand Up Sunday. Thank you to my graduate advisor, Dr. Nolan Boyd. He is a born teacher who is patient, firm, and creates an environment for cultivation rather than harm. Thank you to my committee members, Dr. Gregg for his comments and lab support, Dr. Clark for the never-ending assistance, Dr. Dean for his words of kindness and support, and Dr. Cheng for our interesting conversations. Thank you to my former committee member Dr. Jay Hoying for assistance and encouragement. Thank you to Jason Beare, Katilin Stivers, Dr. Paula Chilton, and Dr. Lucasz Slomnicki for kindly always taking time to help. Thank you to Dr. Giusipeppe Militelo for his extensive assistance in optimizing experiments. Thank you to my current and former lab mates, Lubna Hindi, Dr. Shirong Zheng, and Lawal Alhassan for keeping it real. Thank you to Dr. Christine E. Schaner-Tooley for the mentorship and kindness 
she provided in my first few years in the program. I would like to thank the Department of Biochemistry and Molecular Genetics' professors and (former) students for the company, support, and laughs throughout these years. Thank you to Janice Ellwanger Burkett for keeping our department functioning. Deepest thanks to my companions during this journey Imani Thiongo, Elizabeth Hudson, Chaz Briscoe, Douglas Saforo, and Hamza Foy whose camaraderie, deep discussions, and hiking trips grounded me at the most necessary times. I am grateful to my mentors, Dr. Ibrahim Imam and Patricia Grier for their genuine care and counseling as I made my way into adulthood. Special thanks to my sister, Nagieh, for assistance in editing this dissertation. Finally, love and gratitude to my youngest and smallest masjid friends (and Yominah) who keep me motivated and young in heart. 


\title{
ABSTRACT \\ CHOLESTEROL METABOLISM AND STATIN EFFECTS \\ ON AN FH CLASS II LDL-RECEPTOR MUTATION
}

\author{
Linda Omer
}

November 9, 2018

Familial hypercholesterolemia $(\mathrm{FH})$ is a common genetic disease and has been studied with the aim of finding a curative measure for decades. FH is caused by mutations in the low-density lipoprotein receptor (LDLR) resulting in defects in LDLcholesterol (LDL-C)-receptor mediated endocytosis and development of premature cardiovascular disease (CVD). Here I describe the use of a corrected and non-corrected LDLR FH cell model to investigate receptor-mediated endocytosis and statin effects.

For these studies, we reprogrammed FH fibroblast cells to induced pluripotent stem cells (iPSC) and confirmed their pluripotency and ability to differentiate to hepatocyte-like cells (HLC). A clustered regularly interspaced short palindromic repeats (CRISPR)/CRISPR-associated 9 (Cas9) design was created and used to successfully correct a 3 base pair deletion in the class II LDLR FH iPSC. Western blot and immunocytochemistry analysis showed maturation and proper localization of the LDLR after correction in iPSC and HLC. We used internalization analyses to detect LDL-C receptor mediated endocytosis in non-corrected and corrected HLC. Real time PCR for ER stress markers showed that statin-induced accumulation of an immature LDLR in non-corrected FH cells did not activate the unfolded protein response. Together, this 
work demonstrates the capacity to utilize the resources of CRISPR, iPSC, and HLC to study misfolded class II LDLR providing a physiologically relevant in vitro model for investigating the differential effects of mutant versus corrected LDLR-mediated metabolism and current treatments like statins. 
TABLE OF CONTENTS

PAGE

DEDICATION

iii

ACKNOWLEDGMENTS

iv

ABSTRACT

vi

LIST OF TABLES

LIST OF FIGURES

CHAPTER I: BACKGROUND/LITERATURE REVIEW

PART I: Familial Hypercholesterolemia and the LDLR 1

PART II: Genome Editing and CRISPR/Cas9 25

PART III: The Endoplasmic Reticulum and Unfolded Protein Response 46

CHAPTER II: CRISPR Correction of a Homozygous LDLR Mutation in

Familial Hypercholesterolemia Induced Pluripotent Stem Cells

INTRODUCTION 52

MATERIALS AND METHODS 54

$\begin{array}{ll}\text { RESULTS } & 67\end{array}$

$\begin{array}{ll}\text { DISCUSSION } & 87\end{array}$

CHAPTER III: Mammalian Species Wnt3a Differential Modulation of Derived-

Definitive Endoderm

$\begin{array}{ll}\text { INTRODUCTION } & 93\end{array}$

MATERIALS AND METHODS 96

$\begin{array}{lr}\text { RESULTS } & 100\end{array}$

$\begin{array}{ll}\text { DISCUSSION } & 106\end{array}$ 
CHAPTER IV: Statin-Induced Accumulation of Class II Immature LDL-Receptor in

Familial Hypercholesterolemia Does Not Activate the Unfolded Protein Response $\begin{array}{ll}\text { INTRODUCTION } & 109\end{array}$

MATERIALS AND METHODS 111

$\begin{array}{ll}\text { RESULTS } & 117\end{array}$

$\begin{array}{lr}\text { DISCUSSION } & 134\end{array}$

CHAPTER V: SUMMARY AND CONCLUSIONS 140

$\begin{array}{ll}\text { REFERENCES } & 144\end{array}$

$\begin{array}{ll}\text { CURRICULUM VITAE } & 181\end{array}$ 


\section{LIST OF TABLES}

TABLE

Table 1: $\quad$ Chapter II: List of Antibodies/Fluorophores 64

Table 2: $\quad$ Chapter II: Primers for qPCR 65

Table 3: $\quad$ Chapter II: Guide Design and Primers 65

Table 4: $\quad$ Chapter II: GFP Primers $\quad 65$

Table 5: Chapter II: Electroporation Conditions 66

Table 6: $\quad$ Chapter II: Off-Targets 66

Table 7: $\quad$ Chapter III: List of Antibodies in FACS 99

Table 8: $\quad$ Chapter III: Primers for qPCR analysis of Definitive 99

Endoderm

Table 9: $\quad$ Chapter IV: Primers for LDLR and ER Stress 116

Table 10: $\quad$ Chapter IV: Antibodies for ICC and Western Blot 117 


\section{LIST OF FIGURES}

FIGURE PAGE

Figure 1: Schematic Representation of the Human LDLR Promoter, 14 Gene, Domain, and Protein

Figure 2: Schematic of Human LDLR-Receptor Mediated Endocytosis

Pathway

Figure 3: Representation of Tools and their Mechanisms for Genome Editing and DSB Repair

Figure 4: CRISPR/Cas9 and its Modification for Altering the Genome 40

Figure 5: Schematic of the Unfolded Protein Response (UPR) 50

Figure 6: Mutation Identification in Homozygous FH Fibroblasts 69

Figure 7: GM03040 are Karyotypically Normal 71

Figure 8: Reprogrammed 3040-iPSC are Pluripotent 72

Figure 9: Hepatocyte-Like Cells (HLC) can be Derived from 3040-iPSC 74

Figure 10: CRISPR Correction Strategy Methodology Schematic 76

$\begin{array}{ll}\text { Figure 11: CRISPR ssODN Design } & 77\end{array}$

$\begin{array}{ll}\text { Figure 12: CRISPR Positive Sorting } & 79\end{array}$

Figure 13: Genetic Analysis 3040-iPSC LDLR Correction by CRISPR/Cas9 82

Figure 14: Protein Analysis 3040-iPSC LDLR Correction by CRISPR/Cas9 85

Figure 15: LDLR-Mediated Endocytosis is Restored in Corrected 86 3040-iPSC \& HLC

Figure 16: Hepatocyte-like cell (HLC) Differentiation Protocol 97

$\begin{array}{ll}\text { Figure 17: Differentiation for Endoderm } & 102\end{array}$ 
Figure 18: All Protocols Result in a Mixed Population of Tra-1-81 and CXCR4 104

Figure 19: Human Wnt3a Causes Increased Hnf4a Expression Compared 105 to Mouse Wnt3a

Figure 20: Hnf4a Expression Occurs by Day 5 in Endoderm Differentiation 106

Figure 21: LDLR-Mediated Cholesterol Internalization is Restored in C-HLC 120

Figure 22: LDLR Colocalizes with Calnexin-ER in FH-HLC. 123

Figure 23: Rosuvastatin Increases Total LDLR Protein Levels and an 128 Accumulation of Immature LDLR in NC-cells.

Figure 24: FH-NC cells Do Not Activate the UPR Following Rosuvastatin Treatment. 


\section{CHAPTER I}

\section{BACKGROUND/LITERATURE REVIEW}

\section{PART I: FAMILIAL HYPERCHOLESTEROLEMIA AND THE LDLR}

\section{Familial Hypercholesterolemia (FH)}

High serum cholesterol levels are correlated with an increased risk in the development of cardiovascular disease. A subset of the population cannot maintain cholesterol levels without medical interventions due to genetics.

Familial hypercholesterolemia (FH) is the most common genetic disease in our population, affecting 1:250 people (heterozygous FH, HetFH) [1-3]. As early as 1938, Dr. Carl Muller observed "an inborn error of metabolism" or genetic mutation he used to describe young patients with both high cholesterol and myocardial infarctions [4]. He labeled FH as a single gene-determined autosomal dominant trait. Two decades later, researchers studying populations with high incidence of $\mathrm{FH}$ believed to be due to a founder's affect confirmed clinically both the HetFH and homozygous $\mathrm{FH}(\mathrm{HoFH})$ disease. Still then, it was unknown what mutation was causing this hypercholesterolemia.

Cholesterol is obtained by diet and liver synthesis [5]. Intestinal mucosal cells convert dietary cholesterol into lipoproteins. A lipoprotein is a micelle-like structure comprised of a nonpolar core containing triacyclglycerols and cholesterol esters surrounded by a amphiphilic proteins and cholesterol. There are five classes of lipoproteins categorized by density and function: chylomicrons, very low density lipoprotein (VLDL), 
intermediate density lipoprotein (IDL), low density lipoprotein (LDL), and high density lipoprotein (HDL) [5]. The protein component of the lipoprotein is the apoprotein. Our lipoprotein of interest, LDL, contains a single apolipoprotein B-100 (apoB-100) molecule that covers half the particle surface [5]. Because LDL is the major carrier of cholesterol, it is commonly referred to as LDL-cholesterol (LDL-C).

The levels of cholesterol are tightly regulated to maintain balance between biosynthesis, utilization, and transport of cholesterol to prevent its accumulation and deposition in arteries [6]. Briefly, cholesterol biosynthesis begins when acetate (from acetyl-CoA) is converted to isoprene units containing a 5-carbon skeleton. These isoprene units will eventually condense to form a linear molecular with 30 carbons that cyclizes to form the cholesterol molecule [6]. The process begins in the liver where acetyl-CoA is converted to 3-hydroxy-3-methylglutaryl-CoA (HMG-CoA) by HMG-CoA synthase and thiolase. The next step is the rate-limiting step of cholesterol biosynthesis. HMG-CoA reductase will catalyze the reaction of HMG-CoA to mevalonic acid which will proceed through an additional 8-12 steps for the production of cholesterol [6]. HMG-CoAreductase is the enzyme involved in the rate-limiting step in cholesterol synthesis, and is regulated through feedback control where the amount of enzyme present in the cell can increase or decrease based on the amount of cholesterol in the cell [7]. HMG-CoA is also controlled by competitive inhibition, allosteric effects, and covalent modifications that will change it from active and less active states [4]. Statins competitively inhibit HMG-CoA reductase activity with a greater $\mathrm{K}_{\mathrm{m}}$ than its $\mathrm{HMG-CoA}$ substrate $[8,9]$.

In the early 1970s studies on human livers were nearly impossible making it difficult to study cholesterol metabolism [10]. To overcome this limitation, investigation 
using skin fibroblast cells from patients with FH because they were known to express the inherited mutation. Normal and FH affected skin fibroblasts were treated with lipoprotein deficient serum (LPDS) with or without additional LDL-C [4, 11]. They HMG-CoAreductase activity to determine activity in FH patient fibroblasts [11, 12]. In the presence of serum, normal fibroblasts had low HMG-CoA-reductase activity while LPDS media resulted in a 50-fold increase in HMG-CoA-reductase activity. In addition, they noticed the addition of as low a concentration as $5 \mu \mathrm{g} / \mathrm{mL}$ LDL would decrease HMG-CoAreductase activity [11]. These results allowed them to hypothesize that a receptor might be regulating cholesterol uptake since LDL and its concentration were necessary to see changes in enzymatic activity of HMG-CoA-reductase.

In fibroblasts from HoFH patients, there was a 50-100-fold increase in HMG-CoAreductase activity even in the presence of lipoproteins [11], and the addition of LPDS with or without LDL had no effect on HMG-CoA-reductase activity. This led to their hypothesis that HMG-CoA-reductase may be the site of a mutation, causing a break in the feedback loop of LDL-C metabolism and hence FH. However, upon the addition of ethanoldissolved cholesterol to either normal or affected FH skin cells, enzymatic activity was suppressed uniformly $[7,11]$. This indicated that HMG-CoA-reductase was not responsible for the phenotype of FH.

To try to solve this mystery, Brown and Goldstein turned to the use of radioiodine labeled LDL-C ( ${ }^{125}$ I-LDL) and hypothesized that a cell surface receptor must be present and responsible for the uptake of LDL-C. Normal and FH fibroblasts were incubated in ${ }^{125}$ I-LDL to measure binding $[7,13]$. Normal human fibroblasts bound and degraded ${ }^{125}$ ILDL and at a high rate when ${ }^{125}$ I-LDL was present at a low concentration $[7,13]$. This is 
correlated with a high rate of degradation. However, upon incubation with high levels of ${ }^{125}$ I-LDL, the binding decreased and the rate of bound/degraded was similar in both normal and FH fibroblasts. It was hypothesized this was due to receptor independent endocytosis. In the presence of low or high concentrations of ${ }^{125} \mathrm{I}-\mathrm{LDL}, \mathrm{HoFH}$ fibroblasts had low binding and degradation [7, 13]. Further studies were done to differentiate between internalized or surface bound ${ }^{125} \mathrm{I}$-LDL. Techniques involved experiments at $4^{\circ} \mathrm{C}$ and $37^{\circ}$ $\mathrm{C}$ and the sequential use of buffers that would allow the measurement of bound versus internalized ${ }^{125}$ I-LDL. It was discovered that ${ }^{125}$ I-LDL-bound to a receptor and remained so for no more than 10 minutes $[7,13]$. This followed with complete catabolism of ${ }^{125}$ ILDL into its components of amino acids and free fatty acids within an hour. With the chloroquine inhibitor and lysosomal defective patient fibroblasts, it was confirmed this rapid degradation occurred within the lysosomal vesicle [7, 13]. The free fatty acids released by the lysosome then went on to act as an inhibitor of HMG-Co-A reductase, inhibiting its activity and therefore cholesterol production [7, 13]. This data clearly demonstrated the definite presence of a cell surface receptor that was responsible for the binding and endocytosis of LDL-C [7, 13].

Although most FH is caused by mutations of the low-density lipoprotein receptor (LDLR), defects in the apolipoprotein B (ApoB), proprotein convertase kinase 9 (PCSK9), and the low-density lipoprotein receptor adaptor protein $1(\mathrm{ARH})$ genes are also responsible for this disease [14]. This inherited disease can be transmitted through due to a single (HetFH) or two defective alleles $(\mathrm{HoFH})$ leading to a range in the development of cardiovascular disease. Normal cholesterol levels should be less than $100 \mathrm{mg} / \mathrm{dL}$. As levels increase, a person can be considered hypercholesterolemic if levels reach between 150-200 
$\mathrm{mg} / \mathrm{dL}$. HetFH patients are common (1:250) can have LDL-C levels from 200-500 mg/dL. The rarer HoFH is present in 1:250,000 people and leads to cholesterol levels as high as $1000 \mathrm{mg} / \mathrm{dL}$ [1-3]. Because of the extremely elevated cholesterol levels, apparent symptoms can arise that include cutaneous xanthomas and corneal arcus [14]. HoFH patients especially present with murmurs of aortic stenosis and symptoms of ischemic heart disease in early childhood [15] which can result in death due to myocardial infraction before the age of 25 if left untreated [15-18].

HetFH is treatable through strict lifestyle requirements such as exercising regularly, consuming a low saturated and trans-fat diet, taking medications, and using lipid apheresis [15, 19-21]. Several non-curative pharmacologic treatments are available, while new ones have been recently approved for FH patients [22-24]. The well-known and most prescribed drug is statin [25]. Statins are competitive inhibitors of HMG-CoA reductase and therefore (1) inhibit endogenous cholesterol production and (2) upregulate LDLR expression [25]. This process forces the cell to scavenge LDL-C that is present in circulation through the increased LDLR in a process called LDLR-mediated endocytosis resulting in decreased circulating LDL-C levels $[9,25]$.

Although statin treatment has a significant therapeutic benefit in HetFH patients, most HoFH patients receive minimal benefit $[2,15,26]$. A non-functional/receptordefective/negative patient will be required to supplement their diet with high dose statins along with other drugs and alternative treatments such as Mipomersin [2, 15, 26]. Mipomersin acts similarly to microRNAs [24]. It is an antisense oligonucleotide that binds to the mRNA sequence of ApoB-100, promoting ApoB's mRNA degradation by ribonuclease $\mathrm{H}$ [24]. By reducing ApoB-100 production, very low-density lipoprotein 
(VLDL) generation by the liver is also reduced, consequently decreasing circulating LDLC levels. An emerging therapy for FH is the use of monoclonal antibody to PCSK9. PCSK9 binds to LDLR, accelerating its degradation [27-29]. Within the human population exist mutations for PCSK9 loss-of-function that leads to an $88 \%$ reduction in coronary artery disease (CAD) [27]. PCSK9 binds in the epidermal growth factor (EGF)-A domain of LDLR, and mutation of an aspartate residue $\left(\mathrm{Asp}^{310}\right)$ in LDLR inhibits binding and subsequent degradation of LDLR by PCSK9 [28]. Monoclonal antibodies like Evolocumab target PCSK9 and are 70\% more effective in lowering LDL-C than statins [30]. However, receptor negative $\mathrm{HoFH}$ patients did not respond to Evolocumab since there is no plasma membrane LDLR to target [23]. There was a large variation in response to Evolocumab in 2 genetically identical homozygous receptor-defective patients. Both began with similar baseline LDL-C levels; one patient showed the largest decrease in LDL-C levels while the other had the smallest decrease in LDL-C post-clinical trial [22]. Furthermore, statins have been shown to upregulate PCSK9, reducing LDLR levels and decreasing statins' efficacy in the more prevalent HetFH [31].

An alternative treatment for HoFH patients that do not respond to medicinal treatments is the dialysis-like lipid apheresis $[15,19]$. The aim of lipid apheresis is to reduce plasma LDL-C levels to prevent the progression of premature CAD. The technique involves "plasma exchange" in which plasma LDL-C is selectively removed without affecting other proteins present in the serum [19, 32]. Lipid apheresis is beneficial in having shown over a $60 \%$ decrease in LDL-C levels. However, upon removal from apheresis, the cholesterol levels rebound rapidly [19]. Although this can be the only standard of treatment for some patients with $\mathrm{HoFH}$, it is limited in availability, requires frequent trips for dialysis 
that can cost upwards of $\$ 250,000 /$ year, and presents patient complication, such as venous access [19].

A liver transplant is the only currently definitive cure for $\mathrm{FH}$, but also requires lifelong immunosuppressive drugs $[3,33,34]$. It is not the first treatment option, but rather a secondary prevention for patients with severe HoFH [34-36]. One study reported that after treating $36 \mathrm{FH}$ patients with liver transplants the key to successful liver transplantation is timing of transplant [33]. In the study, the majority of patients had LDL-C levels greater than $500 \mathrm{mg} / \mathrm{dL}$, and 11 of these patients were under 8 years old [33]. When treatment with high dose statins had failed to decrease LDL-C levels, a liver transplant was considered for treatment. After liver transplantation, the patients were followed up from six months to six years. Three patients under the age of four years died due to cardiac arrest or septicemia [33]. Most of the patients showed a regression of xanthomas and normalization of LDL-C levels. A seven-year-old boy with severe HoFH presented a drastic reduction in LDL-C from $946 \mathrm{mg} / \mathrm{dL}$ to $71 \mathrm{mg} / \mathrm{dL}$ post-liver transplant; however, he still required an aortic root replacement [37]. The current practice is to treat patients with the maximal dose of statins and monitor until the necessity for a liver transplant occurs because of the onset of CVD. This case helps argue that the timing of transplantation is key to a successful liver transplantation, rather than following the current practice $[33,37]$.

An experimental treatment approach for FH involves gene therapy that uses a vector to deliver a functional LDLR to the liver [3]. Gene delivery can be performed ex vivo, in vitro, and in vivo. Ex vivo gene therapy [38] was one of the first processes used for gene therapy [3] and included harvesting the liver resection tissue from an FH patient, isolating the autologous hepatocytes, growing the cells in vitro, introducing the LDLR into 
the cells using a recombinant retrovirus, and delivering the now genetically modified cells back into the FH patient $[39,40]$. Studies using the Watanabe heritable hyperlipidemic rabbit (WHHL), which is considered an authentic model of FH [41], demonstrated that transplantation of both normal or genetically altered allogenic hepatocytes could lower serum LDL-C in WHHL rabbits. However, the decrease was temporary with the high number of rejected allogenic cells likely due to an immune response [42-44]. Instead, Wilson and colleagues focused on using autologous retroviral-LDLR expressing hepatocytes transplanted back into the WHHL rabbit. The results were a prolonged improvement in serum cholesterol levels with liver tissue showing a high level of recombinant derived LDLR RNA expression throughout the duration of the six and a halfmonth experiment [45].

The success of liver-based gene therapy is largely dependent on the method of gene delivery. The most efficient method used thus far has been through viral vectors [46-49]. Wilson's group found that the delivery of 0.1 to 1 proviral copy of the LDLR-expressing retrovirus into isolated human hepatocytes resulted in LDLR protein levels exceeding the normal endogenous levels of the human hepatocytes [40]. In addition, the infection efficiency was highest when the cells were exposed to the virus 48 hours post plating, allowing gene transduction to be achieved within 72 hours after initial seeding, minimizing the time the hepatocytes were in culture prior to transplantation [40].

When the technique appeared to be successful in the animal models, Wilson moved onto clinical trials with FH patients. The trial involved a receptor-defective HoFH patient who underwent liver resection followed by transplantation of the genetically modified autologous hepatocytes [50]. The patient had a 17\% decrease in plasma LDL-C levels after 
a 25\% hepatectomy and re-infusion. She also did not show any immunogenic response to the viral vector. This study demonstrated the feasibility, safety, and potential efficacy for ex vivo gene therapy in humans [50]. After 2.5 years, the patient still maintained a $20 \%$ decrease in serum LDL-C levels. However, because the patient was receptor defective but had a partially functional LDLR to begin with, there were questions in regard to whether patients with different genotypes, including receptor negative LDLR, would respond to this treatment. The first pilot study for liver-directed gene therapy to be done in $\mathrm{HoFH}$ patients included patients ranging in age from 7 to 41 years old and with mutations that resulted in receptor defective and receptor negative phenotypes [51]. From the studies, a 12-year-old receptor negative $\mathrm{HoFH}$ patient showed a significant reduction in serum LDL-C levels post gene transfer. Another two patients also showed prolonged reduction in LDL-C levels 4 months after therapy. However, two patients failed to show any significant changes in LDL-C levels. The variability in results led to a halt in the clinical trials [51]. Of importance, the isolation and survival of primary hepatocytes for this process was essential for the successes seen in the trials. Primary hepatocytes cannot be passaged; therefore, transduced cells cannot be selected and expanded $[49,52,53]$. The cells also remain differentiated and viable for only a short period of time; Wilson's experiment showed that infecting cells within 48 hours of isolation showed an open window in which the cells were most susceptible to infection [54].

There are still barriers preventing successful gene therapy including 1) vector genome persistence in which the expression can vary depending on if the DNA exists as an episomal molecule or integrative in the host, 2) loss of gene expression due to immunogenicity to the transduced cells or gene product, and 3) their uptake and transport 
requires tissue specificity but this can be influenced by the organ's vascular supply and endothelial barriers $[53,55,56]$. In addition, their delivery usually requires the stimulation of hepatocyte proliferation through either hepatectomy or cytotoxic agents [57-60]. Gene correction through gene targeting could provide an alternative approach to correcting the mutation in vivo without affecting the rest of the genome.

\section{$\underline{\text { Statins }}$}

With the link finally made between risk of heart disease and cholesterol levels, many researchers began their focus on finding a molecule inhibitor of any of the 30 steps involved in the synthesis of cholesterol $[61,62]$. Of the many inhibitors synthesized and tested, only one made it to the market, Triparanol (MER/29). MER/29 inhibited 24dehydrocholesterol reductase preventing the conversion of desmosterol to cholesterol [63, $64]$.

Since MER/29 inhibited the enzyme that catalyzed the final step to cholesterol, it caused an accumulation of cholesterol intermediates resulting in severe side effects including vision loss due to irreversible cataracts, accelerated atherosclerosis, and even alopecia $[65,66]$. At this time, other groups studying enzymes discovered the ability of antibiotics to inhibit enzymatic activity. An antibiotic that inhibits HMG-CoA-reductase in microbes could be lethal to them and successful in mammals. This led Akira Endo's group to hypothesize that fungi like molds and mushrooms could potentially produce antibiotics that inhibit the rate-limiting step in cholesterol synthesis thru HMG-Co-A reductase and thus, be lethal to these microbes [67]. His group utilized radiolabeled ${ }^{14} \mathrm{C}$ acetate active microbial culture broths. The concept was that broths that inhibited the incorporation of ${ }^{14} \mathrm{C}$-acetate into nonsaponifiable (or non-ester bonded) lipids could be 
tested for their ability to inhibit lipid synthesis from ${ }^{3} \mathrm{H}-$ mevalonate. If a culture broth was inactive, it was suspected to contain inhibitory compounds that prevented the early stages of cholesterol synthesis from acetate to mevalonate. The isolated compounds were tested on rat liver enzymes [67-69].

Over one year and 3800 fungal strains later, Endo found a mold culture broth containing citrinin [70]. Citrinin inhibited HMG-CoA-reductase and even lowered serum cholesterol levels in rats [71]. However, the molecule also caused kidney toxicity [71]. Eventually, his group found an active culture of blue-green mold, Penicillium citrinum Pen51, isolated from a rice sample. They isolated 3 active metabolites through solvent extraction, silica gel chromatography, and crystallization [72]. Compactin (ML-236B) was the most active and structurally similar to HMG-CoA. Because of this, compactin was an extremely effective competitive inhibitor for HMG-CoA-reductase [8]. Unless cells were homogenized to dilute compactin in vitro, no HMG-CoA-reductase activity could be detected. However, when rats were fed compactin 7 days in a row, there was no reduction in serum cholesterol [73]. The caveat of compactin is its ability to lower cholesterol but only for 3 to 8 hours after a single dose. After 8 hours, an almost 10-fold induction of hepatic HMG-CoA-reductase activity would occur [67, 73]. Endo's findings suggested compactin would still be beneficial but in experimental animals with elevated blood cholesterol levels. They showed that in chickens, dogs, and monkeys compactin treatment decreased plasma cholesterol levels after a month [67]. With the collaboration of Dr. Akira Yamamoto at Osaka University Hospital, compactin was used to treat an 18-year-old female FH patient who had serum cholesterol levels of $1000 \mathrm{mg} / \mathrm{dL}$. Within two weeks of a daily dose of $500 \mathrm{mg}$ compactin, her serum cholesterol levels dropped to $700 \mathrm{mg} / \mathrm{dL}$. 
However, she developed muscular dystrophy like symptoms and elevated trans-aminase that were reversed upon discontinuation of the high dose of compactin [67, 74]. Reducing her dose to $200 \mathrm{mg}$ still resulted in reduced tuberous and Achilles tendon xanthomatosis, but no reduction in serum cholesterol levels. Nevertheless, clinical trials continued with 5 HetFH patients who showed an on average $30 \%$ reduction in serum cholesterol levels with no adverse side effects $[67,74]$.

Many pharmaceutical industries set out to find molecular inhibitors similar to compactin. Merck, upon signing a confidentiality agreement with Sankyo, obtained both samples of compactin and the experimental data of Endo [67]. With this resource, Merck's scientists developed a structurally similar drug to compactin called mevinolin from the fungus Aspergillus terreus [75]. The two compounds differed by only one methyl group and displayed similar biological properties $[75,76]$. Endo continued his work at Tokyo Noko University and isolated another statin named monacolin K from Monascus ruber. Surprisingly, monacolin $\mathrm{K}$ and mevinolin were the same compound and would be renamed Lovastatin [77].

Clinicians began treating patients with severe hypercholesterolemia with Lovastatin and saw their LDL cholesterol levels decrease dramatically with minor side effects [78]. In 1986, the Food and Drug Administration approved Lovastatin as the first commercially available statin [78]. Since then, another 6 statins have been developed. Simvastatin and Pravastatin are semi-synthetic statins that are Lovastatin with either an extra methyl group or hydroxyl group, respectively [25]. Four synthetic statins have subsequently been developed including Fluvastatin, Rosuvastatin, Pitavastatin, and the currently popular Atorvastatin [67]. Many large scale clinical trials, including a five-year 
study, have all shown the ability of statins to lower LDL-cholesterol and decrease the incidence of CVD and stroke by $30 \%$ [67]. It is estimated that over 30 million people globally take statins generating over $\$ 25$ billion in revenue [67]. Ideal for modern day medicine, a difficulty of statins is the ability to predict an individual's response and genetic predispositions that cause adverse side effects. For example, a study has shown a 45 -fold variability in plasma Atorvastatin and Rosuvastatin concentration, in patients receiving the

same dose [9, 79]. Major genetic variants have been linked to both the variability in concentration as well as serious side effects seen in certain populations, requiring further research to determine statin efficacy and safety on a person-to-person level [9]. Interestingly, besides statins primary role in lowering lipids levels, it has also become an imperative treatment for the intervention in coronary artery and vascular disease [80, 81], rheumatoid arthritis, polycystic ovary syndrome, sepsis, and cancer [81-86].

\section{$\underline{\text { Low Density Lipoprotein-Receptor (LDLR) }}$}

The LDLR is a cell-surface endocytic receptor responsible for the internalization and regulation of LDL-C $[4,87]$. The mature LDLR is an 839-amono acid type I transmembrane glycoprotein encoded by 18 exons [88, 89]. These 18 exons code for 5 functional domains (Figure 1). 


\section{Promoter}
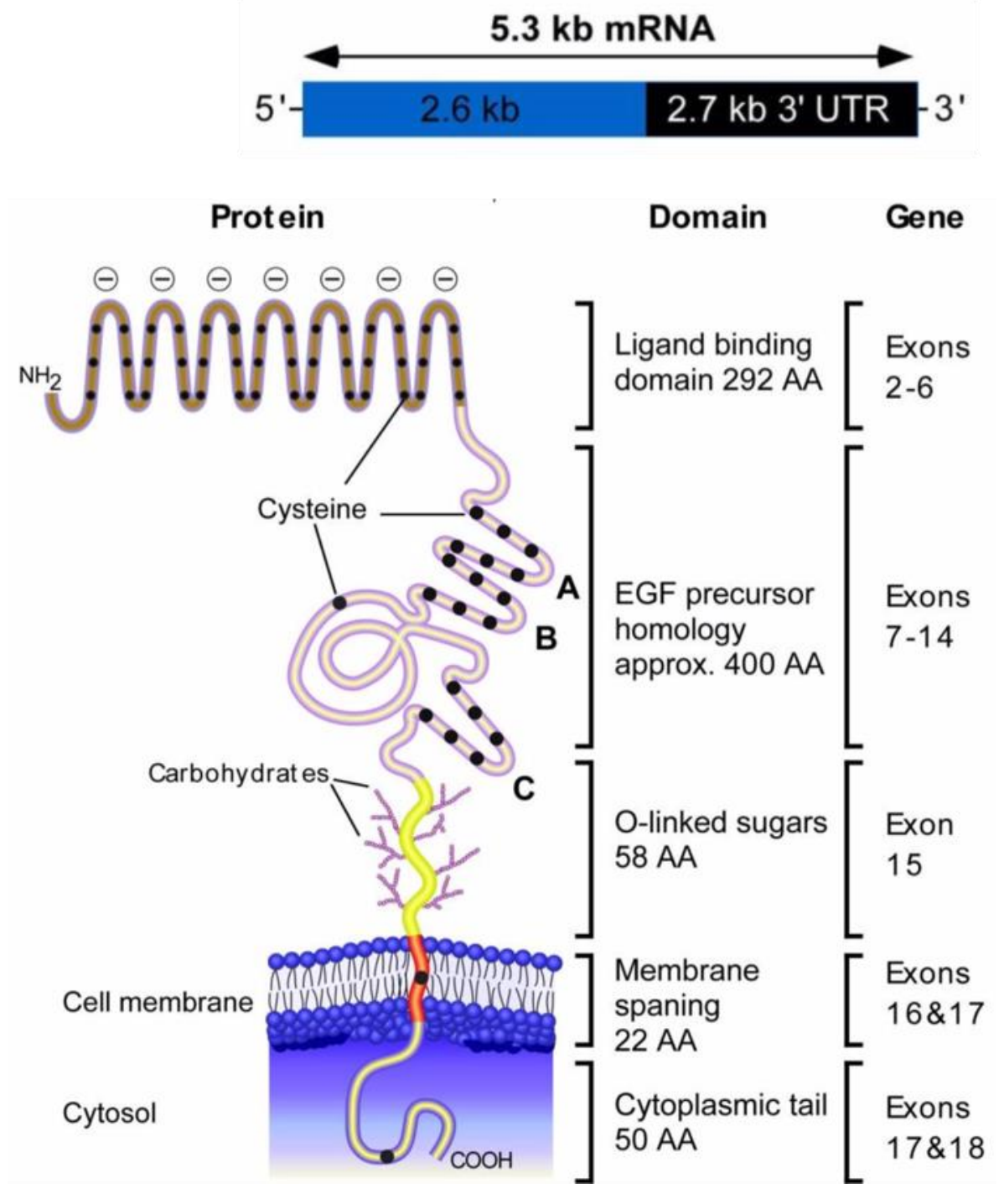

Figure 1. Schematic Representation of the Human LDLR Promoter, Gene, Domain, and Protein Structure. The mature LDLR is made up of 18 exons that code for 5 functional domains to make up a cell surface endocytic receptor. Mutations have been found in the promoter region and the 18 exons leading to dysfunction LDLR structure and/or function. Adapted and modified from Faisal A. Al-Allaf et al. Int Arch Med. 2010; 3: 36. [90]

The promoter region for LDLR is made up of three cis-acting DNA sequences, which have been found to allow for the ubiquitous and regulated expression of the LDLR gene [91, 92]. Repeats 1 and 3 interact with a general factor, $\mathrm{Sp} 1$, to promote transcription [91, 92]. Repeat 2 contains a conditional-positive sterol regulatory element (SRE-1) that is 
necessary for sufficient high-level expression of LDLR. If sterols are absent, SRE-1 synergizes with the two Sp1 sites to promote LDLR transcription. The presence of sterols inactivates SRE-1, suppressing LDLR transcription and hence surface LDLR expression $[4,93,94]$.

Exon 1 encodes a short 21 amino acid signal sequence for the LDLR that is cleaved upon translocation to the ER $[92,95]$. The ligand-binding domain is encoded by exons 26. This domain is made up of 7 repeats termed the LDLR type A repeat (LR). LR's 1, 2, 6, and 7 are each coded by a single exon while LR's 3-5 are encoded by a single exon. The LR's are 40 amino acids long and contain 6 cysteine residues that form three intra-repeat disulfide bonds $[92,95]$. The C-terminus of the LR's also contain a negatively charged repeat of Ser-Asp-Glu that is important for Apo B-100, Apo-E, and calcium binding to the LDLR. Of interest, these LR's have homology to the C9 protein of the complement cascade involved in the immune response. The EGF precursor domain is encoded by exons 7-14 and is made up of 400 amino acids. This region contains 3-40 amino acid cysteine rich repeats or $33 \%$ sequence identity to the human EGF precursor gene. These cysteine rich repeats are different than those found in the LR of the ligand binding domain. The first two repeats, EGFP-A and EGFP-B, are contiguous and are separated from the third repeat (EGFP-C) by 280 amino acids. This separation sequence contains 5 copies of a conserved YWTD (tyrosine, tryptophan, threonine, and aspartate) repeat every 40-60 amino acids. EGFP-A's role is special in that it interacts in a sequence-specific manner with PCSK9 [28, 96] leading to the early degradation of the LDLR. Otherwise, these EGFP-domains are required for the acid-dependent disassociation of the lipoproteins from the receptor in the endosome [92]. Exon 15 encodes for an enriched serine and threonine 58-count amino 
acid sequence. These act as sites for the attachment of O-linked carbohydrates. Deletion of exon 15 has not been shown to have any functional consequences in cultured hamster fibroblasts [97]. The 22 hydrophobic amino acids of the transmembrane or anchor domain of the LDLR is encoded by exon 16 and the 5' end of exon 17 . The last 50 amino acids make up the cytoplasmic domain and are encoded by the rest of exon 17 and exon 18. This region contains an NPxY motif necessary for the localization of the receptor into clathrincoated pits as well as the sorting of the receptor to the basolateral membrane in polarized cells [87].

In normal cells, the LDLR is transcribed and the mRNA is translated in the ER where the newly synthesized protein is properly folded into its immature 120-kDa form. The manner in which the domains fold into a functional LDLR is still unclear. Interestingly, the LDLR has a structure that is vectorially organized — each domain is sequential to the next. Even so, it has been shown that the N-terminal repeats will not attain their native conformation until 30 minutes after chain termination [98]. Studies using electrophoretic mobility assays in combination with metabolic radiolabeling revealed that instead the newly synthesized LDLR polypeptide chains folded rapidly into a compact structure with creating random disulfide bond interactions connecting distant regions of the LDLR [99, 100]. Through isomerization, the random disulfide bonds rearranged themselves, allowing extension of the LDLR until the native conformation is formed and the only disulfide bonds present are between the cysteine residues within each individual LDLR type A repeat [99, 100]. Interestingly, even when undergoing the extensive formation of non-native disulfide bonds during its folding, the LDLR seldom aggregates [98], which has led to the assumption that non-native disulfide bonds isomerization process is originally part of the 
folding of the LDLR. Calcium also plays a significant role in the proper folding and function of the LDLR. The cysteine repeats in both the ligand binding domain and the EGFP-A and EGFP-B regions have high affinity for calcium ions. Once the native disulfide bonds have formed, calcium binds between distal disulfide bonds. This is especially critical in the EGFP regions since the calcium ion stabilizes the linker region between the repeats and maintains their fixed orientation to each other $[101,102]$.

Of course, the proper folding of the LDLR could not be as efficient as it is without the assistance of chaperones. Chaperones are important in maintaining newly synthesized proteins in a protected environment until its disulfide bonds are formed. Disulfide bonds are usually formed in a vectorial manner unless the $\mathrm{N}$ and $\mathrm{C}$-termini are in close proximity in their tertiary structure. The most well-known and described is Grp78 for glucoseregulated protein-78-kDa. This ER-localized Hsp70 family member binds transiently to the LDLR to assist in its folding after which LDLR is released for transport to the Golgi [98, $103,104]$. There is some evidence to suggest that Grp78 may prolong its interaction with LDLR mutants to aid in folding unless it is misfolded permanently, causing the mutant LDLR to be targeted to the cytosolic $26 \mathrm{~S}$ proteasome for degradation $[98,105]$. Another chaperone, the 39-kDa-receptor-associated protein (RAP), has also been shown to facilitate folding of the LDLR $[98,106,107]$. RAP interacts specifically with nascent LDLR-like family members in the ER, blocking the binding sites of the receptors by binding the LDLA repeat of the ligand binding domain, allowing for its folding [98, 106, 107]. Willnow and colleagues demonstrated that RAP promotes the receptor folding and maturation by preventing formation of intermolecular disulfide bonds and aggregation of LDLR [107]. However, this was done in overexpression studies in human glioblastoma U87 cells. Others 
have shown that RAP-deficient mice had normal LDLR levels in the liver but lower LDLR levels in brain indicating that RAP may play a role in folding, but only in particular pathophysiological conditions [108, 109]. Even less known is the mesoderm development (MesD)'s role in the folding of the LDLR. MesD acts as a chaperone to promote plasma membrane localization of LDLR-like family members, LRP5 and LRP6 [98, 110]. In S2 insect cells expressing human LDLR, the insect homologue of MesD, Boca, facilitated the folding of LDLR and other LDLR-like proteins [111]. MesD and Boca appear to act on proteins containing EGF-like repeats and B-propeller structures indicating a wide range of specificity in their substrates of choice [98, 112].

After it is properly folded, the LDLR is transported to the Golgi complex where it matures to a $160-\mathrm{kDa}$ protein through O-glycosylation. The LDLR is then transferred to the cell surface where it will bind LDL-C via Apo-B. Here, the LDLR-LDL-C complex is internalized through clathrin-coated pits, which fuse with early endosomes. The acidic environment of the endosomes triggers a release of the complex as the LDL-C molecule is processed for degradation by the lysosome into its basic components for cell regulation/structure, and the LDLR is recycled back to the plasma membrane to wait another cycle of internalization until its degradation [4, 113].

Mutations in exons correspond to mutations in its correlative domain and hence the physiological function of the LDLR at any step in its life cycle [92, 98, 113, 114]. Over 1200 mutations in the LDLR have been identified, and they range from small missense mutations like SNPs and indels (58\%), nonsense mutations (10.4\%), and deletions/insertions (22\%) to larger rearrangements of splicing defects (9.4\%) [95]. About half of all mutant LDLR genes produce receptors with partial function due to a point 
mutation that causes a partial defect in the folding of the LDLR, its transfer to the cell surface, or its ability to bind LDL-C [95]. A patient with this kind of mutation has reduced, but not null, LDLR activity and is called receptor-defective. The other 50\% mutant of LDLR genes are true null alleles that fail to produce any detectable LDLR activity $[91,95]$. The latter is called receptor-negative. Some HoFH patients are genetic compounds with two different mutant alleles, referred to as compound heterozygotes. Receptor-deficient patients are more likely to respond to drugs like statins since there is some LDLR activity to stimulate. HoFH receptor-negative patients usually die prior to age 20 without aggressive therapy and can pass as early as 4 years $[2,15,18]$. LDLR mutations have been classified into 6 classes.

Class I mutations are described as null alleles due to the absence of protein caused by lack of processed mRNA into the secretory pathway [87, 92, 98]. Some of these null alleles produce no LDLR mRNA due to deletions in exons 1 or the promoter. However, most Class I null alleles do produce LDLR mRNA but with either rapid mRNA turnover due to non-sense mutations in exons 2 and 8 or premature termination codons due to deletions in parts of exons 13,14, or 15 [92]. Class II or transport defective alleles affect the transport of the LDLR from the ER to Golgi and will be discussed in further detail below (Figure 2). Class III mutant receptors do reach the cell surface but are unable to bind LDL-C $[92,98]$ because of mutations in the LR repeat of the ligand binding domain results in the same dysfunction as deleting the entire repeat [92]. Class IV mutations are internalization defective alleles in which LDLR will bind LDL-C but the LDLR-LDL-C complex cannot be endocytosed $[92,98]$. These rare mutations in the cytoplasmic domain called 4A or in the transmembrane domain called 4B [95], affect the ability of clathrin- 
coated pits to internalize the complex [92, 98]. Recycling deficient alleles cannot release the LDL-C ligand from LDLR and are targeted to the endosome for early degradation instead of recycling back to the plasma membrane $[92,98]$. The Class V mutations are usually the result of mutations in the EGFP domain that is responsible for acid-dependent dissociation of the receptor and ligand (Figure 2). The most recently described group of LDLR mutants, termed Class VI, contain LDLR's that mislocalize to the apical instead of the basolateral membrane in polarized cells $[98,115]$. Over $50 \%$ of mutations have been described as class II mutations, specifically class IIB, and will be focused on hereafter [92, 98]. 


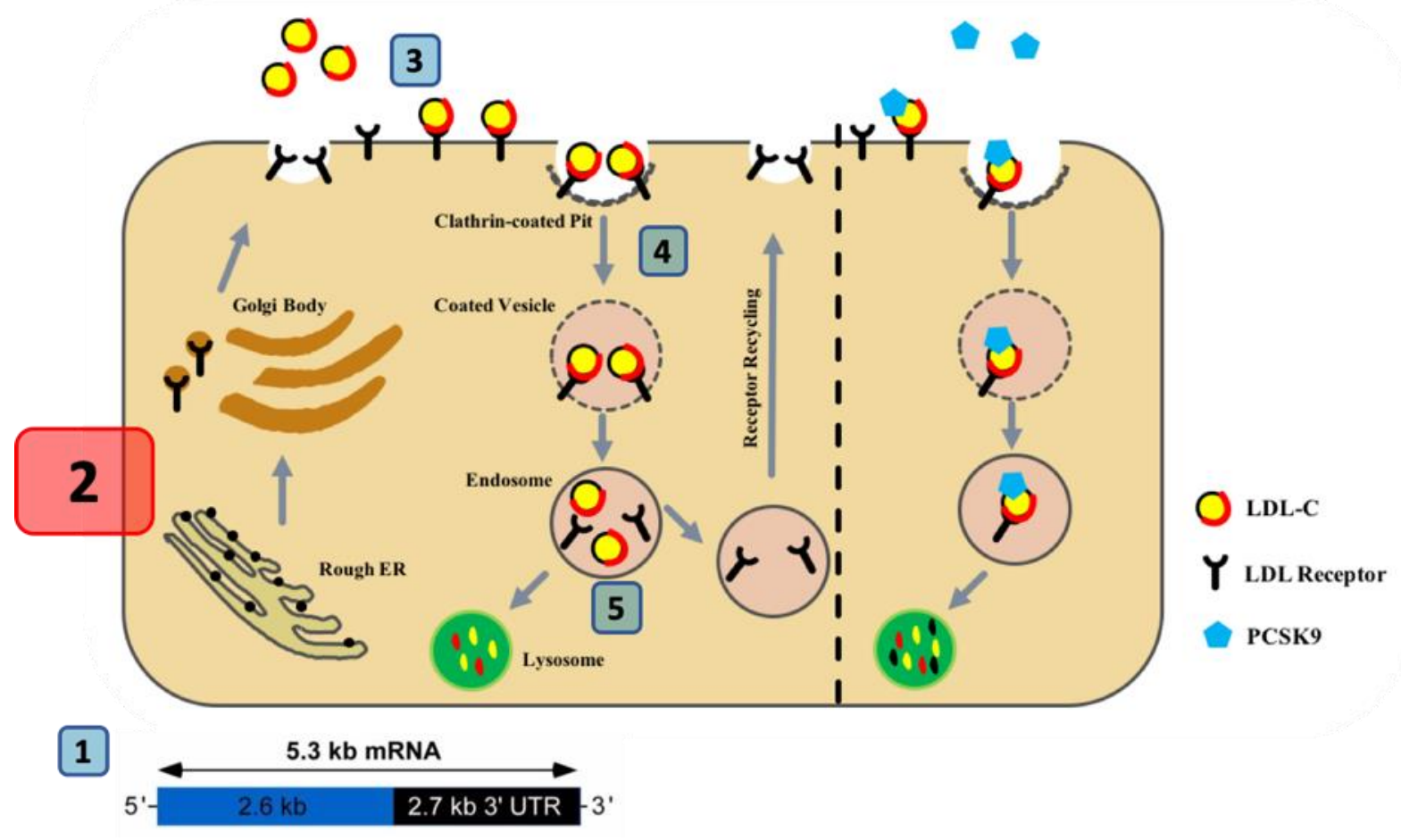

Figure 2. Schematic Representation of Human LDL-Receptor Mediated Endocytosis. LDLR is synthesized on the endoplasmic reticulum (ER) and processed into an immature $120-\mathrm{kDa}$ protein. It is then shuttled to the Golgi for further processing into a mature 160$\mathrm{kDa}$ LDLR. The mature LDLR is transported and inserted into the plasma membrane where it will bind an LDL-C molecule through apoB-100 interaction. This complex will be internalized through clathrin-coated pits and fuse with an endosome where a lower $\mathrm{pH}$ causes the release of the LDL-C from LDLR. The LDLR is recycled back to the plasma membrane to repeat this cycle approximately 100 times before it is degraded by the lysosome. There are 5 classes of mutations that affect the LDLR life cycle; 1) Lack of processed mRNA, 2) transport-defective, 3) LDL-C binding defect, 4) Clustering defect, and 5) early degradation defect. Class 2 mutations make up 50\% of FH LDLR mutations. Promoter adapted and modified from Faisal A. Al-Allaf et al. Int Arch Med. 2010; 3: 36. [90]

\section{Class II LDLR Mutation}

The normal LDLR is synthesized in the ER as a partially glycosylated precursor of $120-\mathrm{kDa}$. Upon reaching the Golgi, N-and O-linked sugars are added yielding a molecular weight of $160-\mathrm{kDa}$. Pulse chase experiments have shown this process to occur within an hour after synthesis $[116,117]$. This process is delayed or completely abolished when LDLR contains class II mutations [92]. Instead, the LDLR doesn't leave the ER [118] 
and remains at $120-\mathrm{kDa}$ until it is degraded [92, 98, 119]. Over 50\% of FH mutations identified have been classified as class II transport defective mutations. Because this mutation results in a misfolded LDLR unable to leave the ER, it is commonly referred to as a "folding or conformational disease" [98, 120, 121].

Class II defects have been clustered in the exons that encode for the ligand binding and EGFP homology domains $[92,114,117]$. Mutations affecting the cysteine-rich repeats present in both domains appear to be casually linked to the slowing or halting of the LDLR from the ER. Russell's group was the first to isolate the cDNA sequence of the 12-base pair deletion found in the $3^{\text {rd }}$ cysteine-rich repeat of the ligand-binding domain in the $\mathrm{FH}$ Watanabe rabbit [122]. This in-frame deletion eliminates 4 amino acids and hence disrupts the LDLR's movement from the ER to the Golgi. Although the deletion did not actually remove any of the cysteine residues, the change in amino acids present likely altered the folding pattern of the protein and prevented proper disulfide bond formation [122].

\section{Class IIA vs IIB}

Class II mutations can be further divided into two categories depending on the percent activity of the LDLR. Mutations that completely block receptor transport out of the ER are designated as class IIA mutations [91, 92, 114]. Class IIA mutations have been linked to mutations in the EGFP homology domain, sometimes within one of the 3 cysteine-rich repeats or within the YWTD region between repeats B and C [92]. A particular class IIA mutation, p.G565V, is completely retained in the ER as shown through co-localization western blot $[123,124]$ and has no surface LDLR or LDL internalization demonstrated by flow cytometry [123]. Jorgensen et al demonstrated two-class IIA mutant LDLRs, C646Y and W556S, were totally retained in the ER and bound to Grp78 [125]. A 
pulse-chase experiment followed by SDS-PAGE of Class IIA FH429 and FH261 fibroblasts showed zero mature LDLR compared to normal fibroblasts nearly all-mature LDLR [118].

Class IIB mutations produce LDLRs that are transported at a measurable but reduced rate $[91,92,114]$. These mutations appear to change the spacing between the highly conserved cysteine residues thereby interfering with disulfide bond formation and proper protein folding $[92,114,122]$. Studies in fibroblasts carrying Class IIB mutations showed less than $5 \%$ conversion of immature to mature LDLR [118]. This extreme case in Class IIB is due to the fact that the cell line used was from a HoFH patient. Cases of Class IIB mutations that are heterozygous do not have such a significant presence of immature

\section{LDLR.}

\section{FH Piscataway}

FH Piscataway is named from a young American HoFH patient from Piscataway, New Jersey [92]. The mutation, FH563, is localized to the $5^{\text {th }}$ repeat of the ligand-binding domain causing a 3-base pair deletion of TGG in exon 4 of the LDLR $[117,122,126]$. This removes glycine-197 and results in an in-frame mutation. When Russell's group was identifying if deletions in the cysteine-rich repeats of the LDLR would affect transport, they used both the Watanabe rabbit (12 base pair deletion) and human FH563 fibroblast cells [122].

Pulse-chase experiments with FH563 and normal fibroblasts followed by immunoprecipitation with a monoclonal LDLR antibody [122], unsurprisingly demonstrated that the normal fibroblasts' LDLR had been transported to the Golgi for processing to the mature $160-\mathrm{kDa}$ proteins within 30 minutes. 95\% of the FH563 LDLR 
remained as $120-\mathrm{kDa}$ after 2 hours. The heterozygous parents' fibroblasts were also pulsechased and showed that after 2 hours, half of their LDLR's were mature and the other half still in precursor form. This indicated FH563 inherited the same mutant allele from each parent and was homozygous for a transport-deficient receptor similar to the Watanabe rabbit [122].

FH563 fibroblasts were also carefully studied through electron microscopy to identify the distribution of the intracellular LDLR [118]. Less than 5\% of LDLR was detectable on the cell surface and in coated pits or vesicles in the endocytic pathway unlike normal fibroblast LDLR with almost all LDLR found in coated pits. Instead, most of the FH563 LDLR was present in membrane extensions of the rough ER [118]. It's hypothesized that the extensions the mutant LDLR is localized is the site where the ER is blocking exit of the LDLR and appeared to be morphologically similar to the transitional zone of the ER implicated in transport of secretory proteins to the Golgi [118, 127]. My work is focused on the FH563 mutation and its correction using CRISPR/Cas9. 


\section{PART II: GENOME EDITING AND CRISPR/Cas9}

\section{$\underline{\text { Genetic Mutations and Disease }}$}

Genetic mapping is a process that has and is used to link transmittance of a disease from parent to child through at least one gene. DNA markers isolated from both the affected and normal family members are used to pinpoint the disease-causing gene [128]. Genetic mapping has come a long way since the first mapping of a genetic disease marker (found in Huntington's disease on chromosome four) and the first comprehensive genetic map produced based on DNA sequencing with restriction enzymes. The first draft of the human genome was completed in 2003 and revealed over 20,000 genes [129]. Of those genes, 3500 have been linked to genetic diseases [130]. Monogenic diseases are usually inherited via the classical Mendelian inheritance pattern. These genetic mutations occur in less than $1 \%$ of the population. The most common contributor of genetic variation is due to singlenucleotide polymorphisms (SNPs). One nucleotide change in the 3.2-billion nucleotide pairs can lead to an amino acid conversion altering a protein's structure or function.

Understanding the causal link of a disease is necessary to create a treatment plan for the patients. Therapies vary across diseases from surgical treatment to dietary restrictions, pharmaceuticals, and gene/cell therapy. Some disorders currently have no form of treatment and require further studies to understand the disease and identify potential treatments. The development of animal models to mimic a mutant phenotype has proved beneficial in the research and clinical field in allowing translation of animal models in clinical development for patient treatments. 


\section{History of Genome Editing}

Homologous recombination (HR) is the genome's natural system to create both genetic diversity and repair DNA damage [131-133]. Capecchi and Smithies were the first investigators to demonstrate homologous recombination induction in mammalian cells between transfected plasmids [134]. Smithies' lab continued advancing HR in vitro in a substantial way: they showed that co-transfection of two non-overlapping exogenous DNA mutants into a somatic mammalian cell line would yield neomycin-resistant cells through HR [135]. Of importance, this study showed that HR increased 10-fold when a double stranded break (DSB) was introduced into one of the input plasmids. This provided the foundation for others to work at optimizing HR-DSB repair by modifying cell cycle introduction, gene homology length, introduction of double-stranded gaps, and the use of linear or circular plasmids [136-140]. This was followed with the correction of a deletion in the hypoxanthine-guanine phosphoribosyl transferase (Hprt) gene in a $\mathrm{Hprt}^{-/}$mouse embryonic stem cell (ES) line using the "pre-treated" or cut exogenous DNA [141].

A major step was taken when two labs that introduced $\mathrm{Hprt}^{-/-}$mouse ES into mouse blastocysts through microinjection produced chimeric mice [142, 143]. Further enhancing this field, Bradely's lab observed that HR using an insertion vector targeting Hprt in murine ES occurred nine times more frequently than with a replacement vector containing the same homologous sequences [144]. Site-directed mutagenesis through the combination of HDR and ES cells provided the platform for future development of mouse models to study human disease. 


\section{Gene Targeting}

Specific gene modification is referred to as gene targeting [145]. Initially, the tedious method of genetic manipulation resulted in a low number of cells containing the modification. Evidence suggested that adding DSB with an endonuclease occurred during metabolic processes such as the production of antibodies [146-150]. In 1995, Jasin et al was the first group to introduce DSBs in the genome of mouse cells. They used the yeast S. cerevisiae endonuclease, I-SceI $[151,152]$. The repair of the DSBs also required the use of donor DNA template that contained homology-flanking arms and the mutation of interest. This increased targeting efficiency 100-fold compared to no homology-flanking arms, providing further evidence that a template containing a certain length of homology on either side was necessary for successful gene targeting [153]. Although beneficial, this also limited the number of sites for modification. In addition, the DNA recognition site and cleavage function are intertwined into a domain, making it more challenging to modify the sites of recognition creating a highly inefficient system.

By contrast, zinc-finger nucleases (ZFNs) contain distinct domains for DNA recognition and nuclease activity. ZFNs were developed by combining the cleavage domain of bacterial FokI-endonuclease and two different zinc finger proteins [154-156]. Zinc fingers domains are one of the most abundant DNA binding molecules in mammals, containing 4000 binding regions within the genome [145]. A finger is made up of 30 amino acids with histidine and cysteine residues coordinating a single zinc atom within a betabeta-alpha helix structure. This finger contacts primarily 3 nucleotides and this 3 -bp target is specific to each finger. In addition, each nucleotide contacts a single amino acid side chain projecting from one end of the zinc finger alpha helical structure. This means that 
multiple zinc fingers could be combined to target multiple sites and amino-acid changes in the alpha helix altering specificity of the zinc finger. Flavobacterium okeanokoites or Fok bacteria contain an endonuclease with a nonspecific DNA-cleavage activity in its Cterminal domain. Its N-terminal domain contains a DNA binding domain. Combining the endonuclease domain of Fok with a zinc-finger protein, a chimeric restriction enzyme was created called the ZFN that will only generate a DSB when dimerized to its target site [145]. The zinc finger binds to the DNA by inserting an alpha-helix into the major groove of the DNA double helix. Each finger makes its own unique contact with the DNA due to variations in the amino acid side chains that make up the ZF. The full structure of the ZFN is N-terminal ZF DNA binding domain, a variable peptide linker, and the C-terminal FokI domain. After much optimization, it was demonstrated that inverse orientation, a six base pair space between binding sites, and no linker between the ZF and nuclease domain produced the most efficient cleavage and recombination. To promote genome editing, a pair of ZFNs must bind to a target site, one recognizing the forward strand of a target site and the other the reverse strand of the target [131]. Upon binding to their sites, the pair of Fok1 domains dimerize and cleave the DNA target, creating a DSB with a 5' overhang. This break is repaired either through non-homologous end joining (NHEJ) or homologydirected repair (HDR) [131] (Figure 3). The first mammalian cells targeted with ZFNs by Porteus and Baltimore integrated a GFP reporter into human embryonic kidney cells (HEK293) [157]. Fast forward to more recent work in modifying the human genome in CD4+ T-cells with ZFNs. Holmes group shows that ZFNs targeting X-linked severe combined immune deficiency (SCID) mutation in $I L 2 R G$ gene had the ability to modify the genetic disease mutation on both chromosomes at a high frequency in human $\mathrm{T}$ cells 
[158]. Despite ZFNs availability, it has proven difficult to engineer ZFNs to bind extended stretches of nucleotides with high affinity. An academic consortium developed an opensource library of ZFNs and protocols to help alleviate this challenge so that others may identify ZFNs that bind to desired sequences with high affinity $[131,159]$.

Transcription activator-like effector nucleases (TALENs) use an even simpler method for identifying DNA than ZFNs (Figure 3). The TAL effector domain was discovered in a plant pathogen, Xanthomonas, and consists of 10-30 tandem repeats. A single repeat domain can identify a single nucleotide by using 2 amino acid residues also called the repeat variable di-residues (RVDs) [160, 161]. Because of the simplicity in its recognition of DNA, TALEN is easily customized for its target DNA and only requires to be conjugated to the FokI nuclease domain used with ZFNs. The RVD code can be modified resulting in an increased binding affinity to target DNA sequences [131]. TALENs are designed similar to ZFNs. The TALE central DNA-binding repeat is fused to the FokI catalytic domain through a linker. It's been shown that TALENs are most functional when the spacer length is between 12-30 bp. In addition, TALENs have been shown to have fewer context-dependent effects [162]. Another plus of TALENs is the ability of the TALE repeat to be designed to target 18-bp of DNA or even longer [163]. Because of their ability to be created in a short amount of time and at a high volume, a library of TALENs targeting all of the genes in the genome has been created [131]. A disadvantage of TALENs is their cumbersome size, at least in comparison to ZFNs [131]. The cDNA encoding ZFNs is about $1 \mathrm{~kb}$ while the TALEN cDNA is $3 \mathrm{~kb}$ making the delivery more difficult. 
The main limitation of both ZFNs and TALENs are their inability to be used in high-throughput applications. Because they function through protein-DNA interactions, each target site requires engineering and cloning of a new protein [145]. In contrast, CRISPR/Cas9 is a RNA-guided nuclease whose sequence specificity is through WatsonCrick base pairing between its guide RNA and target DNA site [164].

\section{Genome editing}

ZFN

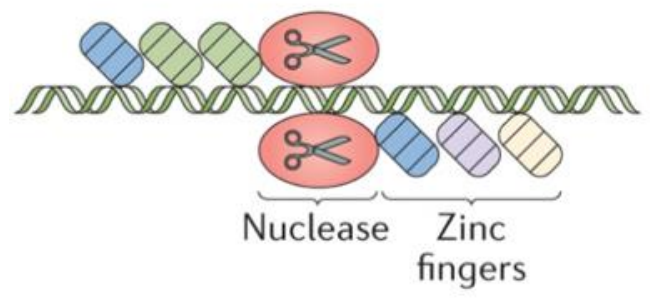

TALEN

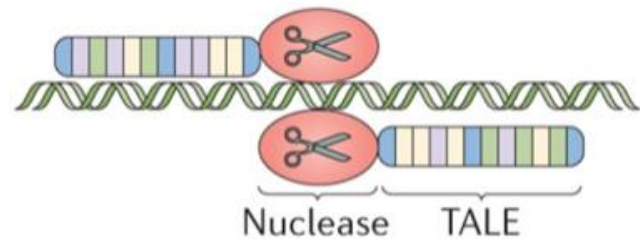

\section{CRISPR-Cas}

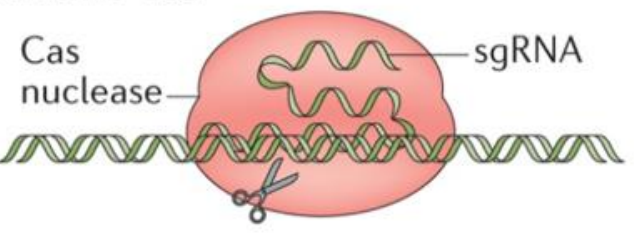

DSB repair

NHEJ

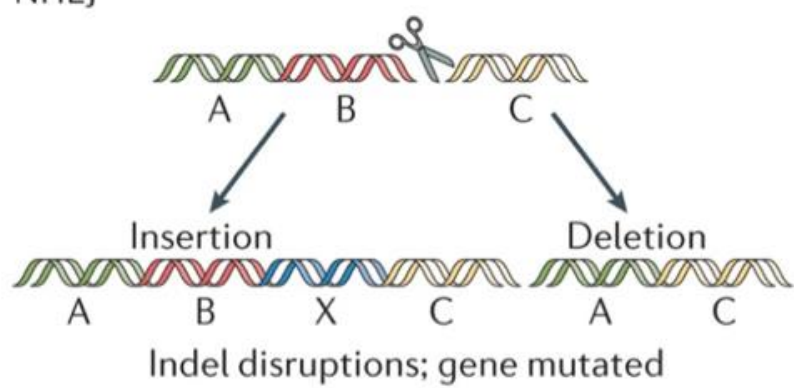

HDR

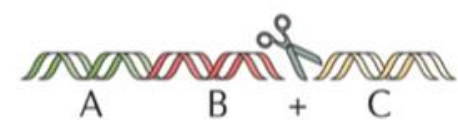

Donor template

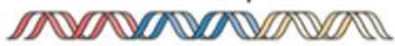

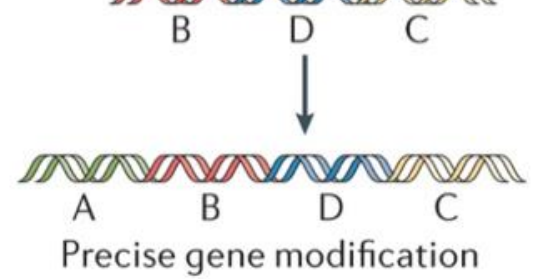

Figure 3. Representation of Tools and their Mechanisms for Genome Editing and DSB Repair. Zinc-finger nucleases (ZFNs), transcription activator-like effector nucleases (TALENs), and clustered regularly interspaced short palindromic repeats (CRISPR) and CRISPR-associated proteins (Cas) are genome modifying tools that have been utilized to induce double stranded breaks (DSBs) in DNA. ZFN and TALEN require the use of 2 DNA-recognizing proteins conjugated to a nuclease to create a DSB. CRISPR/Cas is an RNA-guided system that uses a single nuclease and guide RNA for a DSB. DSBs are repaired either through non-homologous end joining (NHEJ) or homology-directed repair (HDR). Adapted from Hao Yin et al. Nat Rev Drug Discov. 2017; 16 (6): 387-99. [165] 


\section{$\underline{\text { CRISPR }}$}

Clustered regularly interspaced short palindromic repeats (CRISPR) and CRISPRassociated proteins (Cas) are an RNA-mediated immune response mechanism used by bacteria to prevent the invasion of foreign DNA like viruses and other bacteria $[131,145$, 166]. The Cas protein can be programmed to target any site by changing its guide RNA sequence, providing a platform for high-throughput applications [164]. Before proceeding to its mechanism of action, its historical discovery deserves acknowledgement.

In the early 1990s, Dr. Francisco Mojica characterized the CRISPR locus in an archaeal microbe, Haloferax mediterranei, that grew in the extremely salty marshes off the coast of Santa Pola near the laboratory where he worked [131, 167]. Prior to this, Japanese researchers had described the presence of short direct repeats interspaced with short sequences within the genome of $E$. coli [168]. Through bioinformatics studies, the CRISPR loci would be found in 20 different microbes [169]. However, the function of these repeats was still unknown as were the "spacer sequences" between them. He used BLAST to analyze the unknown sequences. Eventually, a spacer matched the sequence of a P1 phage in an E. coli strain that was also resistant to this infectant [170]. Continuing his BLAST work on thousands of bacterial strains, of 88 with similar sequences, he found $2 / 3$ of the spacers coding for viruses or conjugative plasmids. It was concluded that the CRISPR loci encoded the instructions for an adaptive immune response that protected microbes against specific infections $[167,170]$. Another group in the French Ministry of Defense had come to a similar conclusion after finding identical tandem-repeat loci in closely related isolates from a $Y$. pestis bacterial strain from Vietnam $[167,171]$. Instead of an immune response, they claimed, "CRISPR may represent a memory of past genetic aggressions" [172]. 
The search went on to investigate if CRISPR was truly an adaptive immune mechanism in bacteria. A group of Dutch scientists working in a food laboratory at Danisco were interested in the correlation between phage resistance and the spacers in CRISPR [167]. They focused on using S. thermophilus, since it's mainly used in the dairy industry to produce yogurt and cheese. It is also phage sensitive and prone to phage attacks during yogurt making [173]. They infected the sensitive S. thermophilus until they were able to genetically isolate now phage-resistant bacteria $[167,173]$. The resistant strains acquired phage-derived sequences in their CRISPR loci and the more inserted spacers acquired, the increased resistance the bacteria demonstrated giving them immunity against subsequent bacteriophage infections [173]. In addition, they discovered that a cas 7 gene coding for Cas7 protein helped generate new spacer and repeats but not resistance. The cas 9 (originally called cas5) gene coding for Cas9 protein with two catalytic nuclease domains was needed for phage resistance $[174,175]$; hence, it was actively involved in the now confirmed CRISPR adaptive immune response.

Van der Oost's group at Wageningen University in Amsterdam took on the task of characterizing Cas proteins $[167,176]$. His group took a CRISPR deficient E. coli strain and transferred into it the CRISPR of another E. coli strain. They knocked out single genes and discovered that the whole system was essential to produce a 61-nucletoide long CRISPR RNA (crRNA) from a long precursor RNA transcribed from the CRISPR locus [176]. They performed a co-immunoprecipitation study of the Cas proteins followed by cloning and sequencing of the associated crRNA. This demonstrated that all the Cas proteins began with the 8 final bases of the repeat sequences followed by the spacer sequence and the start of the next repeat region [176]. It was necessary to verify that crRNA 
is what confers CRISPR-based resistance. For this, they created a CRISPR array to target 4 genes in the lambda phage using either anti-sense or sense sequences. The bacteria carrying this artificially designed CRISPR conferred resistance to the lambda phage, and even more so efficiently in the group carrying the sense strand [176]. This provided evidence that CRISPR could be directly programmed and led to a hypothesis of CRISPR targeting DNA [176].

Marraffini and Sontheimer at Northwestern University studied Staphylococcus's CRISPR system and noticed that the nickase gene (nes) on plasmids from the antibiotic resistant S. aureus was also present in the spacer sequence of S. epidermidis. Transfection of the $S$. aureus plasmid into the S. epidermidis bacteria was blocked and unable to be transferred. If the nes sequence was disrupted by the plasmid or spacer sequence, the plasmid was not blocked from interfering into the S. epidermidis [177]. Utilizing the nes gene in the plasmid, they inserted a self-splicing intron in the middle of the sequence. The idea was that if mRNA was targeted by CRISPR, the intronic sequence would be spliced out, and therefore, the plasmid would not affect interference. If DNA was targeted by CRISPR, the intron would prevent the spacer from matching with the plasmid DNA. It turned out that the spacer would not match the plasmid DNA and interfered with the CRISPR activity, clearly showing that DNA was targeted by CRISPR [177]. It's important to note that there is another CRISPR system that specifically targets RNA [178]. Through this discovery, they were the first to state that Cas was a programmable restriction enzyme and could potentially be used outside of bacterial systems [167].

The group at Danisco's food laboratory continued on working with CRISPR after confirming it was a bacterial adaptive immune response [173]. Their next step was to 
dissect the mechanism by which CRISPR/Cas cleaved DNA. To do this, they transfected plasmid free S. thermophilus with vector pNT1, derived from a native S. thermophilus and carries a chloramphenicol-resistance gene as its selection marker. These strains were also either cas 5 or cas 7 gene negative. Cas 5 (now known as cas 9 ) is involved in interference while $\operatorname{cas} 7$ plays a role in spacer acquisition [173]. Newly sensitive colonies were screened and from the 54, 30 were found to have acquired a new spacer-repeat in their CRISPR loci and had also lost the pNT1 vector. Interestingly, 14 different variations of spacers were found in these bacteria, all homologous to pNT1 sequences [179]. In addition, consistent with previous evidence, no chloramphenicol-sensitive colonies were isolated from the cas5 deficient bacteria that also retained their pNT1 plasmid. The cas 7 deficient bacteria did produce chloramphenicol-sensitive colonies; however, none of the colonies had acquired a new spacer in their CRISPR loci, indicating pNT1 loss was due to other circumstances. Within these two groups, they found strains with CRISPR conferred partial protection against pNT1 transformation [179]. One strain even contained a linearized pNT1 plasmid. They used this linearized plasmid to study the cutting process of CRISPR and found that it was specifically Cas5's endonuclease protospacer specific and orientation dependent activity that cleaved both bacteriophage and plasmid DNA in vitro [179]. In the presence of Cas5, the linearized plasmid contained a single blunt end cut event 3 nucleotides upstream of the protospacer adjacent motif sequence or PAM site [179]. The PAM site is a homologous spacer region in the phage genome that also must be present in the target DNA to provide immunity. The PAM site is unique to each CRISPR system in providing a sequence recognition pattern for the Cas enzymes to function [173, 180, 181]. Garneau also showed that the cuts made were in the same position relative to the PAM site regardless 
if the positive or negative DNA strand was present or if it was in early or late transcription. Altogether, this work showed that Cas9 was the nuclease cutting DNA and at specific positions encoded by the crRNAs [167].

In another collaborative effort to dissect S. pyogenes and its RNAs that could be involved in virulence control, another required component of the CRISPR system was discovered [182]. Charpentier's group used differential RNA sequencing of a class II CRISPR system. They retrieved data that small crRNAs originally from a 511-nucleotide pre-crRNA was the most abundantly expressed. These crRNAs were between 39-42 nucleotides long and low in primary transcript number indicating they likely were processed RNA. The third most expressed transcript present was small trans-activating CRISPR RNA (tracrRNA). These were different lengths of 171, 89, 75, and 65 nucleotides. The 171 and 89 nucleotide tracrRNA both contained a 25-nucleotide stretch of almost identical complementarity to the CRISPR repeats [182]. They hypothesized these differences in sizes were likely due to potential base-pairing between tracrRNA and precrRNA that lead to their co-processing and eventual generation of mature crRNA for CRISPR function. To test this, they created in frame deletions within either tracrRNA or pre-crRNA. Mature crRNA was absent in the mutant tracrRNA and the 75-nucleotide form of tracrRNA was not present in the pre-crRNA mutant. The addition of long tracrRNA restored pre-crRNA, processing in the mutant tracrRNA bacteria while an 89-nucleotide tracrRNA appeared once pre-crRNA function was restored. This indicated that non-coding RNAs, tracrRNA and pre-crRNA are required for the function of CRISPR; specifically, it is the tracrRNA that directs the maturation of pre-crRNA so that it may target DNA for cleavage by Cas enzymes [182]. Of interest, Charpentier's group also found that it was an 
RNase III-Csn1 complex that processed the tracrRNA-pre-crRNA to mature crRNA and the tracrRNA has the ability to form a stem-loop/inter-molecular RNA structure for precrRNA processing [182].

At this point, Sapranauskas in Virginijus Siksnys' lab was working on cloning the now well-known CRISPR system of $S$. thermophilus into a different bacterial genus, $E$. coli. Transfer of the system into $E$. coli provided protection against bacteriophage infection and plasmid transformation [183]. In addition, they found that the Cas9 protein was the sole endonuclease necessary for both crRNA maturation and DNA cleavage [183]. Gasiunas in Siksnys' lab continued on to study Cas9-crRNA interaction [184]. Cas9 is a large multi-domain protein with 2 catalytic domains, $\mathrm{HNH}$ and RuvC $[183,184]$. These two domains have been shown necessary for interference in vivo [183]. Although Cas9 was known to be necessary for the steps of crRNA maturation and DNA cleavage $[174,175]$, it was still unclear which motifs served which purpose. Gasiunas and his colleagues worked to isolate the Cas9-crRNA complex, using streptavidin-labeled Cas9 in S. thermophilus [184], and confirmed the presence of a 42-nucleotide crRNA that co-purified with the Cas9. Using a 55-nucleotide oligoduplex containing a PAM site with flanking identical sequences and a protospacer between them, they demonstrated that the Cas9-crRNA complex cleaved dsDNA 3-nucleotides away from the terminal end of the protospacer-adjacent to the PAM, leaving blunt ends and only in the presence of magnesium ions. This cleavage did not occur in the absence of the crRNA and a PAM site. They then went on and mutated each catalytic motif to identify each one's role in the nuclease. The RuvC mutant domain generated nicked DNA cutting the positive DNA strand (non-complementary) and the HNH mutant cleaved the negative strand only (complementary DNA), indicating each domain is active 
on opposite strands to produce a DSB. These results clearly demonstrated a mechanism for CRISPR's action [184].

Charpentier, in collaboration with Jennifer Doudna, also worked on the mechanism of CRISPR [167]. After purifying Cas9 from S. pyogenes, they tested its DNA, cleaving ability in the presence of mature crRNA, complementary to the target DNA and the absence of tracrRNA. No DNA cleavage was present until they added magnesium and tracrRNA, which pairs with the repeat sequence of crRNA and allows its maturation. They also found that the DNA cleavage occurred 3 base pairs upstream of the PAM sequence, producing a blunt cut [185]. After creating point mutations in the catalytic domains of Cas9, they demonstrated that each catalytic domain cleaves the opposite DNA strand. Using an electromobility shift assay, they also demonstrated that tracrRNA is required for target DNA recognition. Then, using different length tracrRNA lacking 5' or 3' ends and either a full-length mature crRNA or ones lacking the 5' or 3' end as well, their group found that truncated tracrRNA was still capable of supporting DNA cleavage. Truncation of the 5' end of the crRNA abolished DNA cleavage while truncation of the 3' end of crRNA still allowed DNA cleavage. This indicated to them that there must be a critical structure formed between the RNAs to allow this process to occur. Charpentier and Doudna's group describes the PAM sequence as a short sequence motif that allows the recognition of selfversus non-self in the foreign genome [185]. The PAM sequence of Type II CRISPR systems is a NGG consensus sequence. Jinek mutated the PAM site of an oligonucleotide and found that the GG was necessary for efficient DNA cleavage and either one's mutation reduced affinity of Cas9-tracrRNA-crRNA to the DNA. At this point, it had been observed that crRNA and tracrRNA functioned as individual components; however, whether they 
combined into a single unit to complete the mechanism of CRISPR was unknown. Jinek et al designed a chimeric RNA containing a target recognition sequence at the 5 ' end followed by a hairpin structure to maintain the base-pairing interaction between the tracrRNA and crRNA. Using GFP as the target gene, chimeric guide RNAs targeting GFP were able to cleave the DNA efficiently. This was a logical design of chimeric RNA that realistically could be programmed for a DNA target-binding sequence similar or more easily than ZFNs and TALENs [185].

Altering the mammalian genome using CRISPR was the next feat at task. Feng Zhang's lab at MIT proved to be the first group to engineer CRISPR/Cas systems to cleave genomic DNA in human and mouse cells [186]. Bacteria cells do not contain a nucleus so they began by combining the type II CRISPR locus from S. pyogenes Cas9 (SpCas9) with a GFP reporter and different number of nuclear localization signals to guarantee it would compartmentalize to the mammalian cell's nucleus. They transfected 293FT cells and observed that an SpCas9 attached to 2 nuclear localization signals had the highest GFP expression and hence efficiency of nucleus targeting [186]. After transfecting in the different components of CRISPR, they verified that the spCas9, in combination with a tracrRNA and pre-crRNA, were enough to facilitate efficient cleavage of the Emxl locus. Then they tested the ability to use a chimeric RNA fusion of a partial tracrRNA to a mature crRNA using a synthetic stem loop. They targeted the human PVALB and mouse Th loci and found efficient cleavage in both loci with the chimeric RNA [186]. They continued their study to explore the specificity of the chimeric RNA for genome editing. To do this, they created guide RNAs with single-nucleotide mismatches between the spacer and its mammalian protospacer target. A single-base pair mismatch from the 5' site of PAM up to 
11 base pairs away fully abrogated SpCas9 cleavage activity. On the other hand, mutations upstream in the spacer maintained cleavage of the genomic DNA against the protospacer target [186]. To further enhance the mechanism of CRISPR, Zhang's group went on to mutate the RuvC I domain within SpCas9 to convert it to a nickase (Figure 4). As a nickase, the SpCas9 should only nick the genomic DNA, which is mediated to repair through HDR rather than non-homologous end joining (NHEJ). They also introduced a homology repair template to promote the changes desired in the EMXI locus. After transfection and sequencing of the loci, a pair of inserted restriction enzyme sites found in the donor repair template were discovered and integrated in the genome demonstrating the ability of CRISPR to facilitate targeting genomic insertions [186]. This also showed that the Cas9 enzyme, as a nickase, could enable genomic editing with a decrease for off-targets [186].

After this publication, CRISPR research modifying and editing the genome skyrocketed [187-191]. It helped tremendously that publications included protocols and detailed methodology and results [166, 172, 186, 192]. In addition, many labs deposited their vectors for the various CRISPR components into Addgene repository, making it even more available for researchers. This collaboration has allowed the reproducibility of genome editing both in vitro and in vivo [187, 189-191] and the optimizing of CRISPR to work effectively and efficiently, including the use of ribosomal CRISPR and the deadCas9 [193-197]. 

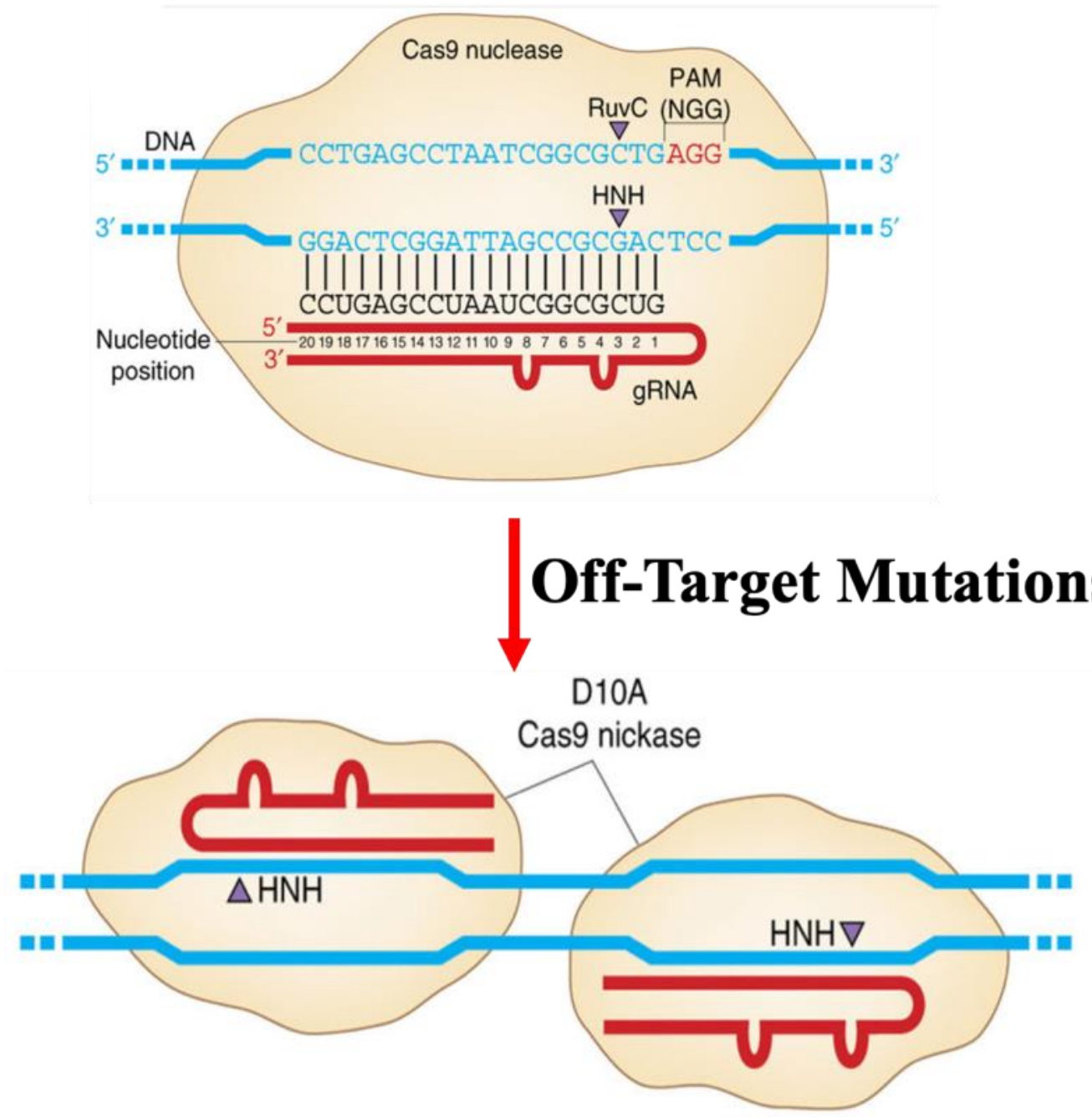

Figure 4. CRISPR/Cas9 and its Modification for Altering the Genome. The gRNA binds through complementarity to the target sequence. Because of the 2 active catalytic domains (RuvC and HNH), Cas9 enzyme creates a DSB at the 5' end of the gRNA that usually results in NHEJ for DNA repair. By inactivating one of the catalytic domains a nickase is generated that cleaves only the DNA strand that is complementary to it. Pairing 2 nickases will increase Cas9 specificity and produce two nicks each with a 5' overhang that can be repaired through HDR when a repair template is provided. Paired nickases also decrease the potential for off-target mutations. Adapted and modified from Jeffry D. Sanders et al. Nat Biotechnol. 2014; 32 (4): 347-55. [198]

There are two classes of CRISPR systems that exist across a range of bacteria and archaebacterial $[164,199]$. The components involved, and the mechanism of action determines the class. The class I system requires a large complex of multiple effector 
protein for RNA-guided target cleavage while the class II system only needs a single RNAguided endonuclease for DNA cleavage [199]. Each system is comprised of a distinctive array of direct repetitive repeats, non-coding RNAs, and a Cas gene to complete recognition and cleavage of DNA $[164,199]$. The repeats are interspaced by short variable sequences derived from the exogenous DNA targets known as protospacers. The host acquires its protospacer through recognition of fragments of invading phages or plasmids $[164,199]$. The direct repeats and protospacer is known as the CRISPR RNA (crRNA) array [164]. The crRNA is the 20-nucleotide guide sequence that will bind via WatsonCrick base-pairing to the DNA target. Adjacent to the crRNA-target must be a protospacer adjacent motif (PAM). The PAM is essential for crRNA targeting and changes for each type of CRISPR system [164]. The target sequence must be preceded by the PAM sequence, which will recognize this sequence and allow the target to try and base pair. The PAM sequence is absent in the host's genome to prevent self-cleavage [164].

The mechanism of action is uniform throughout, though the CRISPR action will be dependent on the presence and sequence of PAM [164]. The bacteria's first step is the acquisition phase in which protospacers are acquired and incorporated into the host's CRISPR locus as spacers. In the transcription phase, the CRISPR array is transcribed into pre-crRNA, which is cleaved and processed to mature crRNA by the now expressed Cas protein. Finally, in the interference stage, the Cas protein recognizes the target sequence through the guidance of the crRNA and cleaves the phage/plasmid DNA, defending itself from an infection $[164,199]$. 


\section{$\underline{\text { Current Advancements }}$}

There have been many successes and improvements with CRISPR. The most well characterized system is the type II CRISPR system. Streptococcus pyogenes (S. Pyogenes) contain the type II CRISPR that works by incorporating sequences from the invading DNA into its own genome, specifically within the CRISPR repeat sequence set up as an array within the bacterial genome [164]. Its PAM conforms to the 3'NGG consensus sequence, containing two G:C base pairs that occur one base pair downstream of the crRNA binding sequence, within the target DNA [182]. When it cleaves DNA, it creates a blunt ended cut 3 base pairs away from the PAM site [182]. It is also the most commonly used Cas (Cas9) protein for genome editing. The Cas9 has been modified to act as a nickase by mutating a nuclease domain, thereby allowing a DSB that has a higher likelihood for HDR with the addition of an oligonucleotide while decreasing off-target effects $[166,186]$. In addition, both of Cas9's catalytic domains can be mutated to act as a "dead" protein. The protein can still be guided and bind DNA, but it will not cut [197]. This allows the protein to be used to act as a transporter to deliver other factors to activate or repress the genome and study epigenetic modifications [200].

There are other type II Cas enzymes that have been discovered to mediate DNA cleavage and found to be useful in mammalian cells. Cpf1 is a class II type V CRISPR that only requires the guidance of a crRNA without a tracrRNA [201]. Although it is very similar to Cas9, its PAM sequence is 5'TNN and must be located on the 5' end of the target DNA. Its 2 catalytic residues are found in its singular RuvC domain. Unlike Cas9, if any of the catalytic residues in the RuvC domain are mutated, no cleavage activity can occur. It creates a 5-base pair- 5' overhang 18 base pairs away from the PAM site. Some 
advantages that Cpf1 may provide over Cas9 include sticky ends after cleavage, which could allow gene transfer in non-dividing cells that usually cannot be modified through HDR. Since its PAM site is 5'TNN rather than the G-rich Cas9, AT-rich regions or genomes without 3'NGG could be targeted with Cpf1 [201]. In addition, the single crRNA needed would be smaller and easier to deliver in somevectors than Cas9's about 100nucleotide tracrRNA/crRNA hybrid [201, 202]. Finally, the number of off-target effects created by Cpf1 is not much smaller than that of Cas9; however, computationally, the number of off-target sites predicted for Cpf1 is usually in the 1-12 range while Cas9 is as high as 90 sites [202, 203].

The delivery of CRISPR systems is an essential component in the success or failure of a modified genome. After identifying how many components need to be delivered (Cas, sgRNA, repair template), the delivery method can be selected. Delivery via transfection, viral infection, or even injection as protein, RNA, or DNA is suitable [204]. The expression system for efficient cleavage of the genome will be dependent on the end goal. An expression vector is appropriate for transient or stable transfection for high efficiency in a mammalian cell [204, 205]. Lentiviral transduction is the most commonly used for CRISPR genome wide screening and difficult to transfect cell lines and even in vivo [9, 204]. Adeno-associated viral (AAV) transduction is useful for transient and stable expression of only the Staphylococcus aureus Cas9 (SaCas9). An AAV is common for in vivo delivery due to its low immunogenicity and ability to preferentially infect specific tissues [33, 204]. However, it can only package up to $4.5 \mathrm{~kb}[33,204]$.

Feng Zhang's group continues to pave the way in optimizing the use of CRISPR by developing a Cas9 that would fit into an AAV. The Cas9 enzyme is large ranging in 
size from 1000-1300 amino acids [206] and in combination with the chimeric sgRNA (4.2 $\mathrm{kb}$ ) cannot be shrunk to fit into the $4.5 \mathrm{~kb}$ package. To find a Cas9 enzyme, six different sized Cas9 enzymes were transfected into 293FT cells with sgRNAs for specific genes. Of the six Cas9 orthologs tested, the Cas9 from the Staphylococcus aureus (SaCas9) had the highest cleavage activity [206]. Since another species of Cas9 was being used, various sized sgRNA also had to be tested to see if the length of the sgRNA would vary with each Cas as well. The highest editing efficiency with $\operatorname{SaCas} 9$ turned out to be 21-23 nucleotides long. In addition, using deep sequencing, their group identified a strong linear correlation between DSB scores and indel levels for each Cas9 and sgRNA pair in 293FT cells transfected with the different Cas9s and sgRNAs targeting EMX1 [206]. However, they also noticed other genes that were cleaved besides EMX1 due to homology of their sequences. These off-target sites were generated both through Watson-Crick base pairing and through guide: target heteroduplex formation [206]. The group still proceeded to target the Apob gene in vivo in the mouse genome using $\operatorname{SaCas} 9$, driven by a CMV promoter, and a sgRNA, driven by the U6 promoter, all packaged into an AAV vector. The virus was injected intravenously into C57BL/6 mice and after one week, they noticed a 5\% indel formation in the liver tissue. They extended the wait time to 4 weeks after which dissection of the liver tissue demonstrated characteristics of hepatic lipid accumulation in the Apob knockout mice with higher oil red staining [206]. They saw the opposite results when using the CRISPR system to target the Pcsk9 gene; they observed a 95\% decrease in serum PCSK9 levels and a $40 \%$ decrease in total cholesterol after just the first week of administering CRISPR [206]. Of course, it's necessary to determine if any off-target effects occurred within the genome after injection with the Pcsk9 targeting CRISPR. Deep 
sequencing of DNA extracted from the liver tissue did not reveal significant levels of indel formations; the mice did not show any signs of toxicity or any acute immune response to the virus or CRISPR [206].

Other methods of delivery of CRISPR include plasmids containing the gRNA and Cas9 mRNA, which can be delivered through electroporation or microinjection [204]. This is usually only used when generating transgenic embryos or transient expression [204]. An improvement in the delivery of CRISPR has been through the use of Cas9-gRNA ribonucleoprotein complexes (RNPs) [204, 207]. The Cas9-RNP, delivered via gold nanoparticles in mice with muscular dystrophy, had a high efficiency of gene editing in muscle tissue with low toxicity [195]. All in all, the use of CRISPR has only just begun and advancements in technology will ensure that CRISPR is utilized far beyond what we think imaginable. 


\section{PART III: THE ENDOPLASMIC RETICULUM and UNFOLDED PROTEIN RESPONSE}

\section{Endoplasmic Reticulum, Protein Folding and Chaperones}

The ER is a large and crucial component of the cell made up of a closed, yet interconnected network of tubules and flattened sacs extending from cell membrane to form a contiguous connection with the nuclear envelope. Its extensive structure is required for all the processes the ER maintains: synthesis and folding of proteins trafficking through the secretory pathway, biosynthesis of lipids and proteins, and maintaining a quality control process to prevent cell stress $[208,209]$. The ER is made up of both the smooth and rough ER. Most cell types contain a small volume of smooth ER; however, hepatocytes are rich in smooth ER [210]. It is in the smooth ER that enzymes chemically convert toxic hydrophobic compounds like pesticides to water-soluble molecules for secretion from the body [210].

The quality control system of the ER ensures that newly synthesized proteins only leave the compartment when their folding criteria have been met [208, 209, 211]. Newly synthesized unfolded proteins enter the ER. At the N-terminus of the protein is a signal sequence that is recognized by a signal recognition particle (SRP). The protein sequenceSRP complex binds to an SRP receptor in the ER membrane. This chain is then guided to the Sec61 translocon, an integral membrane protein, which acts as a tunnel to allow the translocation of the growing amino acid chain across the ER membrane and into the ER. Once fully in the ER lumen, the protein completes its folding assembly into its native structure with the assistance of ER chaperones such as Grp78 [208, 212, 213]. The protein 
can undergo further post-translational modifications in the ER though these modifications also occur in the Golgi as it does for the LDLR [98]. The properly folded protein is then released by the ER chaperone to proceed to its proper cellular compartment via vesicles [208, 213].

The specific factors and molecular mechanisms responsible for LDLR folding and maturation is still unclear. Chaperones are expressed in the ER and play a major role in the protein folding process $[105,214]$. The general chaperone, Grp78, is known to transiently bind LDLR and assist in its proper folding under normal conditions [124, 125, 215]. This correlates with studies of Grp78 acting selectively in retaining proteins in the ER [215]. Another chaperone that has been implicated in binding and facilitating the proper folding and maturation of the LDLR is the receptor-associated protein (RAP) [216]. The rate at which the ER folds proteins is dependent on the physiological and pathological reactions occurring within the biological system. The ER folding process can become susceptible to aggregation in times of inflammation, diabetes, and cancer [208].

Because the ER is responsible for processing of proteins, it requires a strict and modulated signaling mechanism to maintain homeostasis and prevent aggregation of unfolded or misfolded proteins and even correct misfolded proteins. This quality control ensures that only properly folded protein exit the ER for the Golgi, while misfolded proteins are retained in the ER. If unable to be corrected, misfolded proteins in the ER eventually undergo a process called ER-associated degradation, or ERAD. ERAD is a retrograde transport process where the misfolded protein is translocated back through the Sec61 tunnel to the cytosol. The protein is ubiquitinated and finally degraded by the $26 \mathrm{~S}$ 
proteasome [208, 217]. Otherwise, misfolded proteins can and do lead to cell death by apoptosis [213, 217, 218].

\section{Unfolded Protein Response in FH Class II Mutations}

Before some misfolded proteins can undergo the process of ERAD, the accumulation of these proteins leads to condition called "ER Stress" [213, 217, 218]. ER stress elicits a coping mechanism of "unfolded protein response (UPR)" [213, 217, 218]. The UPR's major role is to maintain protein homeostasis in the presence of accumulated un/misfolded proteins [213]. There are three known signaling pathways in mammals that are activated to adapt to the ER stress environment and "fix" the misfolded proteins [213]. The 3 major stress sensors of these pathways are IRE1 $\alpha$, PERK, and ATF6 [213] and work to restore folding capacity in the ER by controlling the expression of transcription factors and other downstream factors (Figure 5).

Inositol-requiring transmembrane kinase/endonuclease (IRE1 $\alpha$ ) is the bestcharacterized sensor of the ER stress response pathway. It's signaling is conserved across bacteria, plant, and animal $[219,220]$. This serine/threonine protein kinase and endoribonuclease catalyzes the processing of transcription factor X-Box binding protein-1 (XBP1) $[213,221,222]$ (Figure 5). The splicing of a 26-nucletoide intron from xbpl generates the transcription factor, spliced XBP1 (XBP1s) [213, 221, 223]. XBP1s regulates

UPR genes for folding, ERAD, autophagy, and organelle biogenesis [213, 224-226]. Of interest, XBP1s targets different genes in different tissues and under specific conditions for each indicating that this ER stress responder is as unique and in need of dissecting as most of the players involved in the UPR [213, 227]. 
PKR-like ER kinase (PERK) is an ER transmembrane protein whose activation through phosphorylation mediates UPR by phosphorylating initiation factor EIF2 $\alpha$ [213, 217, 228]. EIF2 $\alpha$ phosphorylation renders it inactive; this leads to an inhibition of protein translation and a decrease of misfolded proteins. Through its inactivation, EIF2 $\alpha$ also increases the translation of a transcription factor, activating transcription factor 4 (ATF4) $[213,217,228]$. It does this by recognizing an inhibitory upstream open reading frame in ATF4's 5' untranslated region [218]. ATF4 modulates another subset of UPR genes involved in antioxidant response, glutathione biosynthesis, amino acid metabolism, autophagy, and apoptosis [229-231] (Figure 5).

Activating transcription factor 6 (ATF6) is another ER transmembrane protein that mediates UPR [213]. Within the ATF6 protein structure is a cytosolic domain that renders it inactive in the ER [221, 232]. During ER stress, ATF6 translocates to the Golgi where the cytosolic domain is released by two proteases. This segment termed ATF6f (a fragment of ATF6) then goes on to act as a transcription factor to upregulate the expression of UPR genes involved in protein folding like ER chaperones and degradation like ERAD [221, 232] (Figure 5). 


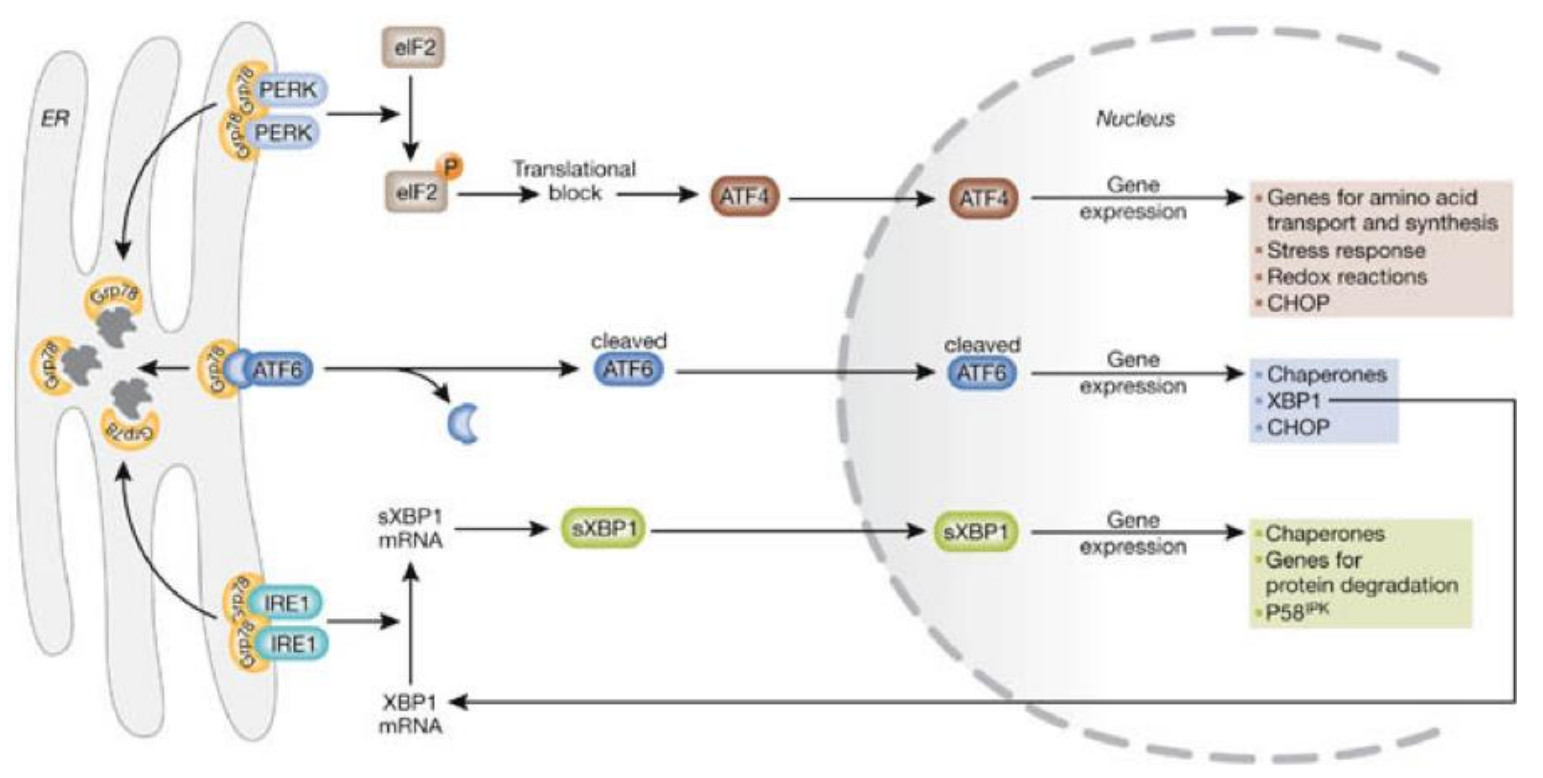

Figure 5. Schematic of the Unfolded Protein Response (UPR). When misfolded or unfolded proteins begin to aggregate in the endoplasmic reticulum (ER), Grp78 disassociates from the three ER stress receptors leading to a sequential activation of the receptors and their downstream effectors. Pancreatic ER kinase (PKR)-like ER kinase (PERK) is activated by autophosphorylation and leads to phosphorylation of eukaryotic initiation factor $2 \alpha(\mathrm{eIF} 2 \alpha)$ inhibiting translation of proteins except activating transcription 4 (ATF4). ATF4 acts on genes for amino acid synthesis and antioxidant production. Activating transcription factor 6 (ATF6) activation generates a fragmented ATF6 that acts as a transcription factor for expression of degradation and apoptotic protein CHOP. Inositol-requiring enzyme 1 (IRE1) regulates the expression of $\mathrm{X}$ box-binding protein 1 (XBP1), another transcription factor, that is spliced into its active form by IRE1. Spliced XBP1 protein (SXBP1) translocates to the nucleus regulates transcription of genes for chaperones and protein degradation. Adapted from Eva Szegezdi et al. EMBO Rep. 2006; 7: 880-85. [233]

To be brief, under stressful conditions the ER utilizes the UPR to regain homeostasis. If UPR is prolonged, severe consequences including oxidative stress, inflammation, and eventually apoptosis can occur [213, 217, 218, 229]. How does ER stress and the UPR relate to class II LDLR mutants in FH?

Evidence in the literature claims that ER stress does occur due to a class II misfolded LDLR $[124,125]$. A few studies first confirmed that class II LDLR mutants are retained in the ER [118, 124, 125]. For example, Chang cells overexpressing wild type 
LDLR or mutant W556S or C646Y LDLR were subjected to pulse chase experiments and showed wild type LDLR was processed to mature LDLR while the mutants remained in the immature form [125].

Using mass spectrometry and western blot others have demonstrated that Grp78 binds and retains class II LDLR in the ER [125]. Grp78 is an ER chaperone that binds unfolded proteins and also regulates activation of ER stress response [103, 104]. Lysates from Chang cells overexpressing class II LDLR mutants co-immunoprecipitated with Grp78 and blotted for LDLR showed binding between the two proteins. In fact, Grp78 transiently interacts with the wild type LDLR as it is processed to its mature form and its interaction is prolonged with the mutant LDLRs [125]. Another study by Sorensen and colleagues overexpressed other class IIA and class IIB mutant LDLR into Chinese hamster ovary cells (CHO) and confirmed prolonged interaction between mutant LDLR and Grp78 [124]. To test for ER stress, they looked at UPR gene and protein expression markers XBP1s and phosphorylated-PERK. Mutant G544V LDLR expressed XBP1s mRNA and had a fold induction of phosphorylated-PERK [124]. These two studies indicated that the accumulation of misfolded LDLR in the ER increased Grp78 and activated the UPR. 


\section{CHAPTER II}

\section{CRISPR CORRECTION OF A HOMOZYGOUS LDLR MUTATION IN FAMILIAL HYPERCHOLESTEROLEMIA INDUCED PLURIPOTENT STEM CELLS}

\section{$\underline{\text { INTRODUCTION }}$}

Cardiovascular disease (CVD) is the leading cause of death worldwide. While risks include lifestyle choices and environmental factors, a subgroup of the population develop early CVD due to inheritable genetic mutations. Familial hypercholesterolemia (FH) is an autosomal dominant disease primarily caused by mutations in the low-density lipoprotein receptor (LDLR) gene, resulting in supra-elevated low-density lipoprotein-cholesterol (LDL-C) that leads to premature CVD [17, 234-236]. Defective LDLR in FH presents in two forms; the more common single allele mutation, heterozygous $\mathrm{FH}(\mathrm{HetFH}), 1: 250$ [1, 237] and the rare two allele mutation form, which can be a true homozygous or compound heterozygous mutations (both referred to as homozygous (HoFH), 1:250,000) [237, 238]. Over 1200 LDLR mutations have been identified leading to a wide spectrum of disease severity depending on the mutation's effect on LDLR activity [236, 239]. While HetFH patients can generally be treated with standard hypercholesterol therapeutic approaches (i.e. lifestyle changes, statins), receptor-defective HoFH patients with $<2 \%$ LDLR activity are generally non-responsive and must rely on more extreme, costly approaches such as weekly/bi-weekly lipid apheresis [15]. Transplantation of a normal liver in HoFH patients 
has been shown to restore normal LDL-C levels, however, the lack of organ donors creates a deficit in availability

[129, 240]. In addition, the most severely affected patients can present as adolescents in their first decade of life $[17,236]$. By the time these patients are considered for liver transplant, they often have already developed severe CVD requiring a heart transplant. Therefore, mitigating the effects of genetically induced hypercholesterolemia at the earliest time possible is critical for these patients.

Because of the common donor organ shortage and challenges related to liver transplantation, alternative approaches for providing LDL-C regulation have been investigated. Hepatocyte cell therapy demonstrates efficacy in animals [41, 42, 45] and humans [51]. However, as with solid liver organs, there is a shortage of high quality hepatocytes. Since FH is a monogenic disease, it has long been the target of gene therapy which has shown promise in animal models $[41,42,45,241]$ but mixed results in humans [51]. The discovery of cellular reprogramming to generate patient specific iPSC presents the potential for generating unlimited autologous therapeutic cells [242]. The iPSC have been generated from FH patients [243-245], but the LDLR mutations are retained and require modifications for receptor-mediated LDL-C internalization [244, 245]. The genome editing system, clustered-regularly-interspaced-short-palindromic-repeats/Casassociated 9 (CRISPR/Cas9) is a relatively simple technology capable of permanent genetic modifications and has been used to repair endogenous disease-causing genetic mutations in several diseases $[187,192,246]$. Although many cell types are used for correction with CRISPR/Cas9, an advantage of correcting at the iPSC level is their 
theoretical unlimited expansion post-correction without concern for cell senescence. They also have the capability of differentiating to the cell source of choice.

As a proof-of-concept, we reprogrammed FH fibroblasts carrying a homozygous 3 bp deletion in exon 4 of the LDLR gene (GM03040). This mutation causes a Class II mutation and has $<5 \%$ normal receptor activity. This mutation is referred to as $\mathrm{FH}-$ Piscataway [92]. To introduce a permanent correction, we utilized Cas9 nickase (Cas9n) with paired single-guide RNAs (sgRNA) to generate adjacent off-set nicks in the selected genomic target. This strategy is reported to reduce off-target mutations $[186,198]$. For the repair template, we used a single-stranded oligodeoxynucleotide (ssODN) [186, 247]. After double positive transfection and magnetic sorting of the final isolated clones, we estimate an efficiency of homozygous correction of $0.2 \%$ with this approach. The HLC derived from the corrected FH-iPSC confirmed restoration of the mature LDLR protein and normalization of receptor-mediated LDL internalization compared to non-corrected FH-HLC. This demonstrates the feasibility to generate permanently restored endogenous LDLR activity in FH-HLC by CRISPR technology.

\section{MATERIALS AND METHODS}

Cell Culture. Human fetal fibroblasts (IMR90; [248]) were cultured in DMEMHG (Invitrogen, Carlsbad, CA), 2mM L-glutamine (Invitrogen, Carlsbad, CA), and 10\% fetal bovine serum (Invitrogen). Cells were maintained at $37^{\circ} \mathrm{C}$ and $5 \% \mathrm{CO}_{2}$. Cells were passaged by rinsing the cell monolayer with Dulbecco's PBS without calcium and magnesium (Invitrogen). $0.05 \%$ trypsin-EDTA (Invitrogen) was added to the cells and incubated at $37^{\circ} \mathrm{C}$ for 3 minutes. Once detached, complete media was added to cell 
suspension to inactivate trypsin. A 1:10 split ratio was dispensed into a new culture vessel. Skin fibroblasts from an FH patient (GM03040; Coriell Cell Repositories) were cultured in FH growth medium comprised of MEM (Invitrogen), 15\% FBS, 2mM L-glutamine, and $0.1 \mathrm{mM}$ Non-Essential Amino Acids (MNEAA) (Invitrogen). Cells were maintained at $37^{\circ}$ $\mathrm{C}$ and 5\% $\mathrm{CO}_{2}$. Cells were passaged as above. Reprogrammed GM3040 (3040-iPSC) were cultured on hESC-Qualified Matrigel coated plates (BD Biosciences, San Jose, CA) in mTeSR1 with media changed daily (STEMCELL Technologies, Vancouver, Canada) [245]. Cells were passaged using Gentle Cell Dissociation Buffer (GCDB; STEMCELL Technologies) with $10 \mu \mathrm{M}$ Rock inhibitor (Selleck Chemical, Houston, TX) and maintained at $37^{\circ} \mathrm{C}$ and $5 \% \mathrm{CO}_{2}$ [245]. $\mathrm{H} 1$ stem cells (WiCell, Madison, WI) were cultured in the same way as iPSC.

Genotype Analysis by Sequencing, Karyotype Analysis, and DNA Fingerprinting. The GM03040 LDLR was genotyped by Ambry Genetics (Aliso Viejo, CA) and Sanger sequencing (Genomics Core, Louisville, KY) was used to confirm the mutation using a $20 \mu \mathrm{M}$ forward primer for LDLR exon 4 (Integrated DNA Technologies (IDT); (5'-CGGCTATAGAATGGGCTGG-3')). IMR90 fibroblasts were also sequenced as a normal LDLR gene control. Corrected clones were sequenced in the Genomics Core using the same forward LDLR exon 4 primer, and the results were analyzed in Seqman Pro (DNASTAR) to determine homozygous or heterozygous correction. DNA karyotyping and fingerprinting on GM03040 fibroblasts and derived 3040-iPSC were performed by Cell Line Genetics (Madison, WI) to confirm both were karyotypically normal and preserved matching DNA fingerprints. 
Cellular Reprogramming. GM03040 fibroblasts were reprogrammed using Stemgent's MicroRNA enhanced feeder free synthetic modified mRNA reprogramming according to the manufacturer's instructions (STEMGENT, Cambridge, MA) [245].

Immunocytochemistry. 3040-iPSC were fixed with 2\% PFA/PBS (10 minutes, $24^{\circ}$ C; Electron Microscopy Sciences, Hatfield, PA), permeabilized when needed with 0.05\% Triton X-100/PBS (5 minutes, $24^{\circ} \mathrm{C}$; Sigma Aldrich), and blocked with 5\% normal goat serum/PBS (Sigma-Aldrich). A list of all antibodies used for analyses is given in Table 1. Primary antibodies were diluted in 5\% goat serum/PBS and incubated on cells overnight at $4^{\circ} \mathrm{C}$. DAPI nuclear stain (1:2000; ThermoFisher) was added (5 minutes, $24^{\circ}$ C), washed, mounted, and imaged using an Olympus IX81 fluorescence microscope (Center Valley, PA).

Teratoma Formation. 3040-iPSC were cultured in mTeSR1 on Matrigel and passaged with trypsin-EDTA five times then subcutaneously injected into NOD/SCID- $\gamma^{\text {null }}$ immunocompromised mice (Jackson Laboratory, Bar Harbor, ME) [245, 249]. Teratomas were dissected from the mice after the tumor reached $10 \mathrm{~mm}$ in diameter, followed by fixing in 4\% PFA overnight, paraffin embedded, sectioned, and stained with hematoxylin and eosin for histological examination. Tissue identification was performed on the Olympus IX81 microscope. All animal procedures were conducted in accordance with an approved University of Louisville Institutional Animal Care and Use Committee (IACUC) protocol.

Hepatocyte Differentiation. 3040-iPSC underwent directed differentiation to hepatocyte-like cells as reported previously [245, 250]. qPCR analysis for stem cell and 
hepatocyte markers (Table 2) was performed at the end of each stage (including stage 0) and immunocytochemistry at the end of stage 4.

Quantitative PCR analysis. At the end of each stage, 3040-HLC were lysed using $250 \mu \mathrm{L}$ of $0.1 \% \beta$-mercaptoethanol in RLT Buffer (Qiagen). The lysates were purified with Qiashredder and RNeasy kits (Qiagen) according to the manufacturer's instructions. RNA was quantified with a NanoDrop 1000 Spectrophotometer (Genomics Core, University of Louisville). cDNA was generated using SuperScript II Reverse Transcriptase kit (Invitrogen) and qPCR performed using PCR Supermix (Invitrogen) with primers obtained from IDT (Table 2). Amplicons were evaluated via 2\% agarose gels (Bio-Rad). $10 \mu \mathrm{L}$ of amplicons were added to $2 \mu \mathrm{L}$ TrackIt ${ }^{\mathrm{TM}}$ Cyan/Orange Loading Buffer (Invitrogen). Gels were run at $120 \mathrm{~V}$ for 45 minutes and imaged via ChemiDoc MP Imaging System (Bio$\operatorname{Rad})$.

Indocyanine-Green Assay. Indocyanine green (ICG; Cardiogreen; SigmaAldrich) was used to measure hepatocyte function. We adapted a protocol by Ho et al [251] with modifications of dissolving the ICG dry powder at $1 \mathrm{mg} / \mathrm{mL}$ in sterile water and mixing with stage 5 media at a $1: 1$ ratio to obtain a $0.5 \mathrm{mg} / \mathrm{mL}$ stock solution. Cells were imaged on the Olympus IX81 microscope at both bright field and phase settings.

Fluorescence-labeled LDL uptake analysis. Cells were plated in 8 wells of a 24well plate and taken through HLC differentiation (see above). At the end of stage 4, LDL internalization was quantified as we have previously described [245].

Western Blot. Cells were starved overnight in 5\% lipoprotein-deficient serum media (Alfa Aesar, Tewksbury, MA) augmented with $5 \mu \mathrm{M}$ Lovastatin (EMD Millipore) or excess sterols $(10 \mu \mathrm{g} / \mathrm{mL}$ cholesterol and $5 \mu \mathrm{g} / \mathrm{mL}$ 25-Hydroxycholesterol; Sigma- 
Aldrich). The following morning, the cells were thoroughly washed Dulbecco's PBS with calcium and magnesium prior to adding $200 \mu \mathrm{L}$ RIPA lysis buffer (ThermoFisher) plus protease inhibitor cocktail (ThermoFisher) to collect the cells. The DC Protein Assay (BioRad) was used for protein quantification and $20 \mu \mathrm{g} / \mathrm{sample}$ of total protein was run on a 4$10 \%$ mini-protean TGX precast gel (Bio-Rad) at 80 volts for 2 hours. Proteins were transferred onto nitrocellulose membrane (Bio-Rad) then blocked in 5\% non-fat dry milk/TBST. Membranes were incubated overnight with the LDLR antibody (1:5000; R\&D Systems, Minneapolis, MN) or Actin (1:500; Santa Cruz Biotechnology, Dallas, TX) overnight at $4{ }^{\circ} \mathrm{C}$. After 3-5 minute washes with $5 \%$ milk/TBST, the membranes were incubated in either HRP-Rabbit Anti-Goat IgG H+L (Invitrogen) or anti-mouse IgG, HRPlinked antibody (Cell Signaling, Danvers, MA) the following day for 2 hours. Supersignal West Femto Maximum Sensitivity Substrate (ThermoFisher) was used to visualize the proteins on the Bio-Rad Imager. Densitometry was performed using Bio-Rad imaging software.

\section{CRISPR Editing Design:}

sgRNA design and cloning. The MIT CRISPR Design Tool (crispr.mit.edu) was utilized to design paired guides in close proximity to the $3 \mathrm{bp}$ (TGG) deletion in exon 4 of

the LDLR gene (Chr19:11,105,170-11,105,650) (5'...TTCCACTGCCTAAGTGGCGAGTGCATCCACTCCAGCTGGCGCTGTGATGGT
GGCCCCGACTGCAAGGACAAATCTGACGAGGAAAACTGCGCTGTGGCCAC$\left.3^{\prime}\right)$. We selected paired guides 1 and 9 to use with Cas9n. The guides were cloned into pHL-H1-ccdB-mEF $\alpha$-RIH (\#60601, Addgene, Cambridge, MA) deposited by the Hotta lab [192]. Briefly, primers were designed as previously described [172]; for guide specific 
forward primers, $19 \mathrm{bp}$ (bases 2-20) of MIT sgRNA design were utilized and the first base pair was changed to a $\mathrm{G}$ to enable $\mathrm{H} 1 \mathrm{Pol}$ III transcription. Infusion homology arms (in blue) (Table 3) were added to the 5' end of each primer pair to enable cloning into the vector when cut with BamH1 and EcoRI. A PCR reaction was performed using Phusion Hot Start Polymerase (Thermo Fisher, Walton, MA) and either guide primer paired with sgUniversal Reverse primer (Table 3). The PCR product was cloned into pHL-H1-ccdBmEFa-RIH with In-fusion Cloning (Clonetech Laboratories, Inc.). Clones were sequence confirmed with our H1-Fwd primer (5'-GCATGTCGCTATGTGTTCTG-3').

Cas9n-GFP design. We obtained a humanized S. pyogenes D10A Cas9 nickase plasmid, pHL-EFla-SphcCas9(D10A)-iP-A (\#60600, Addgene), deposited by Hotta lab [192]. The puromycin gene, along with a portion of the internal ribosomal entry site (IRES), was removed by digesting with $\mathrm{KpnI}$ and BamH1. In-fusion cloning was utilized to reinstate the missing IRES sequence and concurrently insert a green fluorescent protein (GFP) gene. The primers for PCR are listed in Table 4, sequences in blue are infusion homology arms while underlined sequence is that of GFP. The Cas9n-GFP clone was sequence confirmed.

ssODN design. A 157 bp anti-sense single stranded oligodeoxynucleotide (ssODN) repair template with $50 \mathrm{bp}$ left and right homology arms were designed to insert the missing 3-nucleotides $(\underline{\mathrm{CCA}})$ while simultaneously adding silent mutations within the PAM site and guide target site $(57 \mathrm{bp}$ ) to generate a novel $\mathrm{XmnI}$ restriction site (GAATGCATTC) and prohibit further Cas9n editing by blocking guide recognition sequence (Figure 11). The oligo was obtained from IDT (Coralville, Iowa) as a 4nM ultramer oligo and was not 
HPLC purified. The ssODN sequence has been modified from chromosome 19, gene NM_00527.4 corresponding to nucleotides 750-896.

Transfection and Selection. Electroporation transfection of reprogrammed GM03040 (3040-iPSC) was modified from previously described protocols [172, 246, 252]. Cells were transfected using a square-wave electroporator, Nepa21 cell electroporator, (NepaGene, Ichikawa-City) carried out at $24^{\circ} \mathrm{C}$ at different voltages and time to determine optimum electroporation range and cell viability (Table 5). 3040-iPSC were pre-treated overnight in $10 \mu \mathrm{M}$ Y-27632 and transfected at $150 \mathrm{~V}$ for 5 milliseconds. The transfection occurred in 2-millimeter electroporation cuvettes (Bulldog Bio, Portsmouth, $\mathrm{NH}$ ) with $10^{6}$ cells in $90 \mu \mathrm{L}$ Opti-MEM and $20 \mu \mathrm{g}$ nucleic acids $(5 \mu \mathrm{g}$ Cas9n, $5 \mu \mathrm{g}$ sgRNA1, $5 \mu \mathrm{g}$ sgRNA9, and $5 \mu \mathrm{g}$ ssODN) in $10 \mu \mathrm{L}$. Transfected cells were cultured in Y-27632-mTeSR1 media. After two days, we sorted for double fluorescent positive cells via fluorescence activated cell sorting (FACS). A week prior to transfection and sorting, used media from 3040-iPSC was collected, centrifuged, filtered, and stored at $-20^{\circ} \mathrm{C}$. On the day of sorting, conditioned media was prepared by mixing used media and fresh mTeSR 1 media in a 1:1 ratio with $1 \%$ penicillin/streptomycin (Invitrogen). Basic sorting buffer was prepared using 1X DCF-PBS (ThermoFisher), 1 mM EDTA (ThermoFisher), 25 mM HEPES pH 7.0 (Invitrogen), and 1\% knock-out serum replacement (ThermoFisher) and filter sterilized. To prepare for sorting, cells were dissociated to single-cells, counted, centrifuged, and resuspended at $<10^{6}$ cells per $0.5 \mathrm{~mL}$ basic sorting buffer in a 5 -mL polypropylene tube on ice. Cells were transported to the University of Kentucky FACS facility (Lexington, KY) where cells were sorted for positive expression of both red fluorescent protein (RFP) and GFP into 35 mm dishes in conditioned:mTeSR1 plus Y-27632 media. Cells were incubated 
for 48 hours at the University of Kentucky before being transported back to our lab at the University of Louisville for continued cell culture. Percent dual positive cells was analyzed via FlowJo (Ashland, OR).

Magnetic Sorting. Transfected cells were incubated overnight in 5\% lipoprotein deficient serum media supplemented with $5 \mu \mathrm{M}$ Lovastatin. Magnetic-activated cell sorting (MACS, Miltenyi Biotec, Bergisch Gladbach, Germany) separated LDLR ${ }^{+}$3040-iPSC from LDLR ${ }^{-}$3040-iPSC. Cells were disassociated into single cells, counted, and resuspended at $<10^{7}$ cells $/ 60 \mu \mathrm{L}$ ice cold sorting buffer (DCF-PBS, $0.5 \%$ knockout serum replacement, and 2mM EDTA filter sterilized, degassed for 15 minutes by keeping the vacuum on). $20 \mu \mathrm{L}$ FcR blocking reagent (Miltenyi Biotec) and biotinylated anti-LDLRC7 antibody (1:100; Novus Biologicals, Littleton, $\mathrm{CO}$ ) were added to the cells and rocked for 2 hours at $4^{\circ} \mathrm{C}$. Cells were washed by centrifugation at $200 \mathrm{xg}$ for 4 minutes with $2 \mathrm{~mL}$ sorting buffer twice. 3040-iPSC were re-suspended in $60 \mu \mathrm{L}$ sorting buffer, $20 \mu \mathrm{L}$ FcR blocking reagent, and anti-biotin Microbeads UltraPure (1:4; Miltenyi Biotec) and incubated for 45 minutes at $4^{\circ} \mathrm{C}$ with rocking. While cells were rocking, a magnetic separation column was set up according to manufacturer's instructions (Miltenyi Biotec).

Cells were washed once more and re-suspended in $500 \mu \mathrm{L}$ sorting buffer. Magnetic separation was followed according to manufacturer and the column of $\mathrm{LDLR}^{+}$3040-iPSC was removed from the magnet and eluted with $1 \mathrm{~mL}$ sorting buffer and plunger. Cells were plated onto $35 \mathrm{~mm}$ dishes. An aliquot of the positively collected cells, negative cells, and pre-sorted cells were processed further for sorting efficiency. Cells were pipetted into a 5$\mathrm{mL}$ polypropylene tube, centrifuged, and re-suspended in supernatant left in tube after decanting ( $\sim 50 \mu \mathrm{L})$ plus $20 \mu \mathrm{L}$ FcR blocking reagent and anti-biotin-FITC antibody (1:5; 
Miltenyi Biotec). The cells were incubated at $4^{\circ} \mathrm{C}$ for 45 minutes, washed twice with $0.05 \%$ sodium azide/DCF-PBS, and re-suspended in $300 \mu \mathrm{L} 0.05 \%$ sodium azide in DCF-PBS. FACS analysis grouped cells based on FITC fluorescence.

Clonal Isolation. Clonal selection was performed based on Rodin et al [253] protocol with modifications. Dialyzed albumin was prepared as previously except using endo-toxin free bovine serum albumin (A8806; Sigma-Aldrich, St. Louis, MO). $1.6 \mu \mathrm{g} / \mathrm{mL}$ laminin-521 (ThermoFisher) and $0.2 \mu \mathrm{g} / \mathrm{mL}$ E-cadherin-Fc (\#CDH1-274H; Creative Biomart, Shirley, NY) were mixed at a 9:1 (wt/wt) ratio in di-cationic PBS and $50 \mu \mathrm{L}$ coated the inner 24-wells of Sarstedt 96-well tissue culture plates (ThermoFisher) after which they were washed twice with $80 \mu \mathrm{L}$ di-cationic PBS followed by addition of $50 \mu \mathrm{L}$ mTeSR1:albumin per well. The MACS isolated 3040-iPSCs were prepared in single-cell suspension and counted. Serial dilution of the cells to 1 cell/ $50 \mu \mathrm{L}$ was completed and 50 $\mu \mathrm{L}$ cell suspension added per laminin-521/E-cadherin-coated well. Single-celled colonies were selected and expanded into 24-well plates. Confluent clones were collected and genotyped using restriction fragment length polymorphism (RFLP).

Restriction Fragment Length Polymorphism Assay. Genomic DNA was extracted from cells following the manufacturer's protocol (Qiagen Gentra Puregene Cell Kit, Germantown, MD). PCR was performed with LDLR exon 4-F (5'CGGCTATAGAATGGGCTGGG-3') and LDLR exon 4-R (5'GAATACTTTCTTGGCATGTTGTTG-3') for 34 cycles, with denaturation at $98^{\circ} \mathrm{C}$, annealing at $62^{\circ} \mathrm{C}$, and extension at $72^{\circ} \mathrm{C} .5 \mu \mathrm{L}$ of amplicons were run on a $1 \%$ agarose gel to detect presence of PCR product. $250 \mathrm{ng}$ of PCR product, $0.3 \mu \mathrm{L} \mathrm{XmnI} \mathrm{enzyme} \mathrm{(New}$ England Biolabs (NEB), Ipswich, MA), $1.5 \mu \mathrm{L}$ 10X Cut Smart Buffer (NEB, Ipswich, MA) 
were combined with nuclease free water (Qiagen) for total volume of $15 \mu \mathrm{L}$ and incubated at $37^{\circ} \mathrm{C}$ for 2 hours. $3 \mu \mathrm{L}$ TrackIt ${ }^{\mathrm{TM}}$ Cyan/Orange Loading Buffer was added to the digested sample followed by a $2 \%$ agarose gel run at $120 \mathrm{~V}$ for 45 minutes. Heterozygous and homozygous correction was determined by observation of bands at $576 \mathrm{bp}, 384 \mathrm{bp}$, and $192 \mathrm{bp}$ on the gel relative to $1 \mathrm{kB}$ ladder (Bio-Rad, Hercules, CA). PCR products were Sanger sequenced to verify 3-nucleotide correction of the LDLR.

Off-Target Analysis. Potential off-target sites that each guide may have was listed in the MIT CRISPR Design Tool. The three highest scoring genic and three non-genic sites were selected to check for potential off-target effects of the sgRNA (Table 6). PCR primers were designed and amplified with 25 cycles, denaturation at $98^{\circ} \mathrm{C}$, annealing at $62^{\circ} \mathrm{C}$, and extension at $72{ }^{\circ} \mathrm{C} .5 \mu \mathrm{L}$ amplicons were run at $120 \mathrm{~V}$ for 30 minutes on $1 \%$ agarose gel to confirm presence of amplicon. Following this, $10 \mu \mathrm{M}$ forward primer of the selected potential off-target sequence was added to PCR product for Sanger sequencing. Analysis in SeqMan Pro (DNASTAR, Madison, WI) was performed to identify deletions, indels, and SNPs. Control non-corrected 3040-iPSC were first tested to verify sequence followed by comparison to corrected 3040-C-iPSC. 


\section{Table 1}

$\underline{\text { List of Antibodies and Fluorophores }}$

\begin{tabular}{|c|c|c|c|}
\hline Antigen & Host & Dilution & Distributor \\
\hline OCT4 & Rat & $1: 200$ & R\&D Systems, Minneapolis, MN \\
\hline SOX2 & Goat & $1: 200$ & R\&D Systems \\
\hline SSEA4 & Mouse & $1: 200$ & ThemoFisher, Walton, MA \\
\hline SSEA1 & Mouse & $1: 200$ & EMD Millipore, Billerica, MA \\
\hline TRA-1-60 & Mouse & $1: 200$ & EMD Millipore \\
\hline TRA-1-81 & Mouse & $1: 200$ & EMD Millipore \\
\hline $\begin{array}{l}\text { StainAlive }^{\mathrm{TM}} \text { TRA-1-81 } \\
\text { (DyLight }^{\text {TM }} \text { 488) }\end{array}$ & Mouse & $1: 200$ & Stemgent, Cambridge, MA \\
\hline AFP & Mouse & $1: 200$ & Invitrogen, Carlsbad, CA \\
\hline ALB & Rabbit & $1: 200$ & MP Biomedicals, Santa Ana, CA \\
\hline CYP7A1 & Rabbit & $1: 200$ & Santa Cruz Biotechnology, Dallas, TX \\
\hline CYTOKERATIN-18 & Rabbit & $1: 200$ & Santa Cruz \\
\hline LDLR & Goat & $1: 5000$ & R\&D Systems \\
\hline Actin & Mouse & $1: 1000$ & Santa Cruz Biotechnology \\
\hline Biotinylated-LDLR-C7 & Mouse & 1:100 & Novus Biologicals, Littleton, CO \\
\hline $\begin{array}{l}\text { Alexa Fluor Anti-Goat } \\
488 \mathrm{~nm}\end{array}$ & & $1: 500$ & Invitrogen \\
\hline $\begin{array}{l}\text { Alexa Fluor Anti-Goat } \\
594 \mathrm{~nm}\end{array}$ & & $1: 500$ & Invitrogen \\
\hline Goat anti-Mouse 488 & & 1:1000 & Invitrogen \\
\hline Goat anti-Rabbit 488 & & $1: 1000$ & Invitrogen \\
\hline $\begin{array}{l}\text { HRP-Rabbit Anti-Goat IgG } \\
\text { H+L }\end{array}$ & & $1: 4000$ & Invitrogen \\
\hline $\begin{array}{l}\text { Anti-Mouse IgG, HRP- } \\
\text { linked }\end{array}$ & & 1:1000 & Cell Signaling, Danvers, MA \\
\hline Anti-Biotin Microbeads & & $1: 4$ & $\begin{array}{l}\text { Miltenyi Biotec (Bergisch Gladbach, } \\
\text { Germany }\end{array}$ \\
\hline Anti-Biotin FITC & & $1: 5$ & Mitenyi Biotec \\
\hline
\end{tabular}




\section{Table 2}

List of Primers to Determine Expression of Hepatocyte Differentiation

\begin{tabular}{|c|c|c|c|c|}
\hline Gene & Primer $1\left(5^{\prime} \rightarrow 3^{\prime}\right)$ & Primer 2 (5' $\left.\rightarrow 3^{\prime}\right)$ & $\begin{array}{l}\text { Product } \\
\text { Size } \\
\text { (bp) }\end{array}$ & $\begin{array}{l}\text { Primer-BLAST } \\
\text { Annealing } \\
\text { Temperature }\end{array}$ \\
\hline POU5F1 & TGATCCTCGGACCTGGCTAA & AACCACACTCGGACCACATC & 744 & $\begin{array}{l}\text { F: } 60.03^{\circ} \mathrm{C} \\
\text { R: } 59.97^{\circ} \mathrm{C}\end{array}$ \\
\hline SOX17 & CCGCGGTATATTACTGCAACTA & CACCCAGGACAACATTTCTTTG & 371 & $\begin{array}{l}\text { F: } 58.1^{\circ} \mathrm{C} \\
\text { R: } 58.02^{\circ} \mathrm{C}\end{array}$ \\
\hline HNF4A & CCACGGGCAAACACTACGG & GGCAGGCTGCTGTCCTCAT & 250 & $\begin{array}{l}\text { F: } 61.32^{\circ} \mathrm{C} \\
\text { R: } 62.34^{\circ} \mathrm{C}\end{array}$ \\
\hline AFP & GCTGACCTGGCTACCATATTT & GGGATGCCTTCTTGCTATCTC & 384 & $\begin{array}{l}\text { F: } 57.8^{\circ} \mathrm{C} \\
\text { R: } 58.2^{\circ} \mathrm{C}\end{array}$ \\
\hline ALB & GGTGTTGATTGCCTTTGCTC & CCCTTCATCCCGAAGTTCAT & 502 & $\begin{array}{l}\text { F: } 57.93^{\circ} \mathrm{C} \\
\text { R: } 57.28^{\circ} \mathrm{C}\end{array}$ \\
\hline GAPDH & AATCCCATCACCATCTTCC & CATCACGCCACAGTTTCC & 382 & $\begin{array}{l}\text { F: } 54.14^{\circ} \mathrm{C} \\
\text { R: } 56.43^{\circ} \mathrm{C}\end{array}$ \\
\hline
\end{tabular}

\section{Table 3}

sgRNA Primers

\begin{tabular}{l|l}
\hline$\underline{\text { Guide 1 }}$ & gagaccacttggatccGGCACTCGCCACTTAGGCAGgttttagagctagaaatagca \\
\hline$\underline{\text { Guide } 9}$ & gagaccacttggatccGACTCCAGCTGGCGCTGTGAgttttagagctagaaatagca \\
\hline$\underline{\text { sgUniversal }}$ & gccegggtttgaattcaaaaaaagcaccgactcggtgccactttttcaagttgataacggactagcettattttaacttgetatttctagctc \\
\hline
\end{tabular}

\section{Table 4}

\section{$\underline{\text { Cas9n-GFP Clone Primers }}$}

\begin{tabular}{l|l|l}
\hline- & $\underline{\text { IRES in-fusion PCR }}$ & GFP in-fusion PCR \\
\hline$\underline{\text { Forward }}$ & $\underline{\text { AAGGATGCCCAGAAGGTAC }}$ & $\underline{\text { TAATATGGCCACAACgcgtatgGTGAGCAAGGGCGAGG }}$ \\
\hline$\underline{\text { Reverse }}$ & $\underline{\text { GTTGTGGCCATATTATCATCGTG }}$ & CTTAAGCCTAGGATCctaGTACAGCTCGTCCATGC \\
\hline
\end{tabular}


Table 5

Electroporation Conditions and Highlighted Optimal Condition

\begin{tabular}{|c|c|c|c|c|c|}
\hline Voltage (V) & $\begin{array}{c}\text { Length } \\
(\mathbf{m s})\end{array}$ & $\begin{array}{c}\text { Interval } \\
(\mathbf{m s})\end{array}$ & No & $\begin{array}{c}\text { Y-27632 } \\
\text { Treatment }\end{array}$ & $\begin{array}{c}\text { Electroporation } \\
\text { Survival }\end{array}$ \\
\hline 100 & 5 & 50 & 2 & Y & Y \\
\hline 125 & 2.5 & 50 & 2 & Y & Y \\
\hline 125 & 5 & 50 & 2 & Y & Y \\
\hline 150 & 5 & 50 & 2 & Y & N \\
\hline 175 & 5 & 50 & 2 & Y & N \\
\hline 200 & 5 & 50 & 2 & Y & \\
\hline
\end{tabular}

Table 6

$\underline{\text { Predicted Off-Target Cutting Sites }}$

\begin{tabular}{|c|c|c|c|c|c|}
\hline Name & Sequence & $\begin{array}{l}\text { PAM } \\
\text { Site }\end{array}$ & Mismatches & Gene & Location \\
\hline $\begin{array}{l}\text { SgRNA1 } \\
\text { OT1-1 } \\
\text { OT1-2 } \\
\text { OT1-3 } \\
\text { OT1-4 } \\
\text { OT1-5 } \\
\text { OT1-6 }\end{array}$ & $\begin{array}{l}\text { AGTGGATGCACTCGCCACTT } \\
\text { CCTGGCTGCACTCGCCACTT } \\
\text { AGTAGATGCCCTTGCCACTT } \\
\text { AGGAAATGTACTCGCCACTT } \\
\text { TCTGGATGCACTCGCAACTT } \\
\text { TGTGGATGAACTTGCCACCT } \\
\text { ATTGGATGAACTTGACACTT }\end{array}$ & $\begin{array}{l}\text { AGG } \\
\text { CGG } \\
\text { CAG } \\
\text { TGG } \\
\text { TAG } \\
\text { CAG } \\
\text { CAG }\end{array}$ & $\begin{array}{l}\text { 3MMs }[1: 2: 6] \\
\text { 3MMs }[4: 10: 13] \\
\text { 4MMs }[3: 4: 5: 9] \\
\text { 3MMs }[1: 2: 16] \\
\text { 4MMs }[1: 9: 13: 19] \\
\text { 4MMs }[2: 9: 13: 15]\end{array}$ & $\begin{array}{l}\text { NM_080740 } \\
\text { NM_173651 } \\
\text { NR_037711 }\end{array}$ & $\begin{array}{l}\text { chr19:+11105170 } \\
\text { chr3:-61568161 } \\
\text { chrX:+48796837 } \\
\text { chr2:+23697312 } \\
\text { chr22:-22874433 } \\
\text { chr2:+186667138 } \\
\text { chr17:+33434154 }\end{array}$ \\
\hline $\begin{array}{l}\text { SgRNA9 } \\
\text { OT9-1 } \\
\text { OT9-2 } \\
\text { OT9-3 } \\
\text { OT9-4 } \\
\text { OT9-5 } \\
\text { OT9-6 }\end{array}$ & $\begin{array}{l}\text { CACTCCAGCTGGCGCTGTGA } \\
\text { AACTCCAGCTGGCGCTTTGA } \\
\text { CCCACCAGTTGGCGCTGTGA } \\
\text { AATTTCAACTGGCGCTGTGA } \\
\text { CATTCCATCTGGGGCTGTGA } \\
\text { GACTGCAGGAGGCGCTGTGA } \\
\text { CCCGCCATCTAGCGCTGTGA }\end{array}$ & $\begin{array}{l}\text { TGG } \\
\text { GGG } \\
\text { AGG } \\
\text { AAG } \\
\text { GGG } \\
\text { GAG } \\
\text { GGG }\end{array}$ & $\begin{array}{l}\text { 2MMs }[1: 17] \\
\text { 3MMs }[2: 4: 9] \\
\text { 4MMs }[1: 3: 5: 8] \\
\text { 3MMs }[3: 8: 13] \\
\text { 4MMs }[1: 5: 9: 10] \\
\text { 4MMs }[2: 4: 8: 11]\end{array}$ & $\begin{array}{l}\text { NM_001198551 } \\
\text { NM_002479 } \\
\text { NM_018936 }\end{array}$ & $\begin{array}{l}\text { chr19:+11105170 } \\
\text { chr11:-32410596 } \\
\text { chr2:-239002161 } \\
\text { chr1:-96939879 } \\
\text { chrX:+62386714 } \\
\text { chr1:+203053815 } \\
\text { chr5:-140475007 }\end{array}$ \\
\hline
\end{tabular}




\section{RESULTS}

FH Fibroblasts GM03040 have a Class IIB-FH Piscataway Mutation. We acquired the FH skin fibroblasts (GM03040) from the Coriell Cell Repository (Camden, NJ) described as homozygous receptor-defective; but no genetic information was provided. We initially confirmed the inability of GM03040 to internalize cholesterol by culturing them and a normal fibroblast control (IMR90) overnight in 5\% lipoprotein deficient serum media supplemented with either Lovastatin or excess sterols, followed the next morning with incubation in low density lipoprotein labeled with 1,1'-dioctadecyl-3,3,3',3'tetramethyl-indocarbocyanine perchlorate (DiI-LDL) [245]. Lovastatin inhibits 3hydroxy-3-methylglutaryl-coenzyme A reductase (HMG-CoA reductase), blocking cholesterol synthesis and leading to an upregulation of LDLR. The excess sterols act independent of the LDLR leading to a decrease in LDLR expression. LDL internalization by the LDLR-normal IMR90 was robust in response to Lovastatin, that was abrogated in the presence of excess sterol treatment (Figure 6A). In contrast, GM03040 showed virtually no DiI-LDL internalization, even under the same Lovastatin treatment conditions that induced robust internalization in IMR90. We next wanted to know if any LDLR was produced by GM03040. Under the same conditions for the LDL internalization assay, we collected cell lysates for analysis by western blot (Figure 6B). Lovastatin treatment of IMR90 showed strong expression of the mature LDLR protein with low expression of the immature proteins. No LDLR protein was detected when IMR90 were exposed to excess sterols. In the GM03040, Lovastatin induced low level LDLR expression with the greatest increase in immature LDLR protein and very little mature LDLR (Figure 6B). Though GM03040 expresses little LDLR, even with Lovastatin treatment, excess sterols 
downregulated LDLR indicating these FH fibroblasts are physiologically responsive to cholesterol feedback control as described by Brown \& Goldstein [235]. Sequencing of the GM03040 (and IMR90 control) LDLR identified a pathological, homozygous 3-nucleotide deletion in exon 4 (c.654_656delTGG (pG219del)) (Figure 6C). This is a Class IIB mutation known as FH-Piscataway, which results in less than 5\% LDLR activity and misfolding of immature LDLR that is degraded by the proteasome pathway $[92,119]$. Together these data confirm the FH cell line contains a 3-nucleotide deletion in exon 4 of the LDLR gene resulting in little to no LDLR-mediated LDL internalization. 


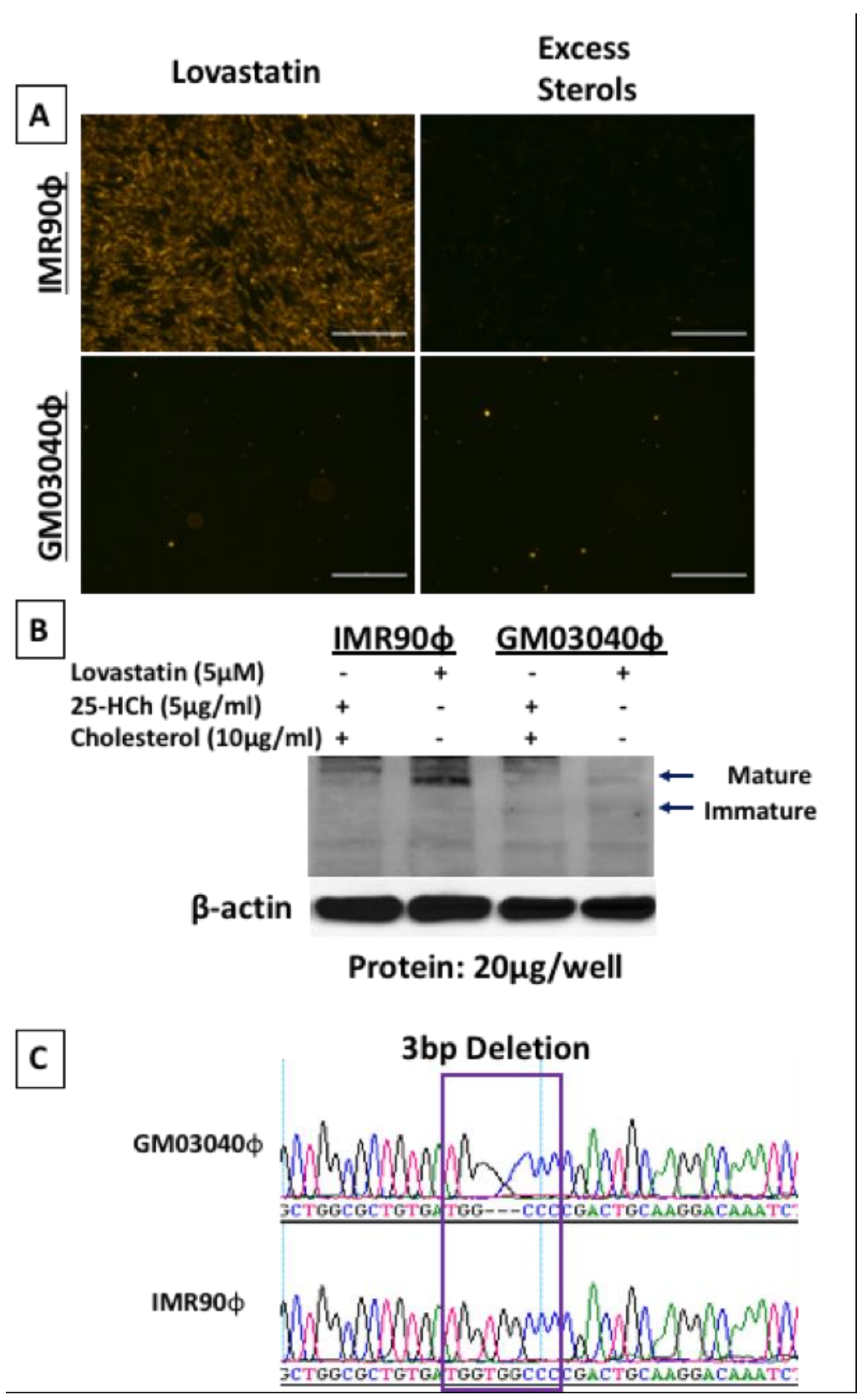

Figure 6. Mutation Identification in HoFH Fibroblasts. (A) $\mathrm{HoFH} \phi$ and control fetal IMR90 $\phi$ were treated overnight in 5\% lipoprotein deficient serum (LPDS) media supplemented with either Lovastatin or excess sterols. A significant amount of fluorescent DiI-LDL was visualized in IMR90 $\phi$ treated with Lovastatin that was abrogated with excess sterols. GM03040 $\phi$ showed impaired DiI-LDL internalization with Lovastatin treatment (Scale bars $=200 \mu \mathrm{M}$ ). (B) Western blot for LDLR shows IMR90 $\phi$ upregulate LDLR in Lovastatin and suppress LDLR when exposed to sterols. In contrast, HoFH GM03040ф express comparatively little LDLR under the same conditions. (C) Sanger sequencing revealed a homozygous 3-nucleotide deletion in exon 4 of LDLR in GM03040ф. (IMR90ф: IMR90 fibroblasts; GM03040ф: HoFH GM03040 fibroblasts). 


\section{Derivation of 3040-iPSC and Differentiation into Hepatocyte-Like Cells.}

Before reprogramming the parental GM03040, we confirmed the normal karyotype (Figure 7A) and DNA fingerprinting (not shown). We reprogrammed the fibroblasts to 3040-iPSC using a modified synthetic mRNA cocktail containing OCT4, SOX2, KLF4, CMYC, and LIN-28 [245] and began seeing colonies that were morphologically compact and refractile at approximately day 16 . Manually picking of stem cell positive expressing TRA-1- $81^{+}$colonies was initiated around day 20 for expansion into feeder-free culture (Figure 8A). 3040-iPSC demonstrated immunoreactivity with pluripotence markers TRA1-81 and UEA1 (Figure 8B), TRA-1-60, SSEA4, Oct4 and Sox2, while being negative for SSEA1 (Figure 8C). We confirmed that the reprogrammed cells were still karyotypically normal (Figure 7B) and DNA fingerprinting (not shown) established the 3040-iPSC originated from the parent GM03040 fibroblasts. Histological examination of harvested teratomas confirmed 3040-iPSC pluripotence with differentiation into derivatives of all three germ layers in vivo (Figure 8D). 


\section{A GM03040 parent fibroblast karyotype}

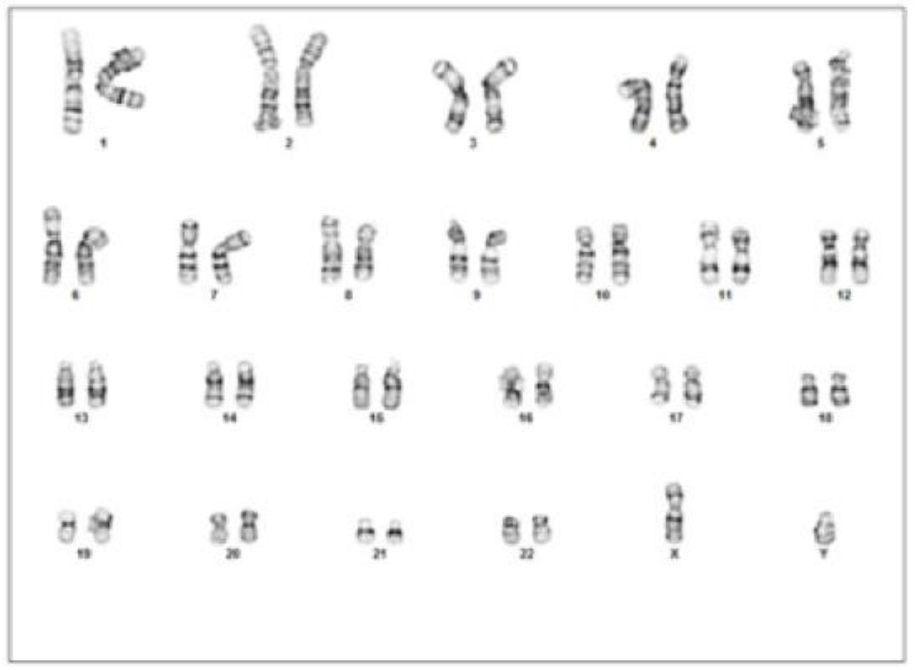

B GM03040 iPSC karyotype

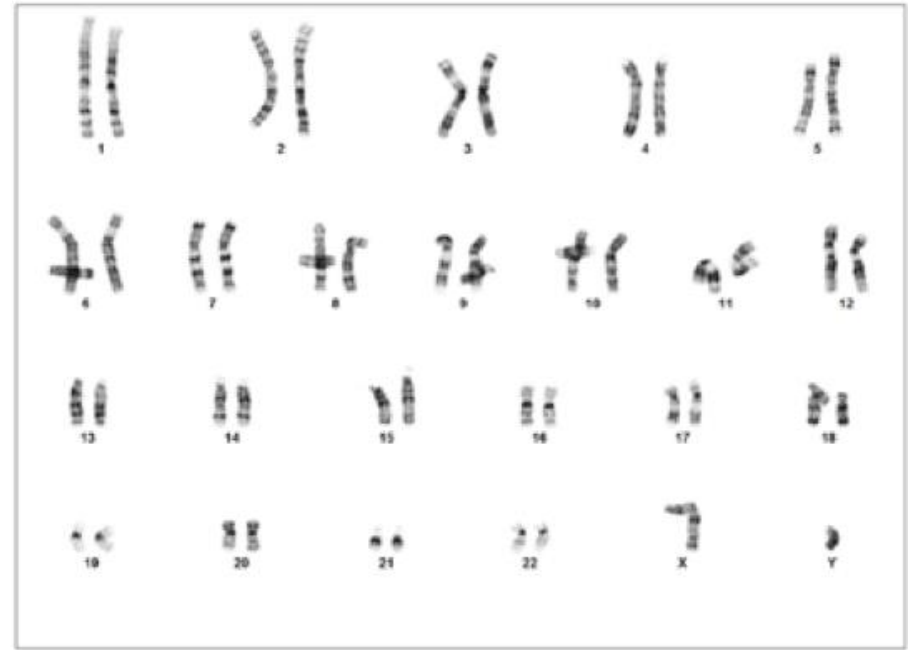

Figure 7. GM03040 are Karyotypically Normal. (A) Parent GM03040 fibroblasts and (B) reprogrammed 3040-iPSC have a normal chromosomal arrangement as seen by karyotyping. 


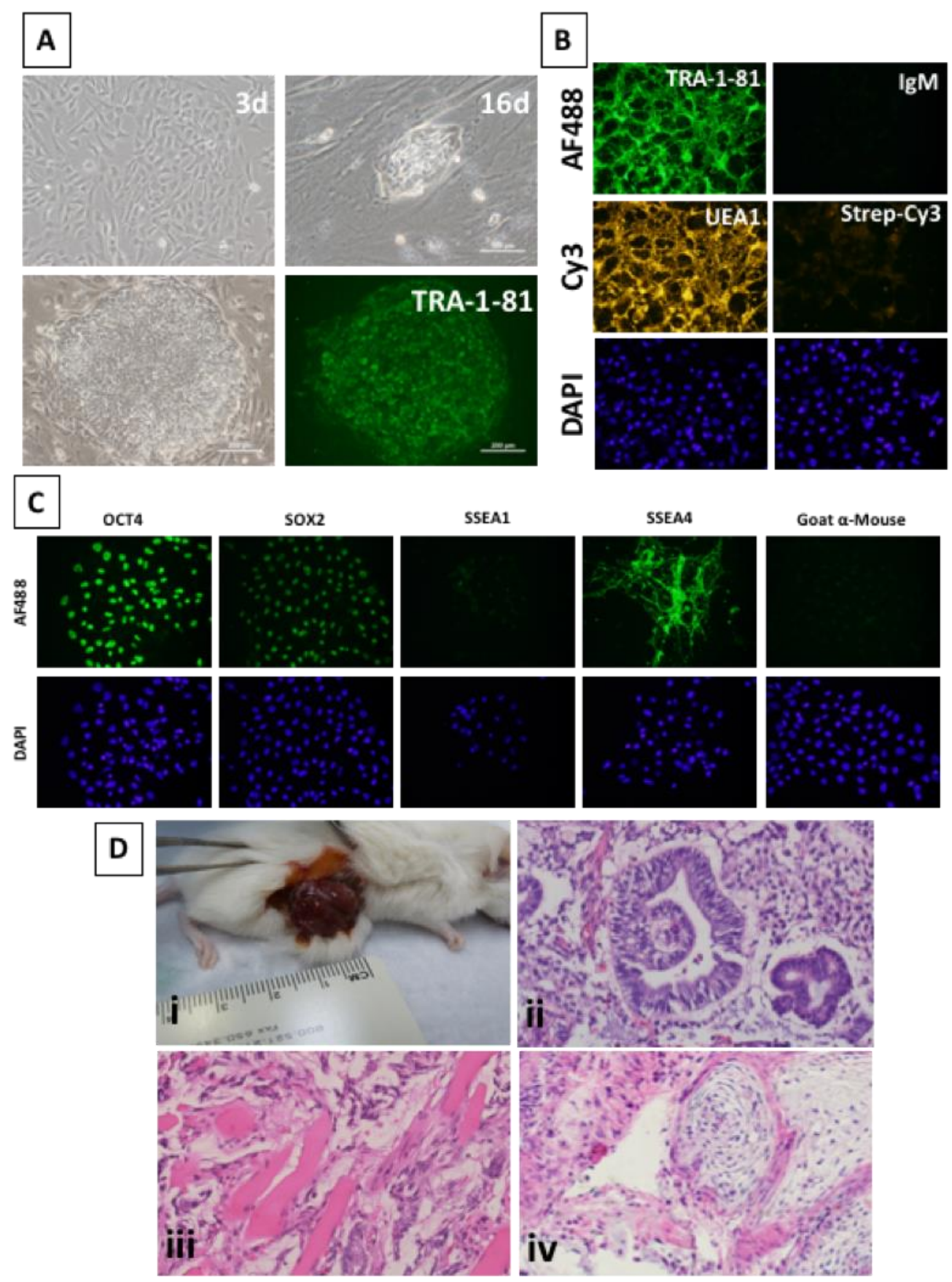

Figure 8. Reprogrammed 3040-iPSC are Pluripotent. (A) An iPSC colony is visible post reprogramming, characterized by its small, clustered appearance at Day 16 (Scale bar = $100 \mu \mathrm{m}$ ) and live staining with pluripotent marker TRA-1-81 at Day 18 (Scale bars $=200$ $\mu \mathrm{m})$. (B) Assessment of expanding 3040-iPSC pluripotence is seen with positive TRA-181 and UEA1 immunostaining. (C) Micrographs further confirmed expression of pluripotent markers TRA-1-60, SSEA4, Oct4 and Sox2, while being negative for mouse SSEA1. (D) Teratoma generated in immunocompromised mice from 3040-iPSC (i) differentiated into the 3 germ layers: endoderm-respiratory epithelium (ii), mesodermmuscle (iii), and ectoderm-nerve ending (iv). 
Most LDLRs are found on liver hepatocytes and are responsible for most LDL regulation [235]; therefore, we wanted to confirm 3040-iPSC capability to differentiate toward hepatocyte-like cells (HLC). Following a previously described five stage protocol [245, 250], 3040-iPSC showed expression of stage specific markers after completion of each differentiation stage as demonstrated by qPCR (Figure 9A; Table 2). As we have shown previously [245], 3040-iPSC undergo differentiation, as demonstrated by progression of expression of pluripotence marker octamer-binding transcription factor 4 (OCT4) at stage 0, definitive endoderm marker sex determining region Y box 17 (SOX17) [250], in stage 1, hepatic lineage specification as indicated by expression of hepatocyte nuclear factor 4a (HNF4A) in stages 2-5, the early hepatocyte marker alpha fetal protein (AFP) [250] beginning in Stage 2, and standard albumin (ALB) [250] expression initiated during stage 3. To examine protein expression, we differentiated 3040-iPSC to the end of stage 4 and used immunocytochemistry (ICC) for detection of commonly associated hepatocyte markers, albumin, AFP, CYP7A1, and cytokeratin 18 (Figure 9B) [245, 250]. Finally, we tested 3040-HLC functionality via an indocyanine green (ICG) exclusion assay, demonstrating 3040-HLC could take up and clear the green dye within 24 hours (Figure 9C). These data confirm that the reprogrammed GM03040 FH fibroblasts are pluripotent and can specifically differentiate to functioning HLC. 


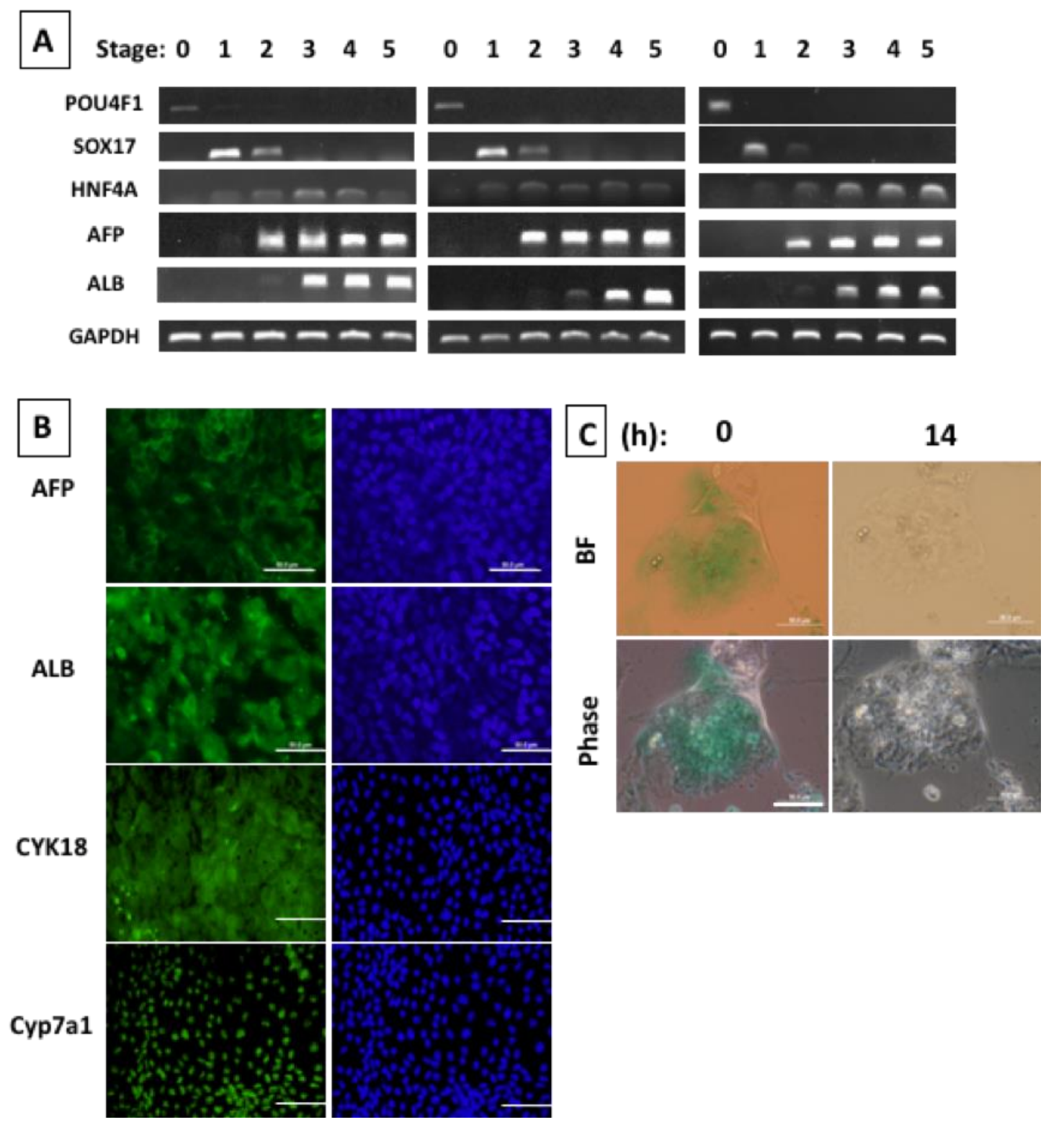

Figure 9. Hepatocyte-Like Cells (HLC) can be Derived from 3040-iPSC. (A) Stagespecific markers were tested at both mRNA expression level (POU4F1, SOX17, HNF4A, AFP, and ALB) by PCR (n=3) and (B) protein level (AFP, ALB, CYK18) by immunocytochemistry $(\mathrm{n}=2)$ in 3040-HLC (Scale bars $=50 \mu \mathrm{m})$. (C) HLC function was tested by uptake and exclusion of indocyanine green dye (ICG) over $24 \mathrm{~h}$ (Scale bars $=50$ $\mu \mathrm{m} ; \mathrm{BF}=$ bright field). 


\section{CRISPR Correction of Homozygous 3-nucleotide Deletion in the LDLR in}

3040-iPSC. Our lab has previously restored physiological function of the LDLR in FHiPSC using an episomal transgene; however, plasmid retention is compromised without continuous antibiotic selection [245]. We opted to use CRISPR/Cas9 to permanently correct the 3 bp pathogenic deletion in the 3040-iPSC. A schematic outline of our genome editing approach is presented in Figure 10. Using the MIT CRISPR Design Tool (crispr.mit.edu), we input the exon 4 LDLR sequence of the FH patient for identification of sgRNA. Thirteen possible sgRNA were found that could be used with wildtype Cas9 that were arranged in suggested pairs for use with Cas9n. We utilized the Cas9n with a pair of offset sgRNA since this grouping had previously been successful in facilitating specific genome editing in human cells [246]. The double offset nicking approach has also shown a higher likelihood of forcing HDR to improve specific repair and reduce off-target mutations. Our guide pairs (sgRNA1 and sgRNA9) were selected based on Ran et al criteria [246, 252]. The guides generate 5 ' overhangs with a +2 offset and create at least one nick within 6 bp of the target mutation site to increase the probability of HDR [186, 246]. HDR requires a repair template for the DNA to utilize and precisely modify the genome [186, 198, 246, 247]. We designed an anti-sense ssODN template with right and left $50 \mathrm{bp}$ homology arms flanking the deletion in exon 4 of LDLR. The repair template contained the deleted 3-nucleotides for inserting the missing $3 \mathrm{bp}$ (ACC) and silent mutations in the protospacer adjacent motif (PAM) of sgRNA 1 within the first $10 \mathrm{bp}$ upstream of the PAM site in sgRNA 9 to minimize rebinding after genome repair and to introduce a novel XmnI restriction site (Figure 11A,B) [186, 198, 246, 247]. 


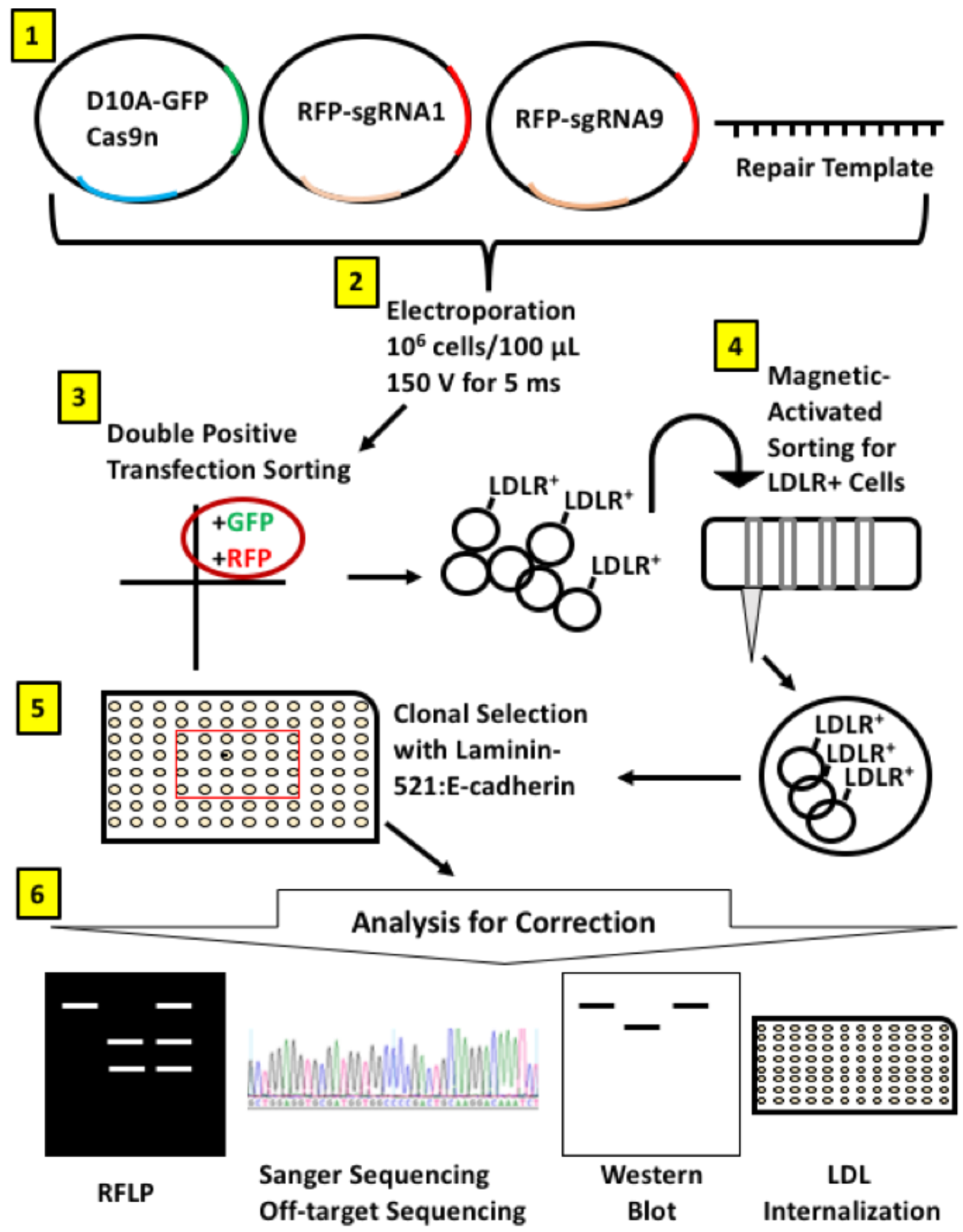

Figure 10. CRISPR Correction Strategy Methodology Schematic. (1) 3040-iPSC were transfected with $5 \mu \mathrm{g}$ Cas9n, $5 \mu \mathrm{g}$ sgRNA1, $5 \mu \mathrm{g}$ sgRNA9, and $5 \mu \mathrm{g}$ ssODN using (2) a NEPA21 square-wave electroporator. (3) Double positive $\left(\mathrm{GFP}^{+} / \mathrm{RFP}^{+}\right)$cells were selected for expansion via fluorescence sorting. (4) LDLR+ cells were enriched by magnetic sorting and expanded in culture. (5) Clones were isolated on laminin-521 + E-cadherin-Fc substrate then (6) analyzed for correction of the LDLR via RFLP, sequencing, western blot, and LDL internalization. 


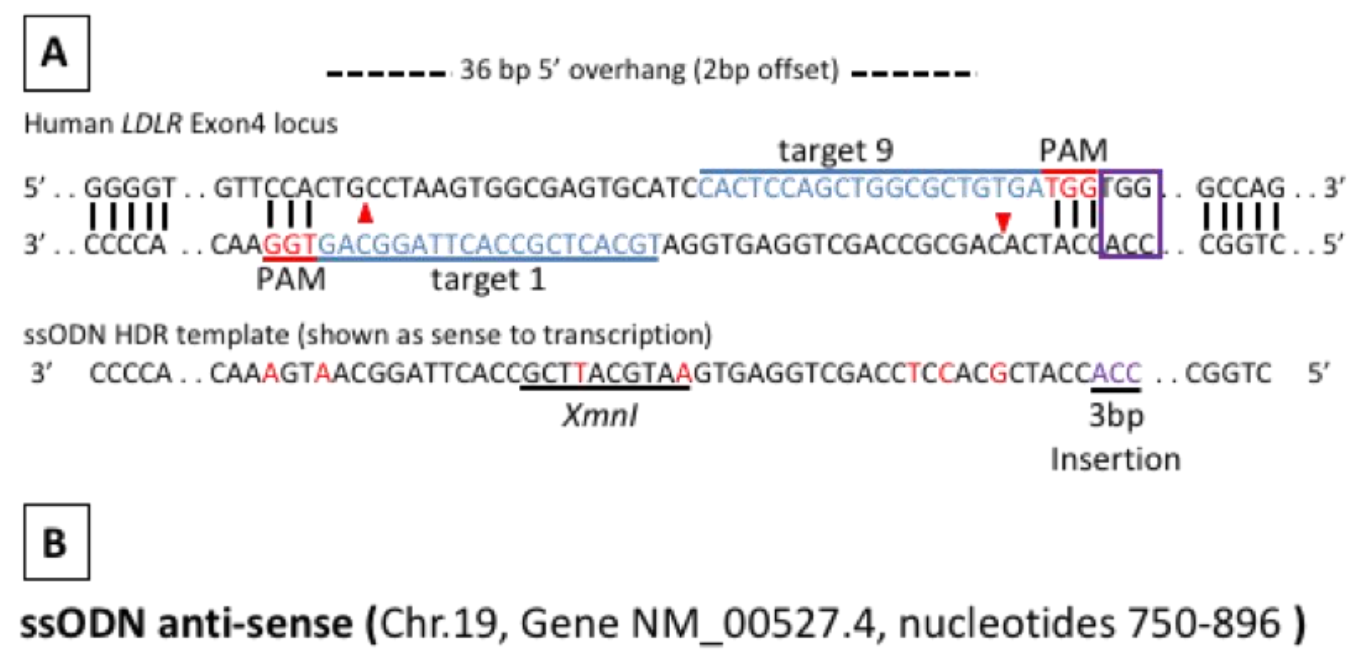

5'-CTGGCCCCGCCCATACCGCAGTTTTCCTCGTCAGATTTGTCCTTGCAG

TCggggCCAccatcgcacctccagctggagtgaatgcattcgccacttaggcaatgaaaCTC

GAAGGCCGAGCAGGGGCTACTGTCCCCTTGGAACACGTAAAGACCCC-3'

Homology arms

Silent mutations

3bp insertion

-Xmnl site

Figure 11. CRISPR ssODN Design. (A) Diagram of the CRISPR/Cas9 design to target the mutation site of the LDLR. Paired single-guide RNA (target 1 and target 9) were selected to target the mutation site, and a single-stranded oligodeoxynucleotide (ssODN) template was created to mediate HDR. The ssODN contains a 10-nucleotide sequence (underlined) as a novel XmnI restriction enzyme site, additional silent mutations (red nucleotides) at the PAM for sgRNA 1 or within 10 bp upstream of the PAM for sgRNA 9 to prevent re-binding of guides and cleavage with Cas9n following repair, and the 3 bp insertion (purple). (B) $157 \mathrm{bp}$ single-stranded oligodeoxynucleotides (ssODN) repair template sequence. $50 \mathrm{bp}$ left and right homology arms (blue) surround the sequence target (57 bp). Silent mutations (red) were inserted to prevent further re-binding of Cas9n post-correction and create a novel XmnI site (underlined). $3 \mathrm{bp}$ (CCA) insertion (purple) for correction was built into the sequence.

We obtained Cas9n and sgRNA plasmids (Addgene \# 60600 and 60601, respectively) deposited by the Hotta lab [192] and cloned in our selected sgRNA (Figure 10, Step 1). The sgRNA plasmid contains a red fluorescent reporter (RFP) that can be used 
to select for cells positively transfected with the sgRNA. We opted to use double fluorescence cell sorting post-transfection rather than antibiotic selection to potentially maximize selection of transfected cells. The Cas9n plasmid deposited by Hotta was modified to incorporate GFP into the vector. Before transfection, we optimized electroporation voltage necessary for highest transfection efficiency, referencing NEPA21 electroporator conditions by the Hotta lab [192] (Table 5). We then transfected the 3040iPSC with $20 \mu \mathrm{g}$ total nucleic acids $(5 \mu \mathrm{g}$ Cas9n, $5 \mu \mathrm{g}$ sgRNA1, $5 \mu \mathrm{g}$ sgRNA9, and $5 \mu \mathrm{g}$ ssODN) (Figure 10, Step 2). After 48 hours, we sorted for dual positive (i.e. GFP \& RFP) cells to subculture only cells transfected with both Cas9n and guide plasmids (Figure 10, Step 3). We collected $2.4 \%$ of the $13.1 \%$ population expressing both RFP and GFP (Figure 12A). Because we used 2 plasmids for each guide, we could not guarantee that sorted cells contained both guide plasmids for correction. To enrich the population of double-positive transfected cells that actually contained the $3 \mathrm{bp}$ insertion, we treated the culture with Lovastatin to upregulate LDLR expression and targeted the cell surface receptor for magnetic-activated cell sorting (MACS) (Figure 10, Step 4). Using a biotinylated-C7LDLR antibody, we enriched for $\mathrm{LDLR}^{+}$cells within the bulk of transfected cells. We analyzed the percentage of $\mathrm{LDLR}^{+}$cells collected (green dots) after MACS by FACS. FACS analysis showed a mix of $\operatorname{LDLR}^{+}$(green dots) and $\mathrm{LDLR}^{-}$(red dots) before sorting (left box). After MACS, we confirmed a collection of $19.1 \% \mathrm{LDLR}^{+}$cells, or corrected cells, with a clear shift of green dots to the right (Figure 12B). After expanding the bulk $\mathrm{LDLR}^{+}$cells we adopted the clonal isolation protocol developed by the Tryggvason lab utilizing laminin-521 and E-cadherin-Fc substrates in mTeSR1-albumin media (Figure 10, Step 5) [253]. Using a clonal dilution approach, we plated 120 cells and after 7 days of 
culture, identified 16 wells with single colonies (empty and multi-colony wells were counted as null clones) for a cloning efficiency of $13 \%$. The clonal colonies were expanded for analysis and cryopreservation.

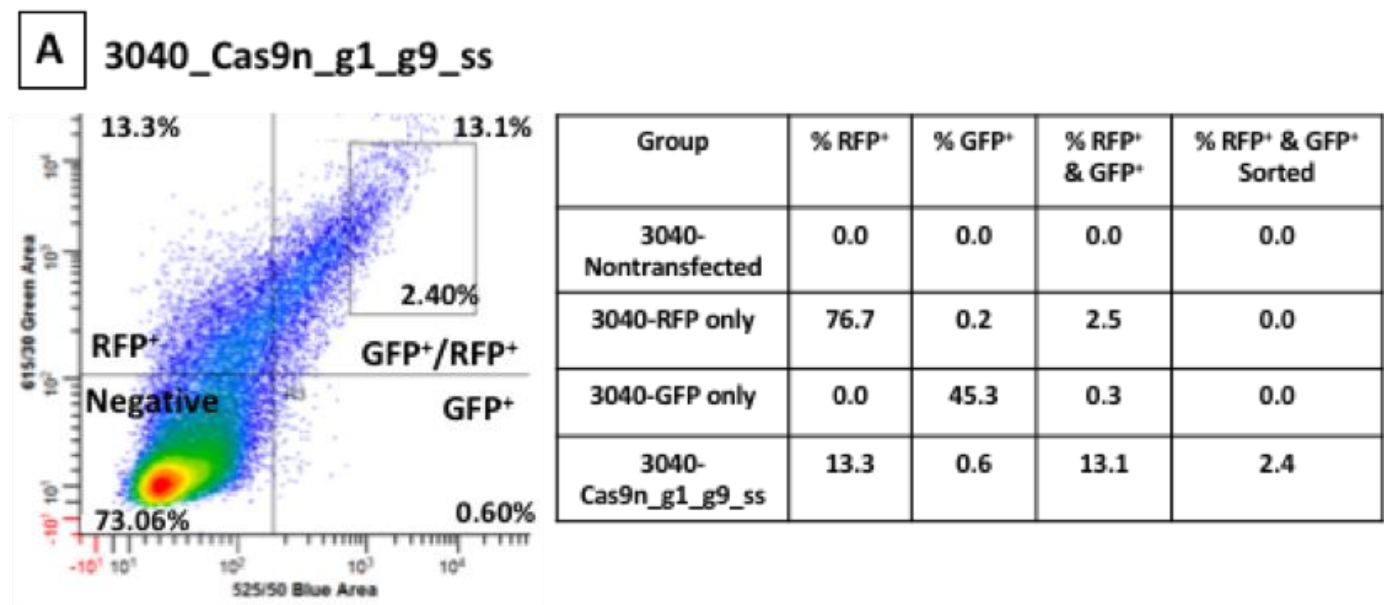

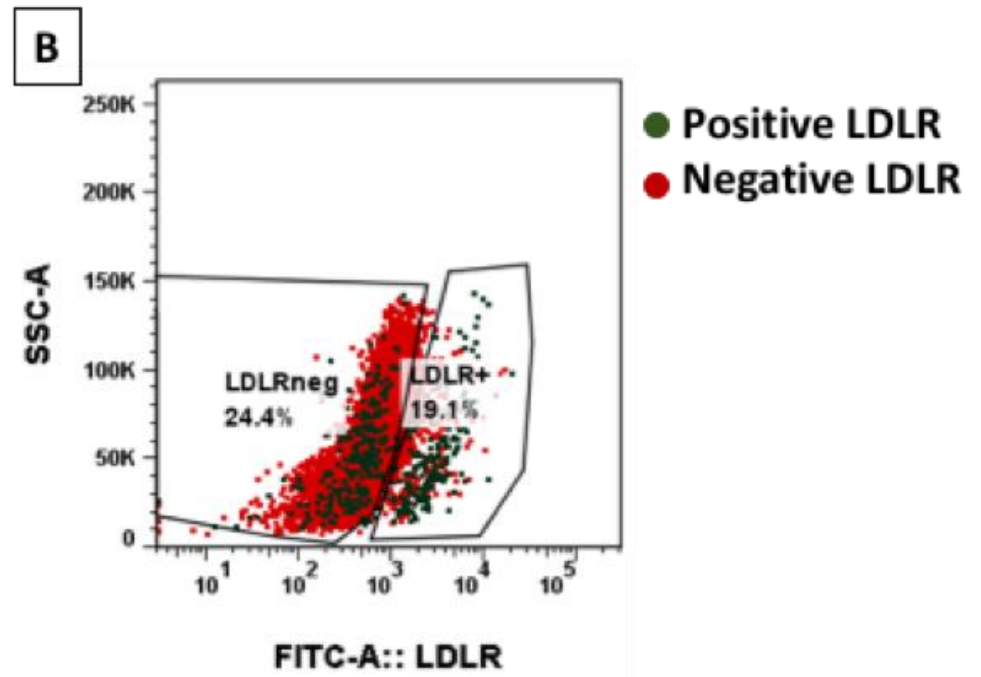

Figure 12. CRISPR Positive Sorting. (A) After transfection, 3040-iPSC were sorted for double positive RFP and GFP cells via FACS. $13.1 \%$ of cells were dual positive and $2.4 \%$ of the population was collected and expanded to increase specificity of positively transfected cells. (B) Magnetic-activated cell sorting (MACS) was performed on the FACS sorted 3040-iPSC to enrich for $\mathrm{LDLR}^{+}$expressing cells. Pre-MACS sorted cells had a mix of both positive (green dots) and negative (red dots) $\mathrm{LDLR}^{+}$cells. After MACS sorting, a clear shift to the right showed $19.1 \% \mathrm{LDLR}^{+}$cells were collected. 
Since we created a donor repair template that contains a unique XmnI restriction site (Figure 11), we could use restriction fragment length polymorphism (RFLP) analysis to check for potential correction of the 3040-iPSC clones. Additionally, we could see if it was a homozygous or heterozygous clone by band size (Figure 10, Step 6). The expected LDLR PCR product without any enzyme or correction is 576 bp as indicated by 3040 -iPSC non-transfected control (Figure 13A). A homozygous corrected clone would have two bands at 384 bp and $192 \mathrm{bp}$ while a heterozygous clone would also retain the $576 \mathrm{bp}$ band. RFLP of the first clone (3040-C) tested indicated homozygous correction by the presence of only 2 bands of the correct size (Figure 13A). RFLP analysis of the other clones showed an additional 9 homozygous corrections and 6 heterozygous corrections (Figure 13B). To confirm the RFLP results, we performed Sanger sequencing on all PCR positive clones. Four clones were found to be homozygous corrected by sequencing; however, RFLP demonstrated retention of the $576 \mathrm{bp}$ band (Clone 2, 3, 4, 11). We presumed these were not clonal and removed them from consideration. For 12 clones, the RFLP and Sanger sequencing results agreed. Interestingly, sequencing indicated that not only did the heterozygous corrected clones (C12 and C14) only correct a single allele, but they also obtained different indels in the non-corrected allele proximal to sgRNA1's target sequence. In addition, our selected clone (3040-C) was determined to be heterozygous in 2 silent mutation points denoted by the green box (Figure 13C). From these successfully isolated clonal colonies, our efficiency of homozygous correction from the final isolation of 10 (from 12) homozygous corrected clones is $83 \%$. Since sgRNA can target multiple regions within the genome [198], we analyzed for off-target mutations. We selected 3 non-genic and 3 genic regions identified by the CRISPR Design Tool for both sgRNA1 and sgRNA9 
with the highest potential for off-targeting (Table 6). Sequencing of 3040-C compared to non-corrected 3040-iPSC indicated no changes in the selected regions of the genome (not shown). While we were fortuitous in not detecting off-targets within the highest selected regions, this may not be the case with other targeted mutations. These results illustrate that the 3-nucleotide deletion was homozygous corrected without additional mutations in the homozygous FH-iPSC. 

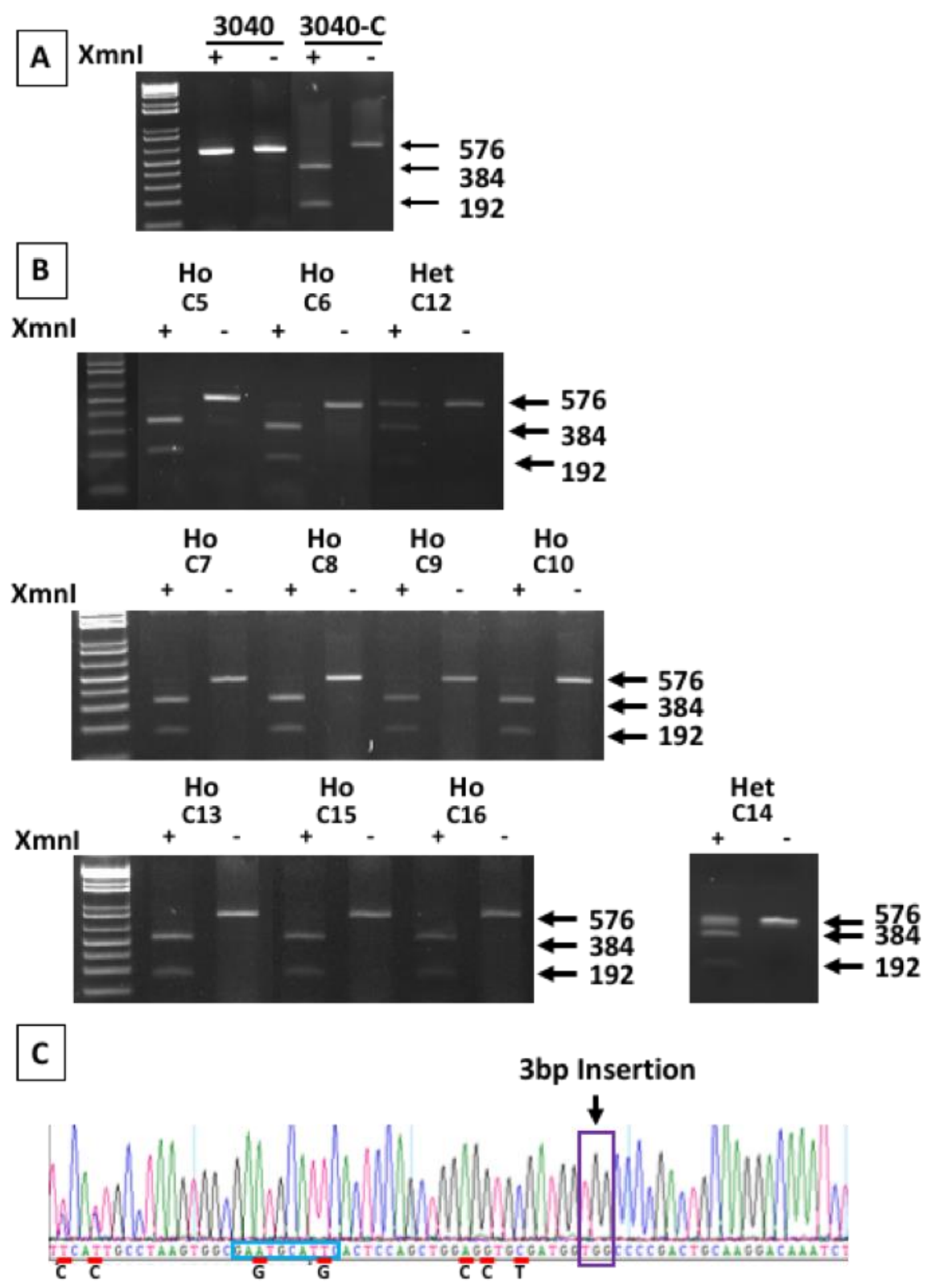

Figure 13. Genetic Analysis 3040-iPSC LDLR Correction by CRISPR/Cas9. (A) RFLP analysis was used to determine 3040-iPSC correction status by restriction digest with XmnI. Non-transfected 3040-iPSC showed no XmnI cleavage (576 bp) in the presence or absence of enzyme. 3040-iPSC clone 1 (3040-C) contained two bands at $384 \mathrm{bp}$ and 192 bp in the presence of XmnI, which remained uncut (576 bp) without enzyme. (B) RFLP analysis confirmed the presence of 9 homozygous (two bands 384 bp and 192 bp) and 2 heterozygous clones (additional band at 576 bp). (C) Sanger sequencing of 3040-C confirmed the permanent insertion of 3-nucleotides in exon 4 of the LDLR in both alleles (purple box). Silent mutations (red line) including the novel XmnI site (blue box) were also integrated into the gene. Bold letters beneath red lines indicate original native nucleotide. Two silent mutations (green box) were introduced in only one allele. Ho= homozygous, Het= heterozygous, $\mathrm{C}=$ clone . 


\section{Genetic Correction Leads to Normalized LDLR Function in 3040-C-HLC.}

Genomic mutations of the LDLR in FH patients can vary from a single nucleotide change to large deletions that will denote the patient into a certain class [234, 235, 254]. The $3 \mathrm{bp}$ deletion in 3040-iPSC are classified as Class IIB FH-Piscataway since the LDLR does not reach maturity and instead is retained in the ER as an immature protein that eventually is degraded by the proteosomal pathway $[92,119,254]$. After treatment with Lovastatin, we observed a robust induction of LDLR expression that was primarily in the form of immature protein in the non-corrected 3040-iPSC, but very little mature protein being detected (Figure 14i, ii $\&$ iii). Excess sterols downregulated LDLR protein production in these cells as expected. In contrast, Lovastatin treatment of the corrected 3040-C-iPSC showed a dramatic shift from predominantly immature protein to predominantly mature protein (Figure 14i, ii \& iii). Again, as expected excess sterol treatment of the corrected cells downregulated LDLR protein expression. When comparing the ratio of mature/immature LDLR with Lovastatin treatment, the corrected cells were greater than 30-fold higher than the non-corrected cells (Figure 14iv) suggesting the CRISPR modification restored the normal LDLR structure which would allow LDLR to proceed to the Golgi for processing and movement to the plasma membrane. Comparing DiI-LDL internalization under the same conditions with the hESC line H1, non-corrected 3040-iPSC internalize very little DiI-LDL even with Lovastatin treatment (perhaps indicating the function of "mature" LDLR in these cells is questionable) (Figure 14A). Confirming the results of the western blot, corrected 3040-C-iPSC internalized DiI-LDL when exposed to Lovastatin and this receptor-mediated endocytosis was abrogated by excess sterols to a similar level as normal H1. These data strongly support the conclusion that there was 
complete genetic correction of the LDLR pathological mutation and normalization of LDL$\mathrm{C}$ receptor-mediated endocytosis. 

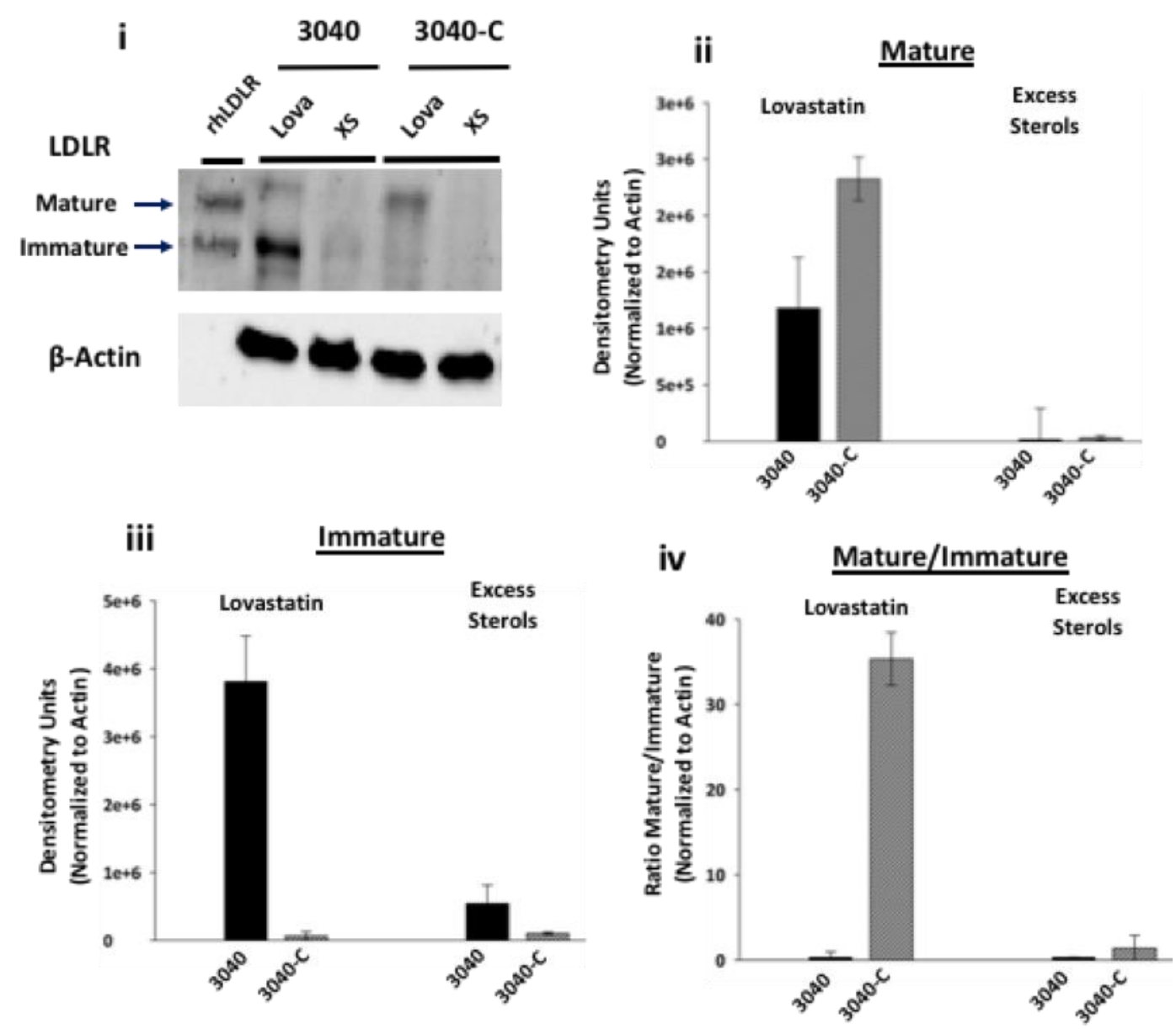

Figure 14. Protein Analysis 3040-iPSC LDLR Correction by CRISPR/Cas9. 3040-C-iPSC and 3040-iPSC were incubated overnight in Lovastatin (Lova) or excess sterols (XS). Western blot (representative image, i) showed 3040-iPSC respond with an upregulation of an immature LDLR after treatment with Lovastatin that is reduced with excess sterols. 3040-C-iPSC had almost no immature LDLR with Lovastatin and increased mature LDLR. Quantification ( $\mathrm{n}=2$ independent experiments) showed a 2-fold increase of mature LDLR in 3040-C treated with Lovastatin compared to 3040 (ii). Immature LDLR was highly expressed in 3040 that was almost lost in 3040-C (iii). Mature/immature LDLR was over 30 times greater in 3040-C than 3040 in Lovastatin that was abrogated in excess sterols (iv). Bars shown as mean \pm S.E.M. 

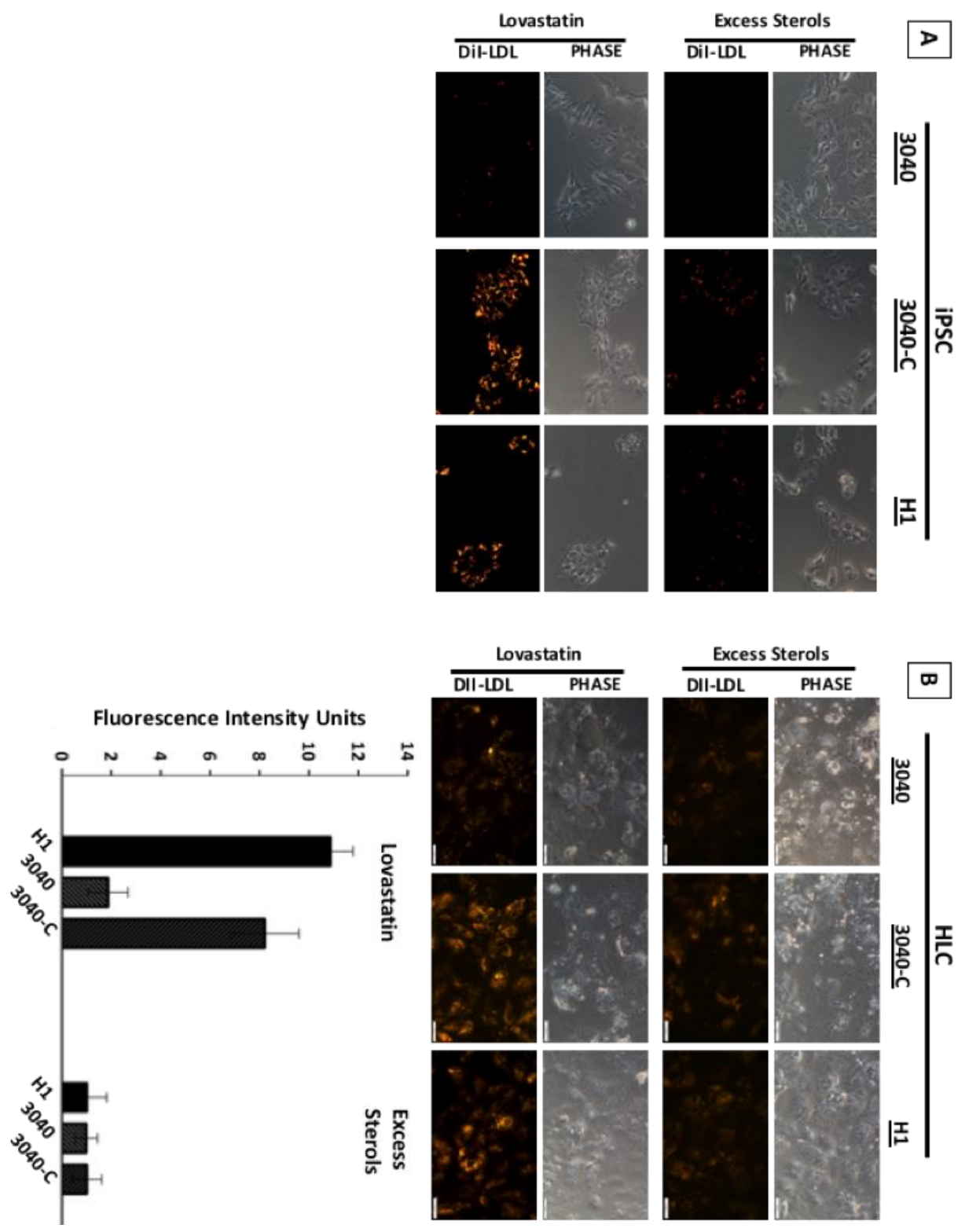

Figure 15. LDLR-Mediated Endocytosis is Restored in Corrected 3040-iPSC and HLC. (A) LDL internalization showed 3040-C-iPSC respond with an increased DiI-LDL internalization similar to control $\mathrm{Hl}$ cells that is almost non-existent in non-corrected 3040-iPSC. LDL uptake is decreased with excess sterols. (B) DiI-LDL uptake was restored in 3040-C-HLC after treatment with Lovastatin that was not obvious in 3040-HLC. H1$H L C$ was used as a control for normal LDLR function. Bar graph ( $n=2$ independent experiments) shows measured fluorescence intensity of DiI-LDL that correlates a 4-fold increase in DiI-LDL uptake in 3040-C-HLC relative to 3040-HLC. Excess sterols were normalized to 1 and Lovastatin normalized to excess sterols. Bars shown as mean \pm S.E.M. $($ Scale bars $=50 \mu \mathrm{m})$. 
The hepatocyte is responsible for production and metabolism of cholesterol which is defective in $\mathrm{FH}$; therefore, we differentiated non-corrected and corrected FH-iPSC to HLC to determine if receptor- mediated endocytosis is corrected and functional in HLC. After differentiation, HLC were treated overnight with either Lovastatin or excess sterols. Fluorescence microscopy of 3040-C-HLC is consistent with 3040-C-iPSC indicating an upregulation in DiI-LDL endocytosis relative to 3040-HLC. H1-HLC control was similar to 3040-C-HLC (Figure 15B). Quantification of DiI-LDL fluorescence intensity showed 3040-C-HLC contained a substantially increased fluorescence intensity compared to 3040HLC (Figure 15B) that was comparable to H1-HLC. For all three cell lines, excess sterols reduced DiI-LDL internalization to a basal level. Taken together, these data demonstrate our CRISPR/Cas9 design successfully corrected the 3bp deletion in the homozygous FH cells, restoring LDLR protein and LDLR-mediated endocytosis.

\section{DISCUSSION}

The novel and important findings of this study are that we have used CRISPR/Cas9 genome editing to permanently correct a homozygous pathological mutation in patientspecific iPSC. We have targeted a Class IIB 3 bp deletion in exon 4 of the LDLR that causes a reduction in receptor activity to less than 5\% of normal. The successful insertion of the missing 3 bp has permanently corrected the genetic mutation and restored the physiological feedback control of receptor expression and cholesterol metabolism. We developed a stepwise enrichment protocol to enhance the probability of isolating corrected clones. The placement of the guides with respect to the mutation site likely made our cells more amenable to correction. Double-positive sorting with GFP and RFP improved 
selection of dual transfected cells while MACS sorting increased the yield of LDLR expressing cells. Our systematic stepwise enrichment protocol allowed for the final isolation of 10 (from 12) homozygous corrected clones (83\%). This procedure is likely adaptable to identifying any corrected surface protein and is a proof-of-concept that CRISPR technology can successfully repair LDLR mutations causing FH.

The estimated prevalence of $\mathrm{FH}$ is 1:250 and 1:250000 for heterozygous and homozygous $\mathrm{FH}$, respectively $[1,237,238]$. It is not uncommon that receptor-defective HoFH patients will present with clinically symptomatic CVD in the first decade of life [18]. A critical issue for these patients is early identification and therapeutic intervention [237]. Because $\mathrm{FH}$ is considered underdiagnosed, screening has been proposed for newborns. For rare HoFH patients, life-style changes and statin/ezetimibe therapy may be insufficient to achieve target LDL-C levels [1]. This requires more aggressive treatment including newly approved pharmacologics (i.e. Mipomersen, Lomitapide), lipid apheresis and even a liver transplant $[15,129,240]$, all of which have their own potential complications. It is also important to recognize that newly approved PCSK9-inhibitors rely on LDLR expression and have a questionable effect on receptor-negative HoFH patients [23]. This supports the need to develop alternative approaches for treating HoFH.

Gene and cell therapies, amenable to monogenic deficiencies [239], to restore cholesterol metabolism homeostasis in FH have been investigated for decades [255]. Gene therapy to deliver an LDLR transgene to the liver has evolved from retrovirus [256] and adenovirus [47] to adeno-associated virus containing liver-specific promoters [241]. The first gene therapy clinical trial using ex-vivo LDLR-transduced autologous hepatocytes showed some benefit to lowering LDL-C, but was halted due to variable metabolic results, 
in particular, in receptor-defective homozygotes [50,51]. A Phase 1/2 clinical trial using AAV-based vector to deliver LDLR is currently approved (NCT02651675). Virus-based gene therapy has demonstrated a capacity to lower cholesterol levels in hypercholesterolemic animal models [257], but issues remain including transgene persistence, vector delivery efficiency, and lack of LDLR expression regulation because of vector size limitations [245]. With regards to cell-based therapy for FH, multiple reports of liver transplantation demonstrate the effectiveness of providing sufficient LDLR-normal hepatocytes to resolve FH hypercholesterolemia [129, 240]. However, liver availability is limited, as are quality hepatocytes. Additionally, transplanted hepatocyte engraftment is very poor unless major insult to the liver is first performed [258]. Together, this demonstrates that issues of transgene and cell delivery for treating HoFH have not been completely solved.

CRISPR/Cas9 genome editing has been demonstrated to be a straight forward methodology for creating mutations and for endogenous mutation repair. It has specifically been used in animal models to repair mutations in the dystrophin gene $D m d$ which causes Duchene muscular dystrophy [192, 259]. Pankowicz and colleagues used CRISPR/Cas9 in a mouse model of hereditary tyrosinaemia type I to delete hydroxyphenylpyruvate dioxygenase $(\mathrm{Hpd})$, which converted the animal to an asymptomatic tyrosinaemia type III [260]. Jarrett, et al [187] delivered CRISPR/Cas9 in an adeno-associated virus to disrupt the LDLR and cause hypercholesterolemia. They co-administered CRISPR/Cas9 to also disrupt the Apob gene, which rescued the hypercholesterolemic phenotype. In a Chinese clinical trial directed by Lu You, CRISPR was used to inactivate the T-cell PDI gene, then delivered back to the patient as a treatment for metastatic non-small cell lung cancer [257]. 
NIH has now approved a Phase I trial for using CRIPSR modified T-cells in cancer patients [261]. Certainly, the immediate benefit of CRISPR genome editing is rapid genetic modification for molecular modeling. Long-term it may also prove of even greater value for therapeutic applications.

CRISPR technology has also been used to modify iPSC $[172,252]$. It can knock-in reporters to specific loci, create knockout models and repair pathological mutations, as we have demonstrated here. The significance of combining CRISPR genome editing with patient specific iPSC is the ability to generate a completely repaired autologous cell source with the potential to differentiate to any cell lineage. It is widely recognized that lack of therapeutic cell sources is one of the major barriers to cell-based therapies and this includes hepatocytes to treat liver diseases [262]. Here, we demonstrate the ability to correct a homozygous deletion in the LDLR and normalize receptor-mediated endocytosis in patient-derived-HoFH-HLC.

The FH-iPSC used in our study is considered a Class IIB FH-Piscataway mutation. This $3 \mathrm{bp}$ in frame deletion results in a missing Gly197 in the highly-conserved binding domain in exon 4 of the LDLR and is highly prevalent in the Ashkenazi Jewish community originating from Lithuania [92]. A class II mutation leads to an improperly folded LDLR since the spacing between cysteine residues becomes abnormal. The immature LDLR is retained in the endoplasmic reticulum and eventually degraded by the proteasome pathway [119]. Once corrected, it is expected that the immature LDLR expression will decrease since the LDLR will properly fold and transition from the endoplasmic reticulum to the Golgi for glycosylation to a fully mature LDLR before it is transferred to the plasma membrane. Our western blot with fibroblast cells (Figure 6B) shows a very low level of 
mature LDLR protein in IMR90 and no immature LDLR in GM03040. This could be due to the fact that these are fibroblast cell lines and do not express LDLR as hepatocytes do. Our western blot data indicates that non-corrected FH-iPSC have a very small mature/immature ratio of LDLR that is normalized in the corrected FH-iPSC. The total LDLR protein (immature + mature) was less in the corrected cells (Figure 14i). The precise reasons for this are unclear, but is likely the result of restoration of feedback regulation, increased receptor recycling, and more efficient LDL-C processing. In noncorrected cells, expression is high and the immature LDLR expression appears to be uncontrolled protein production. Further investigation would be needed to determine if this is unique to a class II mutation.

What we've shown is an efficient way to fully correct a genetic mutation. By using Cas9n the number of off-target mutations is reduced and using specific sgRNA mediates specific on-target cleavage. Intriguingly, only the heterozygous corrected clones introduced indels into their gene. It is known that other off-target modifications may occur in places not predicted in the candidate list (Table 6) due to a variety of reasons including genomic and epigenomic properties that may affect cleavage frequency; therefore, unbiased detection of off-target cleavage such as whole genome sequencing is recommended and would be required before any corrected cell would be delivered to a human patient [263]. Although the CRISPR correction of HoFH-HLC does not solve all cell/gene-based therapy technical hurdles, it provides a platform for autologous cell replacement with a permanent genetic correction to the underlying pathology. CRISPR corrected HoFH-HLC could provide an unlimited source of autologous hepatocytes that 
could be delivered directly to the liver or engineered into a functional liver-like tissue with sufficient cell mass to have a significant effect or even restore LDL-C homeostasis.

To conclude, these data validate the feasibility of using Cas9n, dual sgRNA, and a repair template to correct a genetic mutation in a homozygous FH cell line, which can be differentiated into functional HLC that mediate LDLR endocytosis. In the future, the use of CRISPR-edited iPSC could be tested in patients as is currently being performed in the treatment of cancer [261]. 


\section{CHAPTER III}

\section{MAMMALIAN SPECIES WNT3A DIFFERENTIAL MODULATION OF DERIVED-DEFINITIVE ENDODERM}

\section{$\underline{\text { INTRODUCTION }}$}

Liver dysfunction is caused by a variety of diseases including $\mathrm{FH}$, alpha-1 antitrypsin deficiency, non-alcoholic fatty liver disease, hepatitis B, cancer, and cirrhosis [264]. Although the liver has an innate ability to regenerate itself, the only successful approach to curing any liver disease is through organ transplantation, especially when the damage overtakes regeneration [265]. There are currently over 17,000 patients in the US waiting for a liver transplant [264]. A major problem is the limited amount of donor livers and quality hepatocytes available for patients. Many issues tend to arise post-liver transplantation including donor-host incompatibility, requiring lifelong immunosuppressing medications, causing an increased susceptibility and recurrence of infections [266, 267]. Other problems that may occur include a reversal back to the disease state after transplantation. In patients with $\mathrm{FH}$, long term post-liver transplantation has shown that within 6 months to 2 years, many patients develop cholesterol levels above 200 $\mathrm{mg} / \mathrm{dl}$, requiring high statin therapy [240, 268, 269].

Hepatocytes are the major cell component of the liver [270]. Culturing and expanding primary hepatocytes has proven difficult since their survival depends on the liver microenvironment $[271,272]$. Because of this, cultured hepatocytes begin to 
dedifferentiate and lose their polarity over a short time. An additional key factor that has limited the application of hepatocyte transplantation is the significant cell loss posttransplantation [266, 267, 273-279]. Without immunosuppressive drugs, transplanted allogeneic hepatocytes survive for only 7 to 10 days $[258,267,280]$. Other experimental approaches to address the lack of therapy in diseases like FH have included the delivery of normal LDLR transgenes via viral vectors [51, 244, 245, 281-284]. A clinical trial with HoFH patients utilized ex vivo liver-directed gene therapy and engrafted 3-9 x $10^{9}$ retrovirus-transduced autologous hepatocytes to treat FH [51]. Three of five patients had a decline in LDL-C levels and none developed antibodies to either the LDLR or retroviral proteins. However, $\mathrm{HoFH}$ receptor negative or defective patients did not respond to this treatment. Alternative treatment strategies using induced pluripotent stem cells (iPSC) has become one of the leading research fields for treatment of liver disease and dysfunction [270].

Transcription factors, specifically 4 including Oct4, Sox2, Klf4, and cMyc, are introduced into a differentiated somatic cell to reprogram it into a pluripotent stem cell [242]. The result is an unlimited supply of self-renewable stem-like cells. There are debates as to whether or not iPSC are equivalent to embryonic stem cells (ESC), especially at the RNA and microRNA level $[285,286]$. However, there are many advantages to utilizing iPSC derived from patient cells including their immunological compatibility, limitless supply, and their ability to be differentiated into other cell types like hepatocytes [242, 287, 288].

The Deng lab developed a stepwise protocol for differentiation of iPSC into hepatocyte-like cells (HLC) [250]. This protocol used a 5-stage protocol that involved 
endoderm induction, hepatic specification, hepatoblast expansion, hepatic maturation, and matured hepatocytes. Their process required the sequential use of growth factors for the gradual progression from endoderm to mature hepatocyte. The critical factors were Activin A (AA), bone morphogenetic protein 2 (BMP2), fibroblast growth factor 4 (FGF4), hepatocyte growth factor (HGF), and Oncostatin M (OSM). These 5 factors were included among 8 (keratinocyte growth factor, Dexamethasone, N2) different components necessary in the 5-stage, 21-day protocol for differentiation of iPSC to HLC [250]. By the end of 3 weeks of differentiation, the HLC expressed hepatic markers including immature hepatocyte marker alpha-fetoprotein (AFP), mature hepatocyte marker albumin, and hepatocyte plasma membrane marker cytokeratin 18. In addition, the cells exhibited activity of hepatocytes including glycogen and urea synthesis and albumin secretion [250].

Duncan and colleagues modified this protocol $[289,290]$ so that only 4 stages were needed but more importantly, the growth factors required were decreased to 5 (AA, BMP2, FGF4, HGF, and OSM). In addition, the environmental conditions were modified to a hypoxic state ( $4 \%$ oxygen, $5 \%$ carbon dioxide). At the end of this differentiation, $70 \%$ to $90 \%$ of cells expressed albumin with a decrease in AFP expression indicating these humanderived HLCs when injected into mouse livers integrated into the existing mouse parenchyma [289]. Both of these differentiation protocols were successful in differentiating human iPSC to HLC [289, 290].

As mentioned in the previous chapter, ESC/iPSC can differentiate into 3 germ layers: endoderm, mesoderm, and ectoderm. The proper development of the primitive ventral endoderm that forms the liver requires Wnt3a [291]. Wnt3a is a member of highly conserved glycoprotein family members that are necessary in normal development [292]. 
Knockout of Wnt3a resulted in mouse ESC that failed to express mesoderm and endoderm genes suggesting the inability to generate a primitive streak [293].

Bearing this in mind, the lab of Dr. David Hay designed an HLC-differentiation protocol that mimicked the developing embryo resulting in a homogenous differentiation HLC population [294]. Eventually they developed a 3-stage protocol that used AA and Wnt3a in stage 1, no growth factors in stage 2, and HGF and OSM in stage 3 [295]. This yielded 90\% albumin-expressing HLC.

Our lab has previously differentiated iPSC to HLC [189, 245] using the 5-stage Song protocol [250]. This resulted in efficient generation of HLC as shown in Figure 9. However, the cell yield was low. Differentiation to definitive endoderm is considered the critical stage to HLC production. It is here that will decide the fate of whether or not cells differentiate to hepatocytes and if they do so in an efficient manner. Therefore, we investigated which protocol was the most efficient at producing endoderm.

\section{MATERIALS AND METHODS}

Cell Culture. H1 (WiCell, Madison, WI) were cultured on hESC-Qualified Matrigel coated plates (BD Biosciences) in mTeSR1 with media changed daily (STEMCELL Technologies) [245]. Cells were passaged using Gentle Cell Dissociation Buffer (GCDB; STEMCELL Technologies) with 10mM Rock inhibitor (Selleck Chemical) and maintained at $37^{\circ} \mathrm{C}$ and $5 \% \mathrm{CO}_{2}$ [245].

Hepatocyte Differentiation. H1-ESC underwent directed differentiation to definitive endoderm as outlined in Figure 16 below. 

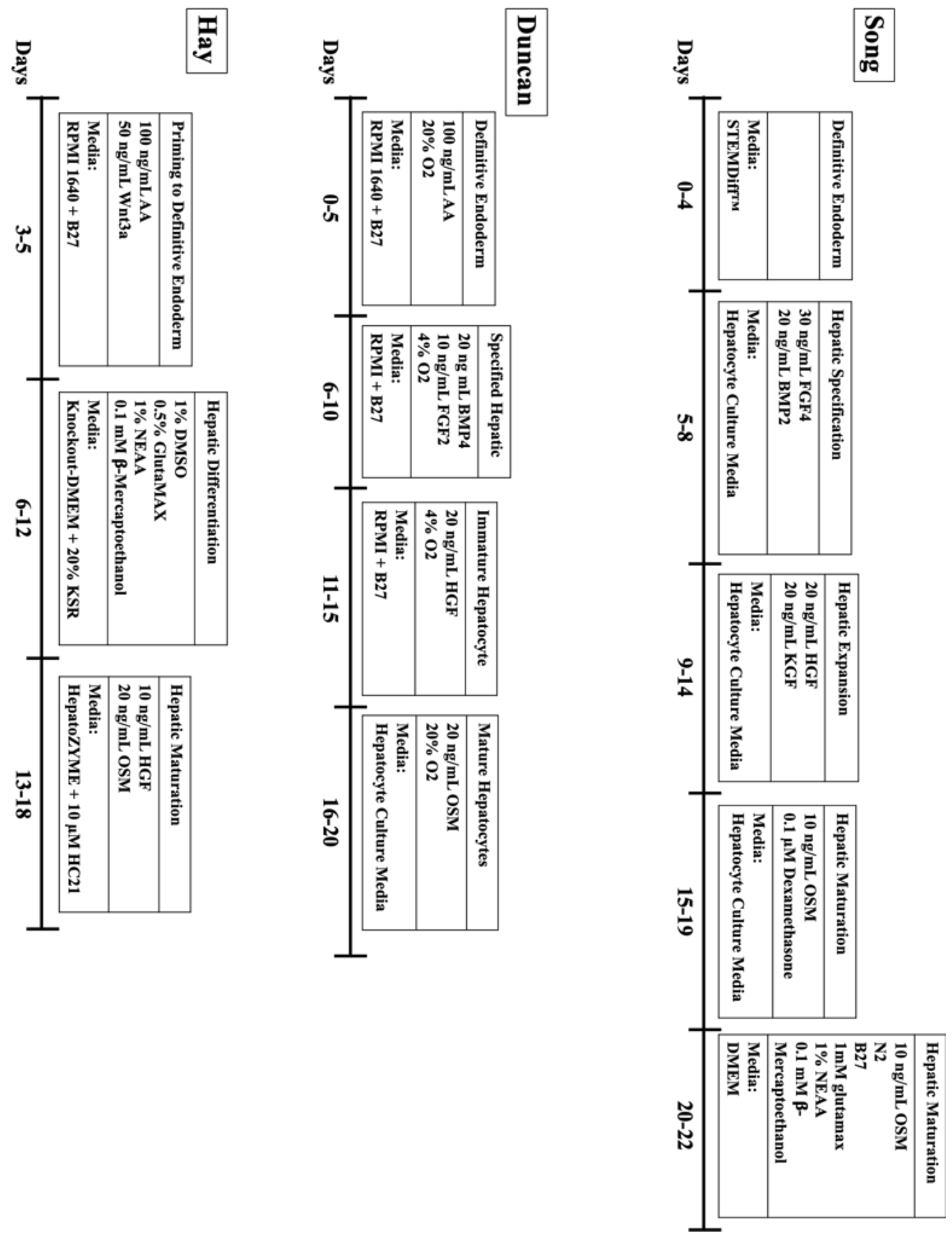

Figure 16. Hepatocyte-Like Cell (HLC) Differentiation Protocol. H1 stem cells underwent differentiation to the end of stage 1 or definitive endoderm. Song stage 1 has been modified for use of commercially available StemDiff ${ }^{\mathrm{TM}}$ (STEMCELL Technologies, Vancouver, Canada) for 4 days. Duncan requires the use of AA, BMP4, FGF2, HGF, and OSM. Hay utilizes AA and Wnt3a in the definitive endoderm stage and only adds OSM and HGF in the final stage. 
Fluorescence Activated Cell Sorting (FACS). After differentiation to definitive endoderm, cells were dissociated to single-cells, counted, centrifuged, and re-suspended at $<10^{6}$ cells in $100 \mu \mathrm{L}$ stain buffer and kept on ice. The same was done with control HepG2 and non-differentiated $\mathrm{H} 1$ cells. The cells were incubated in their appropriate isotype controls or antibodies (Table 7) for 30 minutes at $4^{\circ} \mathrm{C}$ protected from light. After 30 minutes, cells were washed twice with stain buffer. Cells were re-suspended in $300 \mu \mathrm{L}$ stain buffer. The cells were run through the BD LSRII Cytometer (BD Biosciences) and analyzed via FlowJo (BD Biosciences).

mWnt3a and hWnt3a Dose Course. H1-ESC were differentiated to definitive endoderm following Hay's stage 1 protocol (Figure 11). Mouse Wnt3a (mWnt3a; Peprotech) or human Wnt3a (hWnt3a; R\&D) were added to definitive endoderm media at various concentrations $[0,10,25,50 \mathrm{ng} / \mathrm{mL}]$. After 5 days, cells were collected and analyzed via qPCR for definitive endoderm markers.

mWnt3a and hWnt3a Time Course. H1-ESC underwent directed differentiation to definitive endoderm following Hay's stage 1 definitive endoderm (Figure 11). $50 \mathrm{ng} / \mathrm{mL}$ of mWnt3a or hWnt3a were added to definitive endoderm media. Cells were collected at time point $0,1,3$, and 5 days and analyzed via qPCR for definitive endoderm markers.

Quantitative PCR analysis. At the end of stage 1, H1 cells were lysed using 100 $\mu \mathrm{L}$ of $0.1 \% \beta$-mercaptoethanol in RLT Buffer (Qiagen). The lysates were purified with Qiashredder and RNeasy kits (Qiagen) according to the manufacturer's instructions. RNA was quantified with a NanoDrop One Spectrophotometer (ThermoFisher). cDNA was synthesized using $1 \mu \mathrm{g}$ RNA with SuperScript IV reverse transcriptase (Invitrogen) in a 20 $\mu \mathrm{L}$ volume. The qPCR was performed using Fast SYBR Green Master Mix 
(ThermoFisher) with primers obtained from Integrated DNA Technologies (IDT) (Table 8). Reactions were run on the StepOnePlus Real-Time PCR System (ThermoFisher). Raw data was analyzed in Microsoft Excel and graphs produced in GraphPad's Prism 8.

\section{Table 7}

List of Antibodies for FACS

\begin{tabular}{|c|c|c|c|}
\hline Tube & Particle/Cell & APC & AF700 \\
\hline Bead (+) & (+) bead/ (+) IgG bead & -- & -- \\
\hline Bead Tra-1-81 & $\begin{array}{l}\text { (+) bead/ (+) IgG bead + } \\
\text { Tra-1-81-APC in 1ml SB }\end{array}$ & $\begin{array}{l}10 \mu \mathrm{l} \mathrm{Tra-1-81} \\
\text { APC }(\mathrm{R} \& D)\end{array}$ & -- \\
\hline Bead CXCR4 & $\begin{array}{l}(+) \text { bead/ (+) IgG bead + } \\
\text { CXCR4-AF700 in 1ml SB }\end{array}$ & -- & $\begin{array}{l}5 \mu \mathrm{l} \mathrm{CXCR4} \mathrm{AF700} \\
(\mathrm{R \& D})\end{array}$ \\
\hline Control & H1 & Tra 1-81 APC & CXCR4 AF700 \\
\hline Control & H1 & $\begin{array}{l}\text { Mouse IgM APC } \\
\text { (R\&D) }\end{array}$ & $\begin{array}{l}\text { Mouse IgG2b } \\
\text { AF700 (R\&D) }\end{array}$ \\
\hline \multirow[t]{2}{*}{ Samples } & $\begin{array}{l}\text { Hay DE, Duncan DE, } \\
\text { Song DE }\end{array}$ & Tra 1-81 APC & CXCR4 AF700 \\
\hline & $\begin{array}{l}\text { Hay DE, Duncan DE, } \\
\text { Song DE }\end{array}$ & IgM APC & IgG AF700 \\
\hline
\end{tabular}

\section{Table 8}

List of Primers Used for qPCR Analysis of Definitive Endoderm

\begin{tabular}{|l|l|l|l|}
\hline Gene & Primer 1 $\left(5^{\prime} \rightarrow \mathbf{3}^{\prime}\right)$ & Primer $2\left(5^{\prime} \rightarrow \mathbf{3}^{\prime}\right)$ & $\begin{array}{l}\text { Product } \\
\text { Size } \\
(\mathrm{bp})\end{array}$ \\
\hline Oct4 & TGATCCTCGGACCTGGCTAA & AACCACACTCGGACCACATC & $\mathbf{1 8 0}$ \\
\hline Sox17 & CCGCGGTATATTACTGCAACTA & CACCCAGGACAACATTTCTTTG & $\mathbf{1 5 2}$ \\
\hline Hnf4a & GGCCAAGTACATCCCAGCTT & TCATTGCCTAGGAGCAGCAC & $\mathbf{1 7 0}$ \\
\hline P0 & TCGACAATGGCAGCATCTAC & ATCCGTCTCCACAGACAAGG & $\mathbf{2 0 0}$ \\
\hline
\end{tabular}




\section{RESULTS}

Activin A and Wnt3a increase CXCR4+ Definitive Endoderm Cells. We began by investigating which of the 3 endoderm differentiation protocols would deliver the highest rate of this cell type. Efficient production of endoderm requires expression of the endoderm markers Sox17 and CXCR4 [296]. Markers for ESC/iPSC like Tra-1-81 can distinguish cells that have yet to begin differentiation [297]. We plated H1-ESC onto 35 mm dishes and began each of the differentiation processes according to Figure 16. To note, we did not have a chamber available to maintain cells at $4 \%$ oxygen prior to or throughout the course of differentiation as suggested by Duncan [289]. Instead, we focused on treating H1 with only Activin A for the 5-day differentiation. We observed significant cell death at day 2 with the Song/StemDiff and at day 3 with Duncan and Hay. However, cells grew in most of the plates at the end of stage 1 . We collected the cells for analysis of Tra-1-81 and CXCR4 expression by FACS.

Our H1 non-differentiated control expressed only Tra-1-81 (Figure 17A) and no cell-surface CXCR4. In contrast, all 3 endoderm differentiation procedures resulted in expression of CXCR4 (Figure 17B, C, D, bottom right panel). However, there also appeared to a substantial number of Tra-1-81 expressing cells present in all 3 cell cultures (Figure 17B, C, D, bottom center panel). Compiling the data for Tra-1-81 and CXCR4 into single graphs indicated that indeed for all the differentiation protocols there were many Tra-1-81 expressing cells, indicating they are still stem cells (Figure 18). In comparing each protocol, Song/StemDiff appeared to have lowest expression of Tra-1-81 and an increase in CXCR4 (Figure 18, blue). The Hay protocol resulted in a shift to the right for both Tra-1-81 and CXCR4 compared to Song/StemDiff (Figure 18, green). Cells in 
Duncan's modified protocol had the largest shift to the right in both Tra-1-81 and CXCR4 expressing cells indicating that while a large population were differentiating to endoderm, a large population were still stem cells too (Figure 18, yellow). Although more cells expressed CXCR4, the Tra-1-81 expression was greater than that of $\mathrm{H} 1$ control nondifferentiated cells. Since Hay's protocol resulted in the greatest number of CXCR4 expressing cells, we used this protocol for the rest of this study.

Wnt3a species and dose impact differentiation of cells to definitive endoderm.

The Hay protocol calls for the use of $50 \mathrm{ng} / \mathrm{mL}$ of recombinant mouse Wnt $3 \mathrm{a}$ (mWnt3a) purchased from Peprotech [295]. Other sources have used human Wnt3a at lower concentrations [298] although it has been shown that recombinant mouse Wnt3a (mWnt3a) exhibited higher Wnt signaling activity than hWnt3a [299]. We began by investigating whether mWnt $3 \mathrm{a}$ or $\mathrm{hWnt} 3 \mathrm{a}$ would be most efficient for endoderm. $\mathrm{H} 1$ cells were plated and differentiated using Hay's protocol (Figure 16) with the modification of either hWnt3a or $\mathrm{mWnt} 3 \mathrm{a}$ at concentrations of $0,10,25$, and $50 \mathrm{ng} / \mathrm{mL}$. After 5 days, the cells were collected and qPCR was done to assess mRNA transcript levels for Oct4, Sox17, and Hnf4a, which are common markers for distinguishing stem cells from definitive endoderm (Figure 19). 

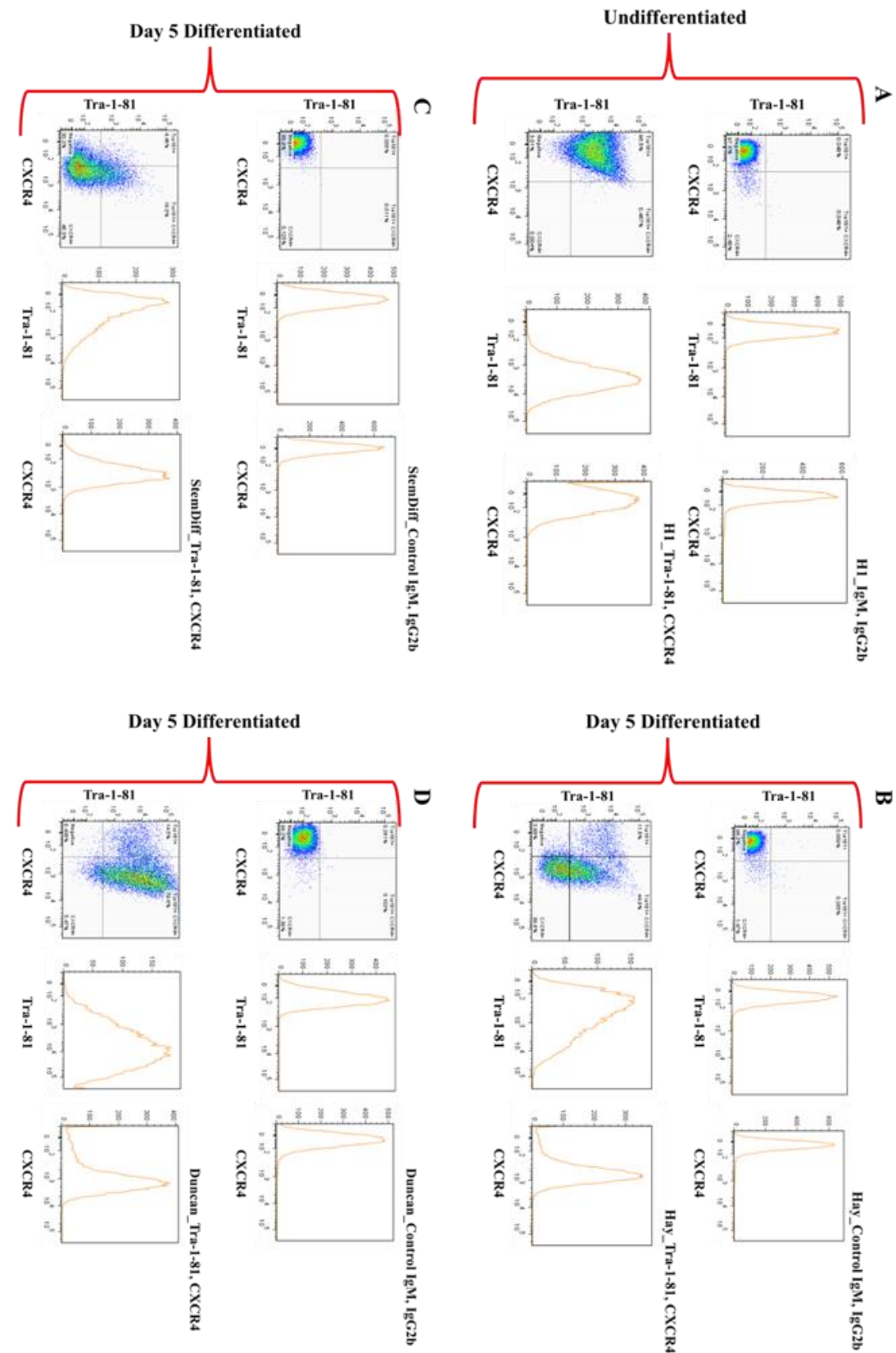

Figure 17. Differentiation for Endoderm. H1 stem cells were differentiated using 3 different protocols and analyzed for expression of stem cell or definitive endoderm markers by FACS. In the top panels, control IgM and IgG2b are not expressed in the cell (A) Control H1 non-differentiated stem cells bottom panels: H1 express no CXCR4 and are all Tra-181 positive. (B) Hay protocol for definitive endoderm: bottom panels: some cells still express Tra-1-81 and there is clear expression of CXCR4 positive cells; most cells are alive. (C) Song/StemDiff protocol for definitive endoderm: bottom panels: expression of CXCR4 positive cells is greater than Tra-1-81 but there is also increased cell death. (D) Duncan protocol for definitive endoderm: bottom panels: CXCR4 positive cells present but a larger number of Tra-1-81 cells still present in culture. $(n=1)$ 
Expression of octamer-binding transcription factor 4 (OCT4) is an indicative pluripotence marker while sex determining region $\mathrm{Y}$ box 17 (SOX17) is a definitive endoderm marker [250]. Hepatic lineage specification is indicated by expression of hepatocyte nuclear factor 4a (HNF4A) [250]. Oct4 expression was highest in nondifferentiated cells in both groups. Noticeably, hWnt3a treated cells had low expression of Oct4 across all doses that was only present with Activin-A in the mWnt3a treated culture (Figure 19A). Expression of Sox 17 was upregulated in mWnt3a treated culture and appeared to decrease with increased dose (Figure 19B). hWnt3a treated culture had reduced Sox17 expression, while its Hnf4a transcript levels were elevated compared to mWnt3a treated culture in all doses (Figure 19C). Of interest, Oct4 expression levels were 10 times higher than Sox17 and Hnf4a levels. Graphs depicting this indicate just how low expression of definitive endoderm and hepatic markers can be in a mixed cell culture (Figure 19D, F).

Because definitive endoderm differentiation occurs over 5 days, we examined a time course for changes in expression of iPSC markers recession and endoderm marker appearance. Similar to the dose curve data (Figure 19), Oct4 expression was higher in $\mathrm{mWnt} 3 \mathrm{a}$ treated culture than $\mathrm{hWn}$ 3a treated culture. Still, Oct 4 decreased greatly by day 3 in both cultures (Figure 20A). Sox 17 expression was 10-fold higher in mWnt3a treated culture than hWnt3a treated cells, particularly by day 3 across all doses in mWnt3a treated cells. Hnf4a transcripts were also upregulated in mWnt3a treated culture as the dose days progressed. This was surprising in that hWnt3a treated culture had an increased Hnf4a at day 5 across all doses in Figure 14C. This was completed with a sample of 1 and requires repeating. 


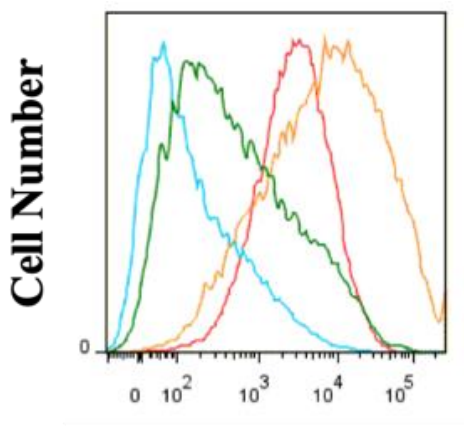

Tra-1-81

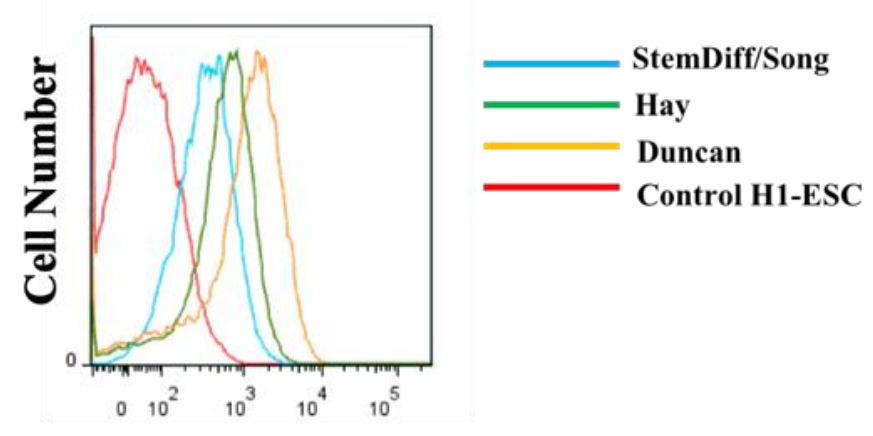

CXCR4

Figure 18. All Protocols Result in a Mixed Population of Tra-1-81 and CXCR4. Control (red) non-differentiated cells expressed only Tra-1-81. StemDiff/Song (blue) showed lower Tra-1-81 and higher CXCR4 expression but an overall smaller population of total cells. Duncan (yellow) had the highest number of cells expressing both Tra-1-81 and CXCR4. Hay (green) had a lower number of Tra-1-81 positive cells than Duncan and similar levels of CXCR4. $(n=1)$ 

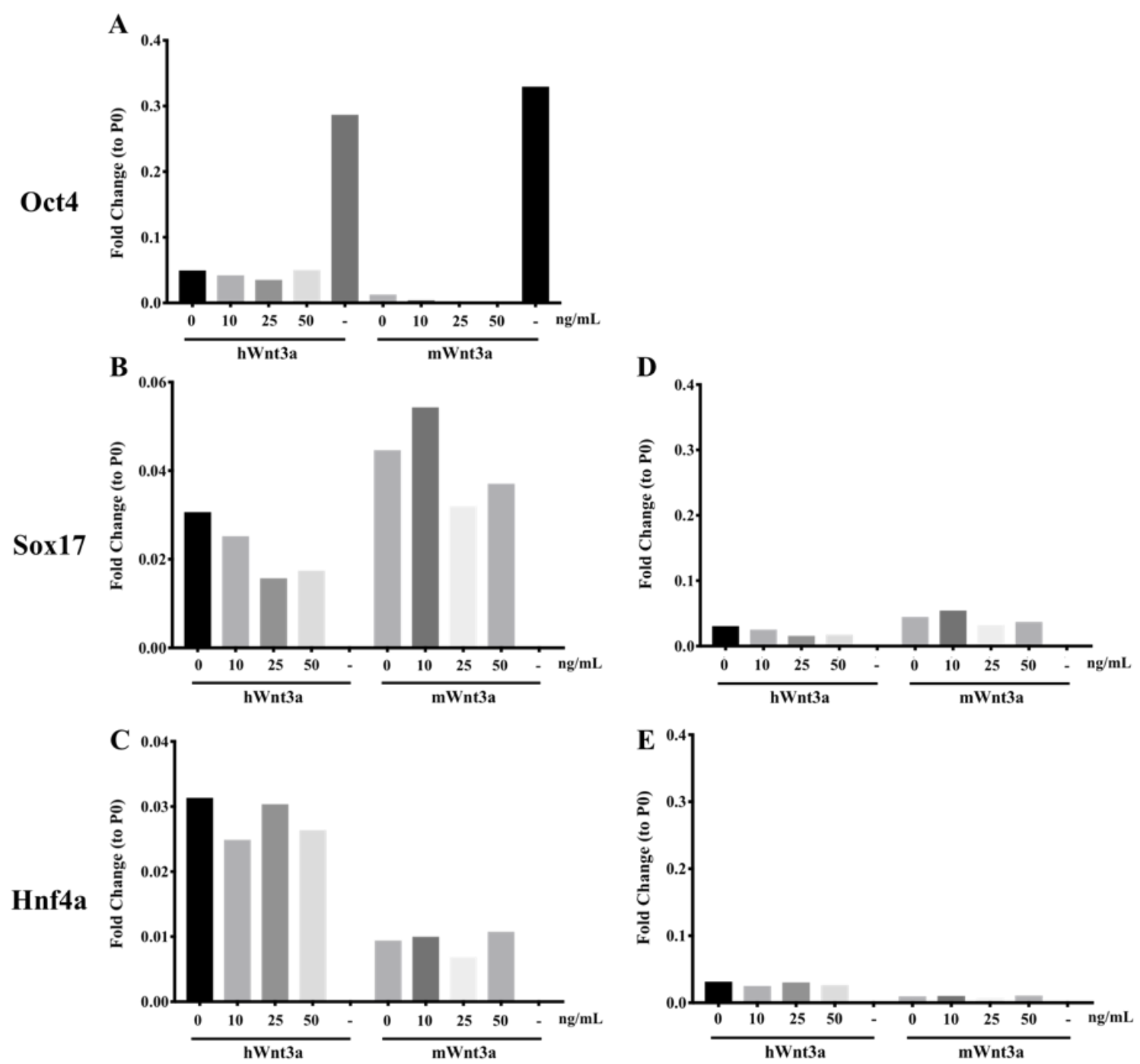

Figure 19. Human Wnt3a Causes Increased Hnf4a Expression Compared to mWnt3a. Stage-specific markers were tested at the mRNA expression level (POU4F1, SOX17, HNF4A). (A) Oct4 expression was highest in non-differentiated cells. (B) Sox 17 definitive endoderm was increased in $10 \mathrm{ng} / \mathrm{mL} \mathrm{mWnt} 3 \mathrm{a}$ cells. (C) Hnf4a had high expression in $\mathrm{hWnt3a}$ culture across all doses. (D,E) Sox 17, Hnf4a expression modified to fit $\mathrm{Y}$-axis of Oct 4 to distinguish the low expression of these 2 markers. 
mWnt3a

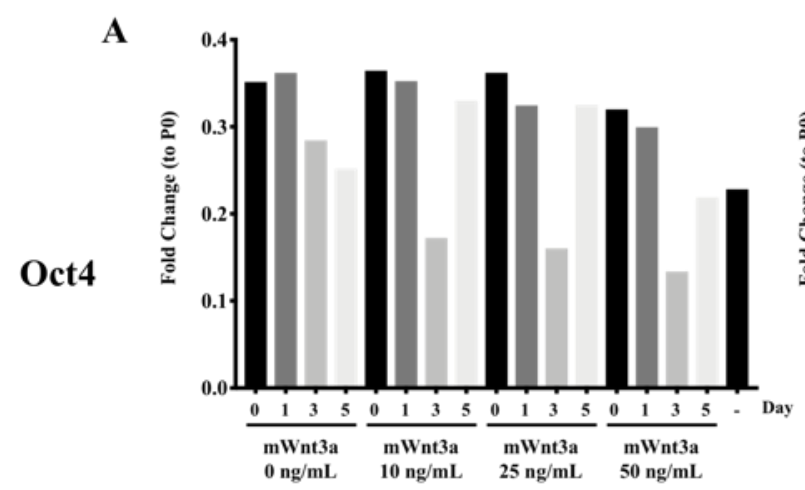

B

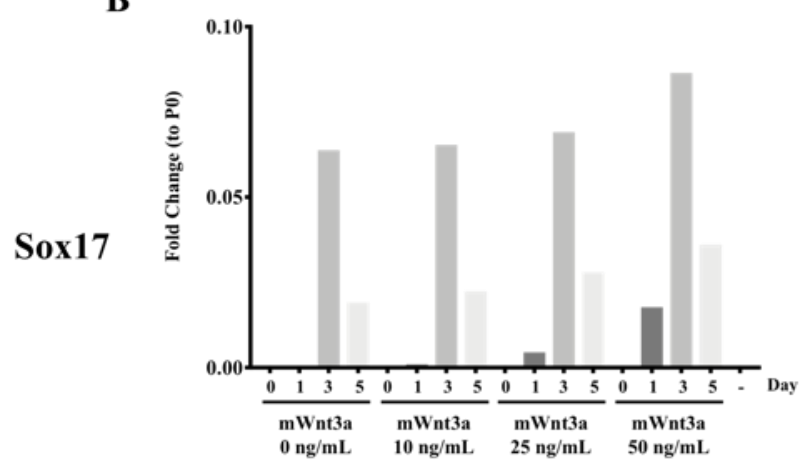

C

Hnf4a

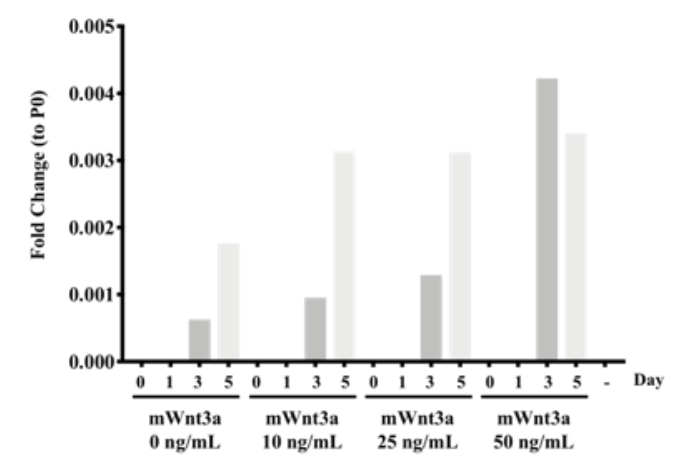

hWnt3a
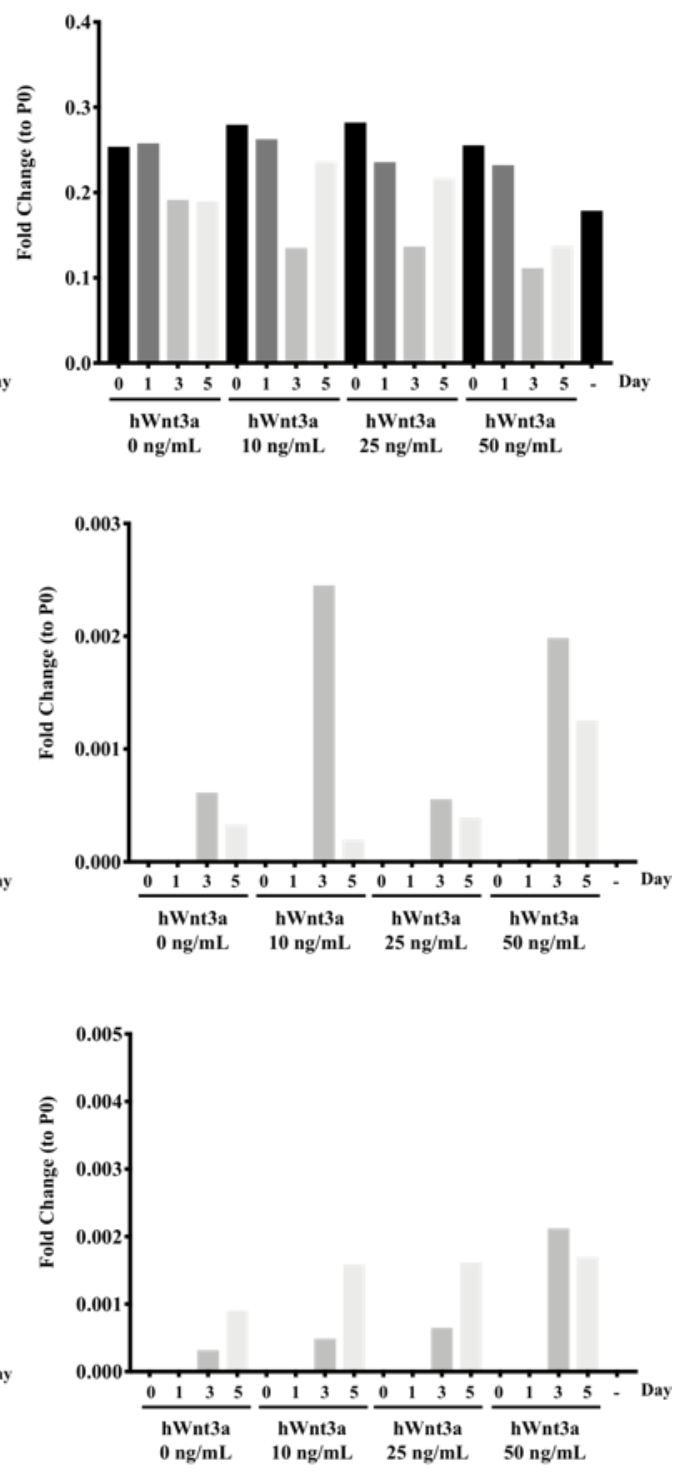

Figure 20. Hnf4a Expression Occurs by Day 5 in Endoderm Differentiation. (A) After time course of 5 days, Oct 4 expression was highest in $\mathrm{mWnt} 3 \mathrm{a}$ at all doses but began to decrease by day 3 in culture. The same pattern was observed in hWnt3a culture. (B) Sox17 was elevated at day 3 in both mWnt3a and hWnt3a at all doses and begins to decrease at day 5 . (C) Hnf4a had high expression in $\mathrm{m} \mathrm{Wnt3a}$ at day 5 as did hWnt3a.

\section{$\underline{\text { DISCUSSION }}$}

There are many factors to consider when beginning the differentiation process of cells. The starting confluence, the plating of single cells over clumps/colonies of cells, and 
even the logarithmic phase of the ESC/iPSC growth prior to passaging for differentiation must all be scrutinized to reliably generate healthy HLC $[289,300]$. A protocol described in one paper effectively producing functional HLC does not mean that this will occur universally in all labs. These protocols are like recipes that must be examined and optimized for in your own kitchen. With this in mind, we sought optimize conditions for differentiation of our ESC to HLCs.

The main finding of this report is that $25 \mathrm{ng} / \mathrm{mL}$ and $50 \mathrm{ng} / \mathrm{mL}$ of $\mathrm{hWnt} 3 \mathrm{a}$ and mWnt3a with the Hay protocol both upregulate expression of endoderm and hepatic markers (Sox17 and Hnf4a) reaching similar levels [295]. This indicates that both may be sufficient in producing definitive endoderm. To confirm this result, the experiment needs additional replication. In addition, differentiation to HLC would provide evidence, if favoring one or the other was optimal.

We observed that Duncan's protocol yielded higher numbers of H1-CXCR4 expressing cells; however, the number of ESC Tra-1-81 positive cells was just as high (Figure 18D). We did not follow the protocol precisely since we did not have access to a cell chamber to maintain hypoxic oxygen levels. This may be the key to reducing the number of non-differentiated cells. It has been demonstrated that hypoxic oxygen levels at $1 \%$ significantly increase Activin A-induced definitive endoderm cells compared to $21 \%$ normoxia in mouse ESC [301]. With a controlled chamber for oxygen, we could determine if Wnt3a is necessary for differentiation of our iPSC to HLC.

Despite this work, it is known that HLCs derived from iPSC will still present an immature hepatocyte phenotype, which would limit their use in clinical therapy. The extracellular matrix $(\mathrm{ECM})$ is regarded as the foundation that can make or break derived 
hepatocytes potential of becoming fully functioning mature hepatocytes. Matrigel is the main ECM used for both stem cell culture and differentiation [290, 299, 302]. Matrigel, unfortunately, varies from batch to batch and is still undefined with respect to various components [303]. It also contains animal derivatives that would make it difficult to use in clinical work. An alternative to Matrigel could be the use of laminins. Laminins (LN) are glycoproteins that are part of the ECM. They are found in the ECM of the embryo. Hay's lab utilized two laminins, LN521 and LN111, to derive HLC from iPSC [304]. These two laminins are present in the liver stem cell niche and have been shown to drive endoderm and liver stem cell differentiation [305, 306]. LN521 and LN111/LN521 mixture increased the number of cells with morphological appearance of HLC compared to the use of Matrigel. Additionally, staining for bile canaliculi organization demonstrated a functional bile canaliculi on the LN521 and LN111/LN521 mix that was present on Matrigel [304].

The use of patient-specific iPSC for studies in disease modeling, drug toxicity, and organoid transplantation would require a highly pure culture of definitive endoderm in the initial stage of differentiation. Taking into consideration these factors involved can help us better understand and create protocols that can be useful in our research. 


\section{CHAPTER IV}

\section{STATIN-INDUCED ACCUMULATION OF CLASS II IMMATURE LDL-RECEPTOR \\ IN FAMILIAL HYPERCHOLESTEROLEMIA DOES NOT ACTIVATE THE UNFOLDED PROTEIN RESPONSE}

\section{$\underline{\text { INTRODUCTION }}$}

Familial hypercholesterolemia $(\mathrm{FH})$ is an autosomal dominant disease primarily caused by mutations in the LDL receptor (LDLR) gene leading to premature cardiovascular disease (CVD) [17, 234-236]. LDLR activity ranges with disease severity and is based on the mutation within the LDLR [92]. There are over 1200 LDLR mutations identified that are categorized into six classes [236, 239]. As of 2012, greater than $50 \%$ have been described as class II or transport defective mutations [92, 98]. After translation, a newly synthesized and unfolded LDLR is processed in the ER as a partially glycosylated precursor of 120-kDa [98], aided by ER chaperones glucose-regulated protein 78 (Grp78), receptor associated protein (RAP) [98, 106, 307] and Mesoderm Development (MESD) [110]. The LDLR is then transported to the Golgi where N-and O-linked sugars are added, increasing the molecular weight to $160-\mathrm{kDa}[116,117]$. Class II mutations are commonly referred to as a "folding or conformational disease" $[98,120,121]$ because these mutations result in a misfolded LDLR that is either unable or has a less than 5\% rate of leaving the ER for the Golgi [92]. 
The molecular mechanisms responsible for LDLR folding and maturation is still unclear. The quality control system of the ER ensures that newly synthesized proteins only leave the compartment when their folding criteria have been met [208, 209, 211]. Chaperones expressed in the ER play a significant role in the protein folding process [105, 214]. General chaperone Grp78 transiently binds the LDLR and aids in its proper folding under normal conditions $[124,125,215]$. This correlates with studies of Grp78 acting selectively in retaining proteins in the ER [215]. This quality control ensures that only properly folded proteins exit the ER for the Golgi, while misfolded proteins are retained in the ER for further processing. If unable to be corrected, misfolded proteins in the ER can accumulate and cause ER stress, activating the unfolded protein response (UPR) [213, 217, 218]. The UPR's major role is to maintain protein homeostasis in the presence of accumulated un/misfolded proteins. The 3 major stress sensor pathways in UPR activation are inositol-requiring transmembrane kinase/endonuclease (IRE1 $\alpha$ ), PKR-like ER kinase (PERK), and activating transcription factor 6 (ATF6) [213]. The UPR works to alleviate ER stress by upregulating the folding capacity through controlling expression of transcription factors and other downstream targets that specifically mediate protein folding, ER-Golgi trafficking, organelle biogenesis, and ER-associated degradation (ERAD) [213]. Also classified as a misfolded protein disease [98], there is very limited research into class II LDLR misfolding, primarily because of the accelerated devastating effect FH has on the cardiovascular system. Evidence presented in the literature suggests that ER stress occurs in FH class II mutations because of accumulating misfolded LDLR [124, 125]. We reprogrammed class II FH fibroblasts with a homozygous 3 bp deletion in LDLR exon 4 (GM03040) with <5\% receptor activity to iPSC [189]. We utilized the genome 
editing tool, clustered-regularly-interspaced-short-palindromic-repeats/CRISPRassociated 9 (CRISPR/Cas9), to permanently correct the 3 bp deletion in the LDLR. The successful correction restored physiological feedback control of receptor expression [189]. C-iPSC confirmed restoration of the mature LDLR protein and normalization of receptormediated LDL internalization compared to NC-iPSC. Prior to correction, we observed a significant accumulation of immature LDLR protein upon treatment with statin in NCiPSC [189]. Understanding that ER stress activates UPR due to accumulation of misfolded proteins and that overexpressed class II LDLR mutants express ER stress markers, we questioned how statin therapy affects FH class II LDLR function. Using our FH class II LDLR corrected and non-corrected model as a tool, we hypothesized that class II FH cells differentiated to hepatocyte-like cells (HLC) activate UPR when treated with statins and this effect is diminished with correction of the LDLR.

\section{MATERIALS AND METHODS}

Cell Culture and Hepatocyte Differentiation. Reprogrammed human induced pluripotent stem cells (iPSC), 3040-iPSC and 3040-C-iPSC (derived from Coriell Cell Repository GM03040 fibroblasts) [Add Omer et al here], were cultured on hESC-Qualified Matrigel coated plates (BD Biosciences, San Jose, CA) in mTeSR1 with media changed daily (STEMCELL Technologies, Vancouver, Canada) [245]. Cells were passaged using Versene (ThermoFisher, Waltham, MA) with $10 \mu \mathrm{M}$ Rock inhibitor (Selleck Chemical, Houston, TX) and maintained at $37^{\circ} \mathrm{C}$ and $5 \% \mathrm{CO}_{2}$ [245]. $\mathrm{H} 1$ cells (WA01/NIH 0043, WiCell, Madison, WI) were cultured as the iPSC.

All three cell lines underwent directed differentiation to hepatocyte-like cells as reported previously [308]. Briefly, iPSC were plated on hESC-Qualified Matrigel coated 
$60 \mathrm{~mm}$ plates at $5 \times 10^{5}$ cells. The next day, stage 1 definitive endoderm differentiation was initiated by replacing stem cell media with stage 1 differentiation media (RPMI1640 (Invitrogen, Waltham, MA) with B27 (1X, Invitrogen)) supplemented with human Activin A (100 ng/mL, Peprotech, Rocky Hill, NJ) and human Wnt3a (50 ng/mL; R\&D Systems, Minneapolis, MN). Media was changed daily for 5 days. This was followed with a 5-day culture in stage 2 hepatoblast media (Knock Out Dulbecco's Modified Eagle's medium (KO-DMEM, Invitrogen), 20\% knockout serum replacement (KSR, Invitrogen), 0.5X GlutaMAX (Invitrogen), $1 \%$ nonessential amino acids (Invitrogen), $0.1 \mathrm{mM} \quad \beta$ mercaptoethanol (ThermoFisher), and 1\% DMSO (v/v) (ThermoFisher) with media replacement every other day. Cells were finally cultured in stage 3 hepatocyte maturation media for 11 days (HepatoZYME (Invitrogen) with $10 \mu \mathrm{M}$ hydrocortisone 21hemisuccinate (Sigma-Aldrich), 0.5X GlutaMAX supplemented with human hepatocyte growth factor (HGF, $10 \mathrm{ng} / \mathrm{mL}$, Peprotech) and human Oncostatin M (OSM, $20 \mathrm{ng} / \mathrm{mL}$, Peprotech)). The media was changed every other day.

Quantitative PCR analysis. The iPSC or HLC were starved overnight in 5\% lipoprotein-deficient serum (LPDS, ThermoFisher) media supplemented with $5 \mu \mathrm{M}$ Rosuvastatin (EMD Millipore, Burlington, MA) or excess sterols $(10 \mu \mathrm{g} / \mathrm{mL}$ cholesterol and $5 \mu \mathrm{g} / \mathrm{mL}$ 25-hydroxycholesterol; Sigma-Aldrich). Positive control cells were also treated with $5 \mu \mathrm{g} / \mathrm{mL}$ Tunicamycin (Invitrogen) for 4 hours. Control cells were treated with DMSO overnight. At the end of treatment, iPSC and HLC were lysed using $150 \mu \mathrm{L}$ of $0.1 \% \beta$-mercaptoethanol in RLT Buffer (Qiagen, Valencia, CA). The lysates were purified with Qiashredder and RNeasy kits (Qiagen) according to the manufacturer's instructions. RNA was quantified with a NanoDrop One Spectrophotometer (ThermoFisher). cDNA 
was synthesized using $1 \mu \mathrm{g}$ RNA with SuperScript IV reverse transcriptase (Invitrogen) in a $20 \mu \mathrm{L}$ volume. qPCR was performed using Fast SYBR Green Master Mix (ThermoFisher) with primers obtained from Integrated DNA Technologies (IDT) (Table 9). Reactions were run on the StepOnePlus Real-Time PCR System (ThermoFisher). Raw data was quantified in Microsoft Excel and statistics performed in GraphPad's Prism 8 (La Jolla, CA). Polymerase chain reaction (PCR) was carried out for spliced-XBPI and XBPI expression with PCR Supermix (Invitrogen). Amplicons were evaluated via 2\% agarose gels (Bio-Rad). $10 \mu \mathrm{L}$ of amplicons were added to $2 \mu \mathrm{L} 6 \mathrm{X}$ Loading Buffer (Invitrogen). Gels were run at $80 \mathrm{~V}$ for 60 minutes and imaged via ChemiDoc Imaging System with Image Lab Touch Software (Bio-Rad, Hercules, CA).

Western Blot. The iPSC or HLC were treated as described above. Following treatment, the cells were thoroughly washed with $\mathrm{PBS}^{+/+}$prior to adding $200 \mu \mathrm{L}$ RIPA lysis buffer (ThermoFisher) plus protease inhibitor cocktail (ThermoFisher) for cell collection. Lysates were rocked overnight at $4^{\circ} \mathrm{C}$, followed by centrifugation for 15 minutes. Supernatants were used for protein quantification by DC Protein Assay (Bio-Rad). 10 $\mu \mathrm{g} / \mathrm{sample}$ of total protein was run on a 4-10\% mini-protean TGX precast gels (Bio-Rad) at 200 volts for 40 minutes. Proteins were transferred onto PVDF (Bio-Rad) then blocked in 3\% milk/PBST. Membranes were probed overnight with the LDLR antibody (1:1000 in 5\% BSA/PBST; R\&D Systems) or $\beta$-actin (1:1000; Santa Cruz Biotechnology, Dallas, TX) overnight at $4^{\circ} \mathrm{C}$. The membranes were incubated in either HRP-Bovine anti-goat $\operatorname{IgG~H}+\mathrm{L}$ (Jackson ImmunoResearch, West Grove, PA) or anti-mouse IgG (Cell Signaling, Danvers, MA), HRP-linked antibodies (1:5000 in 3\% milk/PBST) the following day for 1 hour at room temperature. Clarity Max Western ECL Blotting Substrate (Bio-Rad) was used to 
visualize the proteins on the Bio-Rad Imager. Densitometry was performed using Bio-Rad imaging software. A list of all antibodies used for analyses is presented in Table $\mathbf{1 0 .}$

Cellular Cholesterol Replenishment. Cells were plated on $35 \mathrm{~mm}$ tissue culture dishes and differentiated until day one of stage three, as described above. Cells were treated with LPDS media supplemented with $5 \mu \mathrm{M}$ Rosuvastatin for 48 hours. After 48 hours, cells were treated with LPDS media supplemented with $5 \mu \mathrm{M}$ Rosuvastatin and $10 \mathrm{mM}$ methyl$\beta$-cyclodextrin (Sigma-Aldrich) for 45 minutes [309]. After 45 minutes (timepoint 0-hour), cells were collected as described below. The remaining dishes were cultured with LPDS media supplemented with $5 \mu \mathrm{M}$ Rosuvastatin and $10 \mu \mathrm{g} / \mathrm{mL}$ LDL-C (ThermoFisher). Cells were collected at 6 and 24 hours.

Cells were collected by incubating in TrypLE Express (ThemoFisher) for five minutes then gently scraping and transferring cells to a $15 \mathrm{~mL}$ tube. After centrifugation (200 x $g$, 4 minutes), the cell pellet was re-suspended in $200 \mu \mathrm{L}$ chloroform/methanol (2:1 v/v) mixture, vortexed, and centrifuged (14,000 x $g, 5$ minutes) to allow separation into three layers. A micropipette was used to carefully discard the top aqueous layer containing RNA. Next, a micropipette was used to gently push past the interphase layer (a thin membrane of protein) to reach the organic phase layer containing the lipids. This bottom lipid layer was transferred into a new microcentrifuge tube. The lipid solution was dried using the Savant SpeedVac Plus vacuum (ThermoFisher) for 30 minutes. The dried lipids were re-suspended in $1 \mathrm{X}$ reaction buffer supplied in the Amplex Red Cholesterol Assay kit (ThermoFisher). The middle protein layer was re-suspended in RIPA lysis buffer, incubated overnight at $4^{\circ} \mathrm{C}$, and processed the day next day for protein analysis. 
Collected lipid content was analyzed with the Amplex Red Cholesterol Assay Kit per the instructions. Readings were measured on the Synergy4 spectrophotometer (BioTek, Winooski, VT) with Gen5 software (BioTek) at an excitation of $560 \mathrm{~nm}$ and emission detection at $590 \mathrm{~nm}$. Lipid content was normalized to total protein level as measured by the DC protein assay.

Fluorescence-labeled LDL uptake assay. NC-iPSC and C-iPSC were plated in 3 of 4 wells of a 4-well chamber slide and differentiated to HLC (see above). Three wells were treated overnight in LPDS media supplemented with $5 \mu \mathrm{M}$ Rosuvastatin. The following day, two wells in Rosuvastatin were treated with $10 \mu \mathrm{g} / \mathrm{mL}$ low density lipoprotein labeled with 1,1'-dioctadecyl-3,3,3',3'-tetramethyl-indocarbocyanine perchlorate (fluorescently labeled DiI-LDL) (ThermoFisher) for either 6 or 24 hours while the remaining well did not receive any DiI-LDL. Cells were fixed with 2\% PFA/PBS (10 minutes, $24^{\circ}$ C; Electron Microscopy Sciences, Hatfield, PA) and mounted with VECTASHIELD Antifade Mounting Medium with DAPI (Vector Laboratories, Burlingame, CA). Slides were imaged using Olympus IX81 fluorescence microscope (Center Valley, PA) at 20X magnification.

Immunocytochemistry and Image Analysis. Following Rosuvastatin treatment overnight in LPDS media, HLC were fixed with $2 \%$ PFA/PBS (10 minutes, $24^{\circ} \mathrm{C}$ ), permeabilized with $0.05 \%$ Triton X-100/PBS (10 minutes, $24^{\circ}$ C; Sigma Aldrich) and washed with PBS. Cells were then blocked with 5\% normal donkey serum/PBS (Jackson ImmunoResearch) for 1 hour followed by blocking with an avidin/biotin blocking kit (Vector Laboratories). Primary antibodies were diluted in 5\% donkey serum/PBS and incubated on cells overnight at $4^{\circ}$ C. Secondary antibodies diluted in 5\% donkey 
serum/PBS (1:1000) were added to the cells (2-hour, $24^{\circ} \mathrm{C}$ ) followed by washing and mounting with VECTASHIELD Antifade Mounting Medium with DAPI. Slides were imaged using Olympus BX61WI confocal microscope with Fluoview (FV10-ASW 4.1, Olympus). The four channels were merged into a single image using AMIRA software (ThermoFisher) [310]. Using Fluoview (FV10-ASW 4.1), confocal image stacks were analyzed utilizing the colocalization processing tool to both visualize overlapping of images as well as quantification of overlap. A list of all antibodies used for analyses is listed in Table 10.

Statistical Analysis. Data from three independent experiments were analyzed via one or two-way ANOVA with a post-hoc using Tukey multiple comparison test in GraphPad Prism 8 and expressed as mean \pm SEM. Figure legends contain further details.

\section{Table 9}

$\underline{\text { PCR Primers for LDLR and ER Stress }}$

\begin{tabular}{|c|c|c|c|}
\hline Gene & Primer 1 (5' $\rightarrow$ 3') & Primer 2 (5' $\rightarrow$ 3') & Product Size (bp) \\
\hline LDLR & GCAGTGTGACCGGGAATATGA & GTTGGTCCCGCACTCTTTGA & 115 \\
\hline Grp78 & CCGTTCAAGGTGGTTGAAAAGAA & TGGCGTTGGGCATCATTAAAA & 200 \\
\hline (S) XBP1 & CCTGGTTGCTGAAGAGGAGG & GGCAGGCTGCTGTCCTCAT & $150 ; 124$ \\
\hline P0 & TCGACAATGGCAGCATCTAC & ATCCGTCTCCACAGACAAGG & 200 \\
\hline
\end{tabular}




\section{Table 10}

List of Antibodies and Fluorophores for WB and ICC

\begin{tabular}{|l|l|l|l|}
\hline Antigen & Host & Dilution & Distributor \\
\hline LDLR & Goat & $\begin{array}{l}1: 100 \text { (ICC), 1:1000 } \\
\text { (WB) }\end{array}$ & R\&D Systems, Minneapolis, MN \\
\hline Calnexin & Mouse & $1: 100$ & EMD Millipore, Billerica, MA \\
\hline $\begin{array}{l}\text { Biotinylated-Wheat } \\
\text { Germ Agglutinin }\end{array}$ & - & $1: 200$ & Vector Laboratories, Burlingame, CA \\
\hline Actin & Mouse & $1: 1000$ & Santa Cruz Biotechnology, Dallas, TX \\
\hline gIgG & Goat & $\mathbf{1 : 1 0 0}$ & Novus Biologicals, Littleton, CO \\
\hline mIgG2b & Mouse & $\mathbf{1 : 1 0 0}$ & Invitrogen, Carlsbad, CA \\
\hline $\begin{array}{l}\text { Alexa Fluor Donkey } \\
\text { Anti-Goat 488nm }\end{array}$ & $1: 1000$ & Invitrogen \\
\hline $\begin{array}{l}\text { Alexa Fluor Donkey } \\
\text { Anti-Mouse 546nm }\end{array}$ & $1: 1000$ & Invitrogen \\
\hline $\begin{array}{l}\text { Streptavidin-649 } \\
\text { HRP-Bovine Anti-Goat } \\
\text { IgG H+L }\end{array}$ & $1: 1000$ & Vector Laboratories \\
\hline $\begin{array}{l}\text { Anti-Mouse IgG, HRP- } \\
\text { linked }\end{array}$ & $1: 5000$ & $\begin{array}{l}\text { Jackson ImmunoResearch, West Grove, } \\
\text { PA }\end{array}$ \\
\hline
\end{tabular}

\section{RESULTS}

\section{$\underline{\text { LDL-C Internalization is Restored in Corrected Hepatocyte-Like Cells }}$}

Our previously published work was focused on iPSC and the restoration of LDL-C internalization in FH-iPSC using CRISPR/Cas9 [189]. This is potentially important for studying the effects of statins on pregnant FH patients. The hepatocyte is responsible for cholesterol regulation and is the cell making up the liver parenchyma. After CRISPR/Cas9 correction of cells, we saw an internalization of LDL-C was restored in HLC [189]. We wanted to examine a time course for replenishment of LDL-C and whether or not the NCHLC could recover over a 24-hour period by non-receptor mediated mechanisms. We treated HLC overnight in LPDS media with Rosuvastatin (0h), then added $10 \mu \mathrm{g} / \mathrm{ml}$ DiILDL for 6 (6h) or 24 (24h) hours. As expected at the 0h, no DiI fluorescence was detected in either NC or C-HLC. After 6h, NC-HLC still had not internalized a detectable level of 
DiI-LDL as evidenced by lack of fluorescence signal. After 24 hours, a minimal DiI fluorescence signal was detected suggesting internalization by non-receptor mediated mechanism or through the expected 5\% LDLR activity of this mutation (Figure 21A, top panels). In contrast, the C-HLC presented a far greater capacity for DiI-LDL internalization over this time frame (Figure 21A, bottom panels). At $6 \mathrm{~h}$, the C-HLC showed bright DiI fluorescence that qualitatively increased over 24h (Figure 21A). This indicates that the corrected cells are capable of internalizing LDL via receptor mediated mechanisms and that statin treatment produces a time dependent LDL internalization increase.

We next asked what is the ability of NC and C-HLC to restore cellular cholesterol after depletion with methyl- $\beta$-cyclodextrin. HLC were incubated in LPDS media supplemented with Rosuvastatin overnight for 48 hours as described, then treated with 10 $\mathrm{mM}$ methyl- $\beta$-cyclodextrin for 45 minutes to extract any cholesterol stored within the cell [309]. Samples collected after 45 minutes and designated as time point zero or basal cholesterol levels. At t0, unlabeled LDL-C was added to the remaining cells for 6 and 24 hours after which samples were also collected. Cholesterol content was measured using Amplex Red quantification [311] and normalized to total protein (Figure 21B). At t0 after cyclodextrin treatment, the starting cellular cholesterol content for both NC and C-HLC were 15.7 and $11.6 \mu \mathrm{M} /(\mu \mathrm{g} / \mu \mathrm{L})$ with no statistical difference, indicating the starting cholesterol content was equivalent for both cell populations. Six hours after cyclodextrin treatment the NC and C-HLC cholesterol content was 10.6 and 14.5, respectively, which was still not significantly different between the cell groups at this time point nor different from the $\mathrm{t} 0$ starting time point. It was only after $24 \mathrm{~h}$ that a statistically significant difference 
was quantified. At 24h, the NC-HLC contained 21 cholesterol/unit protein, which statistically was equivalent to t0 and t6h. When the LDLR is corrected, C-HLC contained statistically significant $(p<0.05)$ increase in cellular cholesterol of 61 with treatment compared to t0 and t6h as well as NC-HLC. This demonstrates a quantitative increase in the corrected HLC's ability to internalize cholesterol and the receptor corrected cells have the ability to overcome cyclodextrin inhibited endocytosis [312]. Together this data supports the normalization of LDLR mediated LDL-C endocytosis in FH differentiated HLC when the class II mutation is directly corrected. 


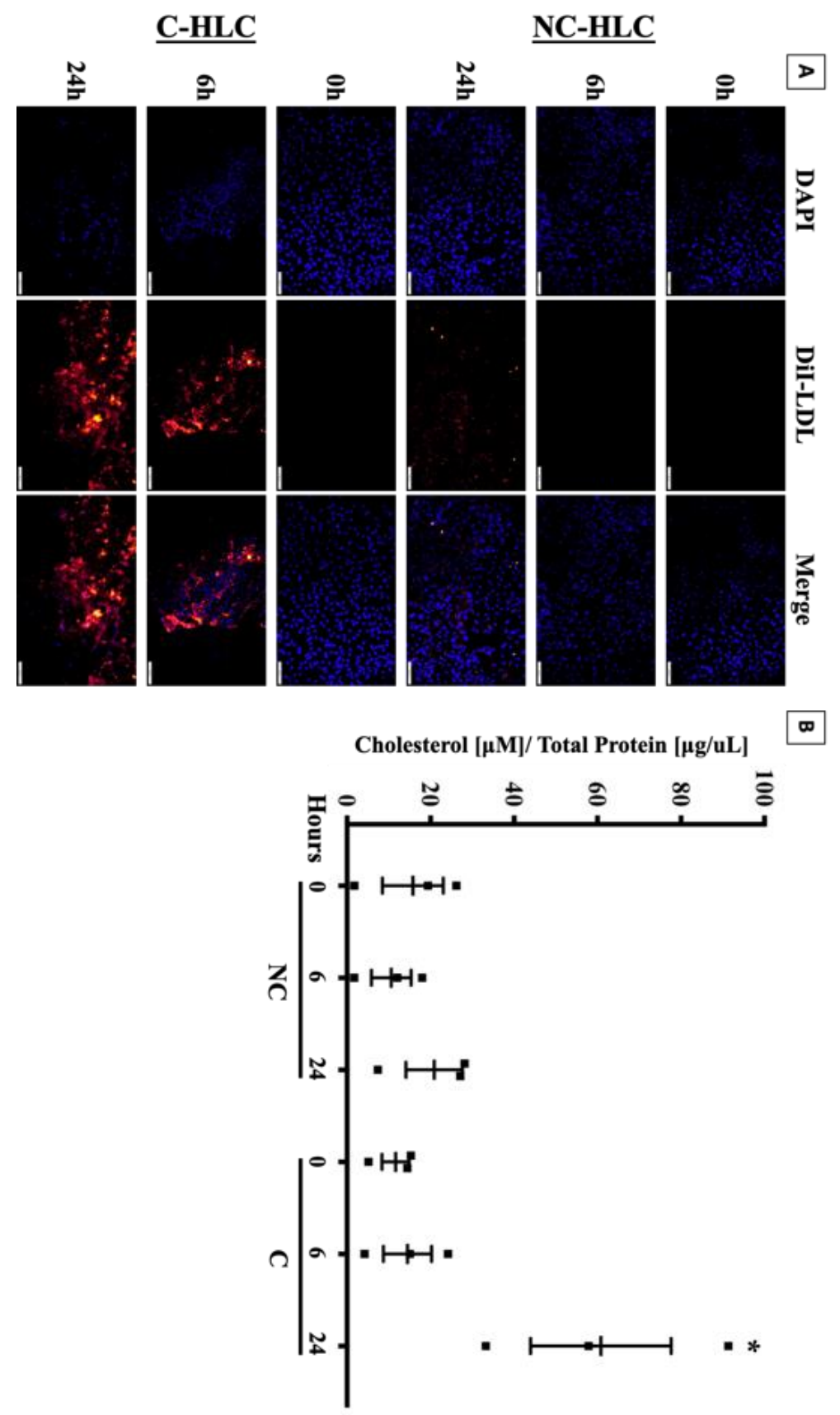

Figure 21. LDLR-Mediated Cholesterol Internalization is Restored in C-HLC. (A) NCand C-HLC were treated overnight in 5\% lipoprotein deficient serum media (LPDS) supplemented with Rosuvastatin. This was followed by incubation with DiI-LDL for $6 \mathrm{~h}$ or 24h. NC-HLC did not show any DiI fluorescence from LDL uptake until 24h. C-HLC internalized DiI-LDL by $6 \mathrm{~h}$, which increased after $24 \mathrm{~h}$. Scale bars $=100 \mu \mathrm{m}$ (B) HLC were treated with LPDS media and Rosuvastatin for $48 \mathrm{~h}$ followed by incubation in methyl- $\beta$ cyclodextrin (MBC) for 45 minutes when time point $0 \mathrm{~h}$ samples were collected. Cells were further incubated for $6 \mathrm{~h}$ or $24 \mathrm{~h}$ with unlabeled LDL then analyzed for cellular cholesterol concentration with respect to protein. NC-HLC showed no statistical difference in cellular 
cholesterol for all three time points demonstrating the dysregulation of cholesterol metabolism in FH HLC. Corrected cells did not have statistically different cholesterol content after $6 \mathrm{~h}$ demonstrating the effect of MBC treatment and plasma membrane cholesterol chelation on receptor-medicated endocytosis. After 24h exposure to LDL-C, receptor corrected HLC were able to increase cellular cholesterol to a statistically significant level $(61 \mu \mathrm{M} /(\mu \mathrm{g} / \mu \mathrm{L})$ compared to t0 $11.6 \mu \mathrm{M} /(\mu \mathrm{g} / \mu \mathrm{L})$. There was no difference in cholesterol concentration at t0 between NC- and C-HLC. The graph values represent the mean \pm SEM $(n=3)$ per treatment, per cell type. Statistics were performed using a two-way ANOVA Tukey post-hoc test. *p $<0.05$ compared to NC-HLC $6 \mathrm{~h}, 24 \mathrm{~h}$ and C-HLC 6h.

\section{Distribution of LDLR in FH and LDLR Corrected HLC}

The normal LDLR is synthesized in the ER as a partially glycosylated precursor of 120-kDa. Upon reaching the Golgi, N-and O-linked sugars are processed for a molecular weight of $160-\mathrm{kDa}$. This process is delayed or completely abolished in class II mutations, trapping the misfolded LDLR in the ER [92]. Previous work using fibroblasts containing the same homozygous $3 \mathrm{bp}$ deletion in the LDLR as our patient FH cells were studied through electron microscopy to identify the distribution of the intracellular LDLR [118]. Unlike normal fibroblasts where most all LDLR was found in coated pits, the class II mutant cells had less than 5\% of LDLR detectable on the cell surface, in coated pits or vesicles in the endocytic pathway. Instead, most of the class II LDLR was present in membrane extensions of the rough ER [118]. We sought to examine if NC-HLC retained the LDLR in the ER and if correction allowed proper movement of the LDLR to the membrane.

Situated within the ER is calcium-binding Calnexin [313]. Calnexin interacts with newly synthesized proteins, acting to retain misfolded proteins in the ER [313]. Wheat germ agglutinin (WGA) is a lectin that binds to $\mathrm{N}$-acetylglucosamine found in the plasma

membrane of different cell types including hepatocytes [314, 315]. After differentiation, 
HLC were treated overnight in LPDS and Rosuvastatin after which we examined protein localization using immunocytochemistry for Calnexin, LDLR, and WGA. Stacking of images into a 3D rendering presented the appearance of class II LDLR localizing with ERCalnexin in NC-HLC (Figure 22A). Using Fluoview Program software, images were further analyzed utilizing the colocalization processing tool to both visualize overlapping of images as well as quantification of overlap. In Figure 22B, a slice from a 3D stack is presented. To aid in visualization, for NC vs. C-HLC, we focused on two channels at a time and pseudo-colored Calnexin or WGA red so that colocalization with LDLR (Green) will display as yellow. NC-HLC detection of the class II LDLR shows a compact localization that when combined with Calnexin, illustrates colocalization in the ER (top row, left panel). When NC-HLC LDLR is combined with plasma membrane WGA (bottom row, left panel), no colocalization as represented by yellow is detected. In contrast, C-HLC present a smaller amount of merging of LDLR with ER-Calnexin (Figure 22B, top row, right panel) while demonstrating a significant colocalization of LDLR with WGA-plasma membrane (bottom row, right panel). Quantification of overlap confirmed NC-HLC have almost twice the amount of LDLR colocalizing to the ER-Calnexin (0.34) than WGAplasma membrane (0.16) and the difference was statistically significant by one-way ANOVA with Tukey post-hoc test $(p<0.05)$ (Figure 22C). In C-HLC, there was significant variability between samples and though a slight increase in WGA-plasma membrane and LDLR colocalization compared to ER-Calnexin was calculated, this was not significant (Figure 22C). This data confirms that NC-HLC retains the LDLR in the ER, but in spite of other evidence clearly demonstrating a function LDLR at the plasma 
membrane surface, we were unable to quantify a statistically significant change in localization.

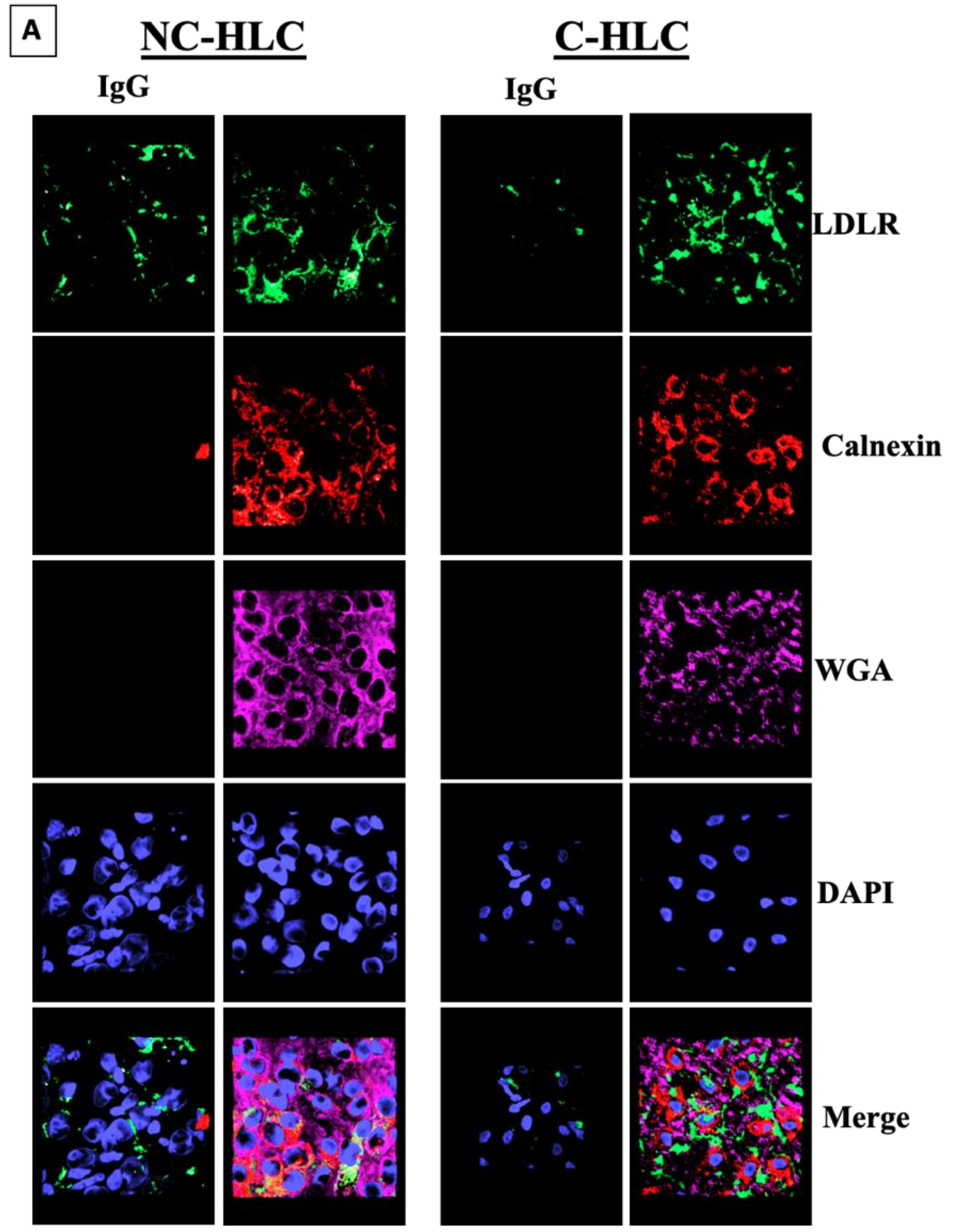




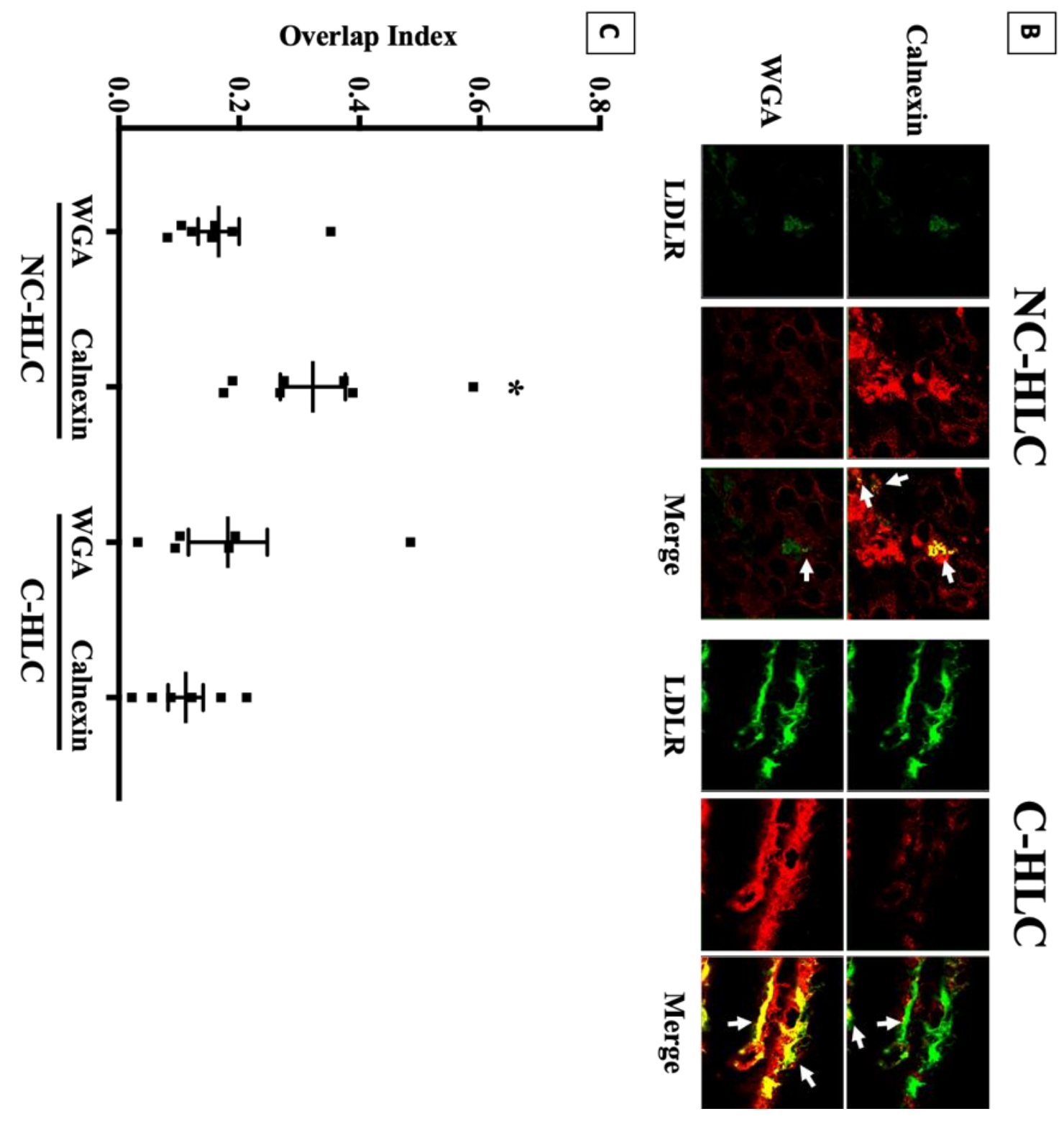

Figure 22. LDLR Colocalizes with Calnexin-ER in FH-HLC. (A) After differentiation, HLC were treated overnight in LPDS media supplemented with Rosuvastatin followed by ICC and confocal imaging, 100x oil immersion objective. AMIRA software was used to stack slices and merge channels to present an overview of localization of the LDLR (green) Calnexin-ER (red), WGA-plasma membrane (magenta), and nucleus (DAPI). (B) Fluoview software was used to visualize overlap between the LDLR-Calnexin-ER or LDLR-WGA-plasma membrane. The channel for WGA-plasma membrane was changed to red for visualization on overlap. Overlap is shown in yellow. NC-HLC appear to have a greater amount of yellow with the LDLR-Calnexin (left, top row) compared to LDLRWGA (left, bottom row). C-HLC appear to have an increase in colocalization of LDLRWGA (right, bottom row) compared to LDLR-Calnexin (right, top row). (C) Quantification of colocalization using Fluoview software indicated that NC-HLC did have a significantly greater colocalization of the LDLR with Calnexin than WGA. C-HLC showed a slight 
increase in LDLR-WGA colocalization compared to LDLR-Calnexin though it was not significant. The graph values represent the overlap index \pm SEM $(n=5)$ per cell type. Statistics were performed using a one-way ANOVA Tukey post-hoc test. ${ }^{*} p<0.05$ of NCHLC LDLR-Calnexin to NC-HLC WGA, C-HLC WGA, and C-HLC Calnexin.

\section{$\underline{\text { Rosuvastatin Upregulates LDLR Transcript and Protein Levels in FH and Corrected Cells }}$}

In our previously published work, we used Lovastatin in our studies and found that NC-iPSC expressed immature LDLR compared to C-iPSC that converted its protein to all mature LDLR [189]. We confirmed this once more in our iPSC cells after treatment with Lovastatin in LPDS media. Lovastatin did increase LDLR protein expression in all 3 cell types (Figure 23A), and the NC-iPSC presented both the mature and immature form of LDLR similar to control recombinant human LDLR (rhLDLR). Lovastatin inhibits HMGCoA reductase, preventing cholesterol synthesis leading to an upregulation of LDLR. In addition, Lovastatin has been cited to inhibit or modulate the $20 \mathrm{~S}$ proteasome pathway [316-318]. The LDLR receptor class II mutants have been reported to be degraded via the $26 \mathrm{~S}$ proteasome-pathway [119]. To ensure the accumulation of immature LDLR that we observed was not due to effects on the proteasomal pathway, we opted to use a contemporary drug, Rosuvastatin.

To confirm the form of LDLR protein in NC, C, and control H1 stem cells, we treated the iPSC with Rosuvastatin or excess sterols in LPDS media overnight. Excess sterols act independently of the LDLR and enter the cell via pinocytosis to decrease LDLR expression. NC-iPSC demonstrated a major accumulation of the immature LDLR not seen in the C-iPSC or H1-ESC (Figure 23A), suggesting the CRISPR modification restored the normal LDLR structure allowing LDLR to proceed to the Golgi for processing and movement to the plasma membrane. Quantification and of total LDLR indicated a 
significantly greater total LDLR in NC-iPSC than in C-iPSC and H1-ESC (Figure 23B). Two-way ANOVA with a Tukey post-hoc test determined significance of total LDLR in all 3 iPSC/ESC lines treated with Rosuvastatin compared to excess sterols $(* * * p<0.001$, **** $p<0.0001)$ and NC-iPSC Rosuvastatin compared to C-iPSC and H1-ESC with Rosuvastatin $(\# p<0.001)$.

We differentiated the iPSC/ESC to HLC, and after treatment with Rosuvastatin, we observed an induction of LDLR expression primarily in immature form in NC-HLC and very little mature LDLR (Figure 23C). C-HLC and H1-HLC expressed solely mature LDLR (Figure 23C). Excess sterols downregulated LDLR protein synthesis in all the cells as expected. Quantification and two-way ANOVA with a Tukey post-hoc test confirmed an upregulation of total LDLR protein mainly in NC-HLC compared to NC-HLC in excess sterols and H1-HLC in Rosuvastatin $(* * p<0.01$, $\# p<0.01)$ (Figure 23D).

To investigate if total LDLR protein and increase in immature LDLR (Figure 23B, D) was due to differential transcriptional regulation of the LDLR between NC and C cells in the presence of statins, qPCR analysis was used on both iPSC and HLC post-treatment with control carrier DMSO, Rosuvastatin, and excess sterols. For both iPSC and differentiated HLC, Rosuvastatin increased LDLR transcript levels across all the cell types compared to excess sterols or DMSO control (Figure 23E, F), which two-way ANOVA with a Tukey post-hoc test showed to be significant $(* * p<0.01, * * * * p<0.0001)$. However, there was no difference in transcript levels when comparing statin treatment across cell lines or differentiation state, which is to be expected with statin treatment.

Taken together, this shows that both iPSC and HLC can be induced to express LDLR with statin treatment and while the NC generally has more total LDLR that 
accumulates predominately in the ER, this is not due to any difference in transcription. This suggests the accumulating misfolded LDLR may be due to an issue with protein ER processing or degradation. 

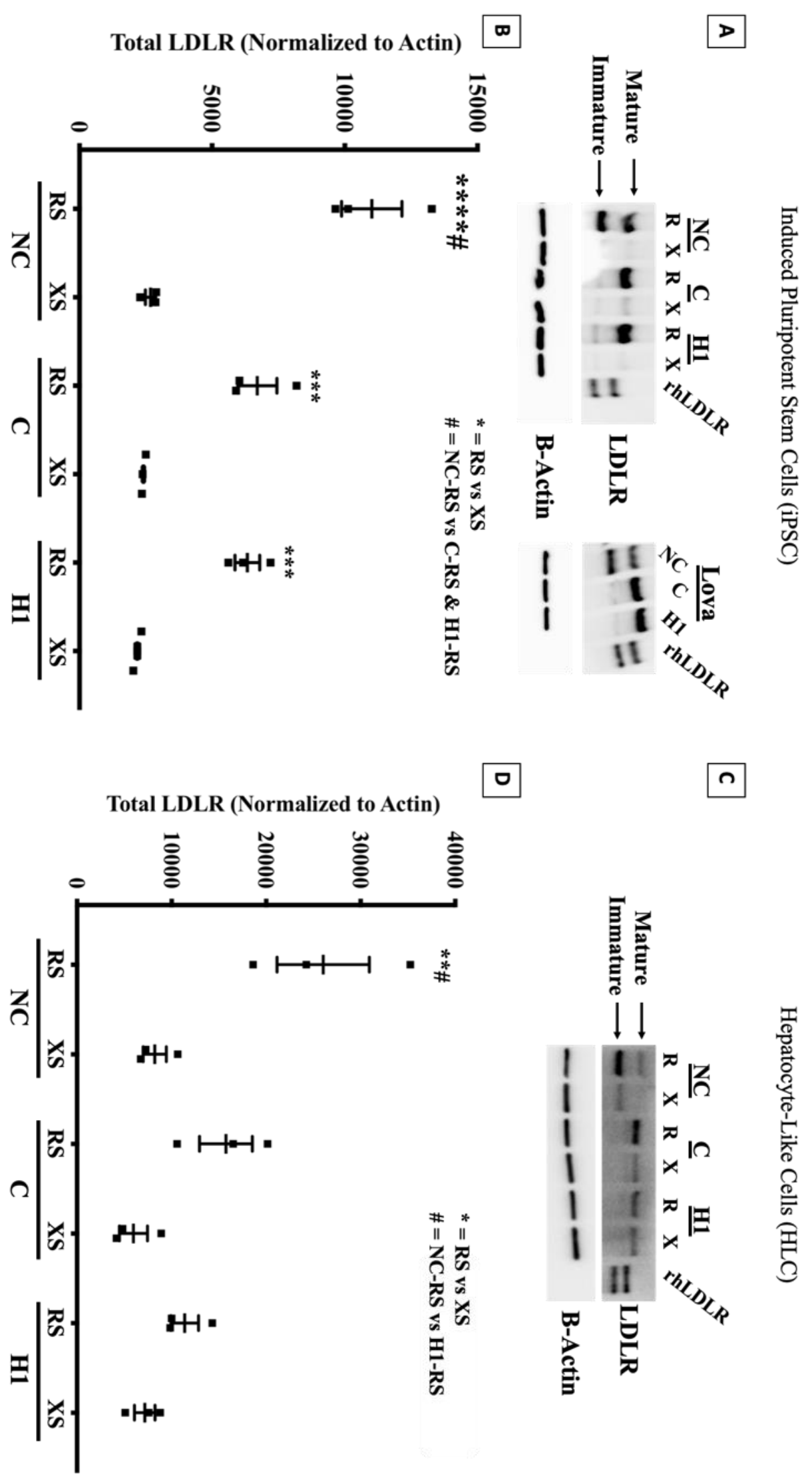

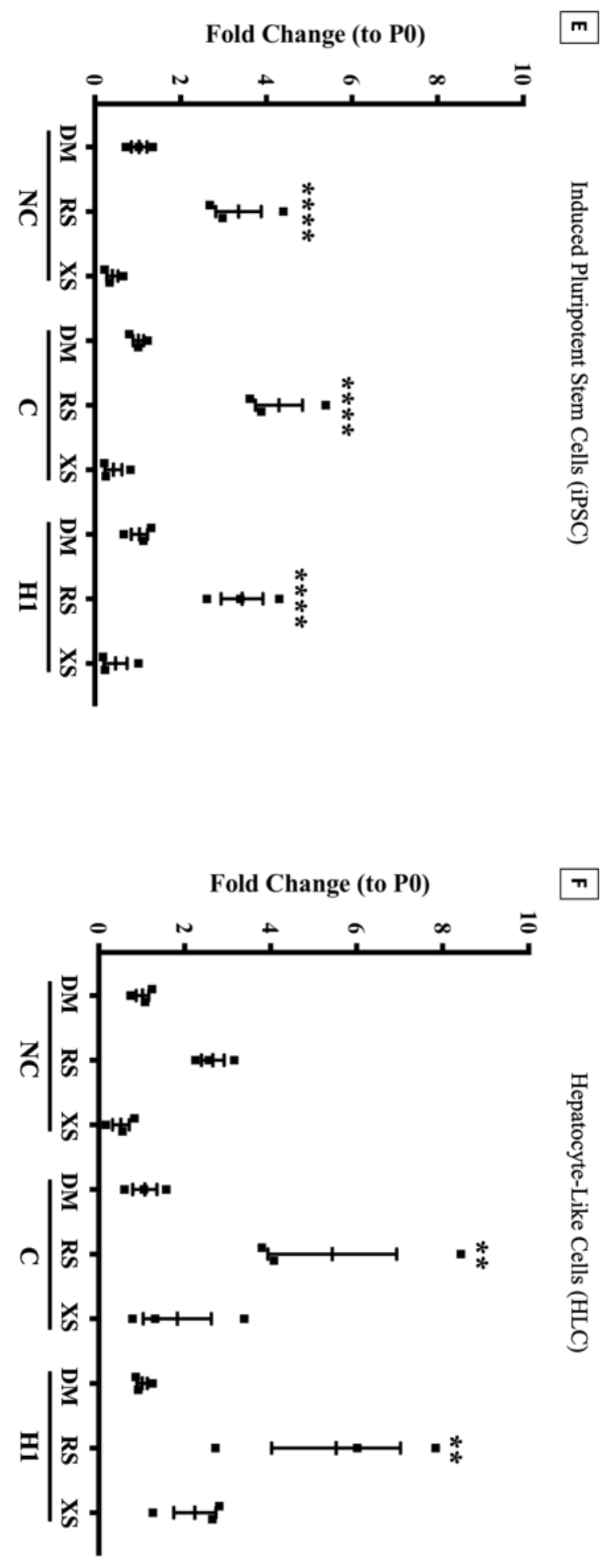
Figure 23. Rosuvastatin Increases Total LDLR Protein Levels and an Accumulation of Immature LDLR in NC-cells. (A) iPSC were treated overnight in LPDS media supplemented with either Lovastatin (Lova), Rosuvastatin (RS), or excess sterols (XS). Western blot analysis for LDLR shows NC-iPSC upregulate immature LDLR in Lovastatin while C-iPSC and H1-ESC express mature LDLR. Rosuvastatin treatment shows the same response and LDLR is suppressed when exposed to sterols. Recombinant human LDLR protein (rhLDLR) was used as detection control. (B) Quantification of total LDLR demonstrated that statin-treated-NC-iPSC had a significantly greater total LDLR than CiPSC and H1-ESC treated with Rosuvastatin. All 3 statin-treated cell types were significantly greater compared to their excess sterol treated counterparts. (C) iPSC/ESC were differentiated to HLC. Under the same conditions of RS or XS treatment, NC-HLC express greater total LDLR, predominately as immature protein, that is converted to all mature in C-HLC and H1-HLC. LDLR levels decreased with sterols treatment. (D) Quantification of total LDLR presented a significant increase in total LDLR in statintreated-NC-HLC compared to its excess sterols. In addition, statin-treated-NC-HLC had a significantly larger amount of LDLR compared to statin-treated H1-HLC. (E) qPCR analysis of LDLR shows Rosuvastatin (RS) treatment significantly increases LDLR transcript levels statistically equivalently in NC-and C- iPSC like that of H1-ESC compared to DMSO (DM) control and excess sterol (XS) treatment. (F) Similarly, HLC showed consistent results with significantly upregulated LDLR mRNA levels with RS treatment and no difference between cell type. The graph values represent the mean \pm SEM $(\mathrm{n}=3)$ per treatment, per cell type using a two-way ANOVA Tukey post-hoc test. $* * \mathrm{p}<0.01, * * * \mathrm{p}<0.001, * * * * \mathrm{p}<0.0001 \# \mathrm{p}<0.05$ between RS-treated cell type

\section{$\underline{\text { Treatment of Cells with Rosuvastatin Does Not Induce ER Stress }}$}

Two studies reported that class II LDLR mutants are retained in the ER causing ER stress and activating the UPR. Since statins cause an accumulation of immature protein in NC FH-iPSC and HLC, we investigated statin treatment induced ER stress in class II iPSC/HLC and if it was normalized in the corrected cells. ER chaperone Grp78 is a major factor involved in maintaining ER homeostasis. It is also the first component activated in the UPR. Little is known about the ER stress responses in iPSC or derived HLC, therefore, we used the ER stress inducer, tunicamycin as a positive control. Tunicamycin functions by inhibiting glycoprotein synthesis, inducing protein unfolding and activating the UPR. iPSC/HLC were treated with either Rosuvastatin, excess sterols, and control DMSO overnight or tunicamycin for 4.5 hours. We specifically looked at Grp78 mRNA transcript 
levels since it has been documented that Grp78 mRNA levels increase quickly in responses to ER stress. In addition, in an overexpression class II LDLR mutant model, Grp78 mRNA and protein levels are upregulated [124, 125]. After treatment with Rosuvastatin, both iPSC and HLC did not produce any quantifiable increase in Grp78 expression in any of the cell types (Figure 24A, B). There was no difference in transcripts between statin treatment and control DMSO or excess sterols. However, tunicamycin significantly increased Grp78 transcript levels across all cell types in both iPSC and HLC demonstrating that UPR pathways were capable of being activated (Figure 24A, B). Two-way ANOVA with a posthoc Tukey test confirmed a significant upregulation of Grp78 mRNA with tunicamycin treatment $(* * * p<0.001, * * * * p<0.0001)$.

$\mathrm{X}$-box binding protein 1 (XBP1) is a transcription factor that becomes activated in response to accumulation of unfolded proteins [218]. The splicing of a 26-nucletoide intron from XBPI generates the transcription factor, spliced XBP1 (SXBP1) [213, 221, 223]. SXBP1 regulates UPR genes for folding, ERAD, autophagy, and organelle biogenesis [213, 224-226]. The SXBP1 isoform can be detected through PCR and after tunicamycin treatment, our cells exhibited the SXBP1 isoform (Figure 24C, D) in the iPSC/ESC or HLC. However, no XBP1 splicing was detected with any other treatment in the NC or C iPSC and HLC. Though H1 hESC did not show XPB1 splicing other than Tunicamycin, curiously H1 HLC had a basal level of SXBP1 which was statistically equivalent to Tunicamycin treatment. Quantification of SXBP1 to total XBP1 confirmed that Rosuvastatin treatment did not activate SXBP1 or the UPR in NC, C, or H1 cells (Figure 24C, D). Two-way ANOVA with a Tukey post-hoc test showed that only tunicamycin 
treatment significantly activated the splicing of XBP1 in all iPSC/ESC $(* * * * p<0.0001)$ (Figure 24C). No significance was found in HLC treated with tunicamycin.

We further evaluated the activation of the UPR through western blot. During ER stress, ATF6 is cleaved and releases a 50-kDa fragment termed ATF6f that continues on to act as a transcription factor and upregulate expression of UPR genes involved in protein folding and degradation [221, 232]. CHOP is a protein downstream of the PERK pathway. Its activation signifies the cell's response towards an apoptotic state due to an overwhelming amount of ER stress. We ran a western blot for ATF6, CHOP, and Grp78. Across all HLC, Rosuvastatin and excess sterol treatment did not change the levels of ATF6 (Figure 24E). HLC treated with tunicamycin had a decrease in expression of the ATF6 protein; however, we could not detect the ATF6f (Figure 24E). The expression pattern of decreased ATF6 with only tunicamycin was indicative that this was specific to some sort of stress response that was not present with statin or excess sterol treatment. CHOP was not present until HLC were treated with tunicamycin in NC, C, and H1 (Figure 24E). Grp78 levels were upregulated in all HLC treated with tunicamycin (Figure 24E) correlating with qPCR data (Figure 24B). Altogether, this data shows that although FH $\mathrm{NC}$ and $\mathrm{C}$ cells as well as normal $\mathrm{H} 1$ controls can be induced to activate ER stress and UPR with Tunicamycin treatment, exposure to statins that cause downstream LDLR upregulation does not induce ER stress, as has been shown by others. 

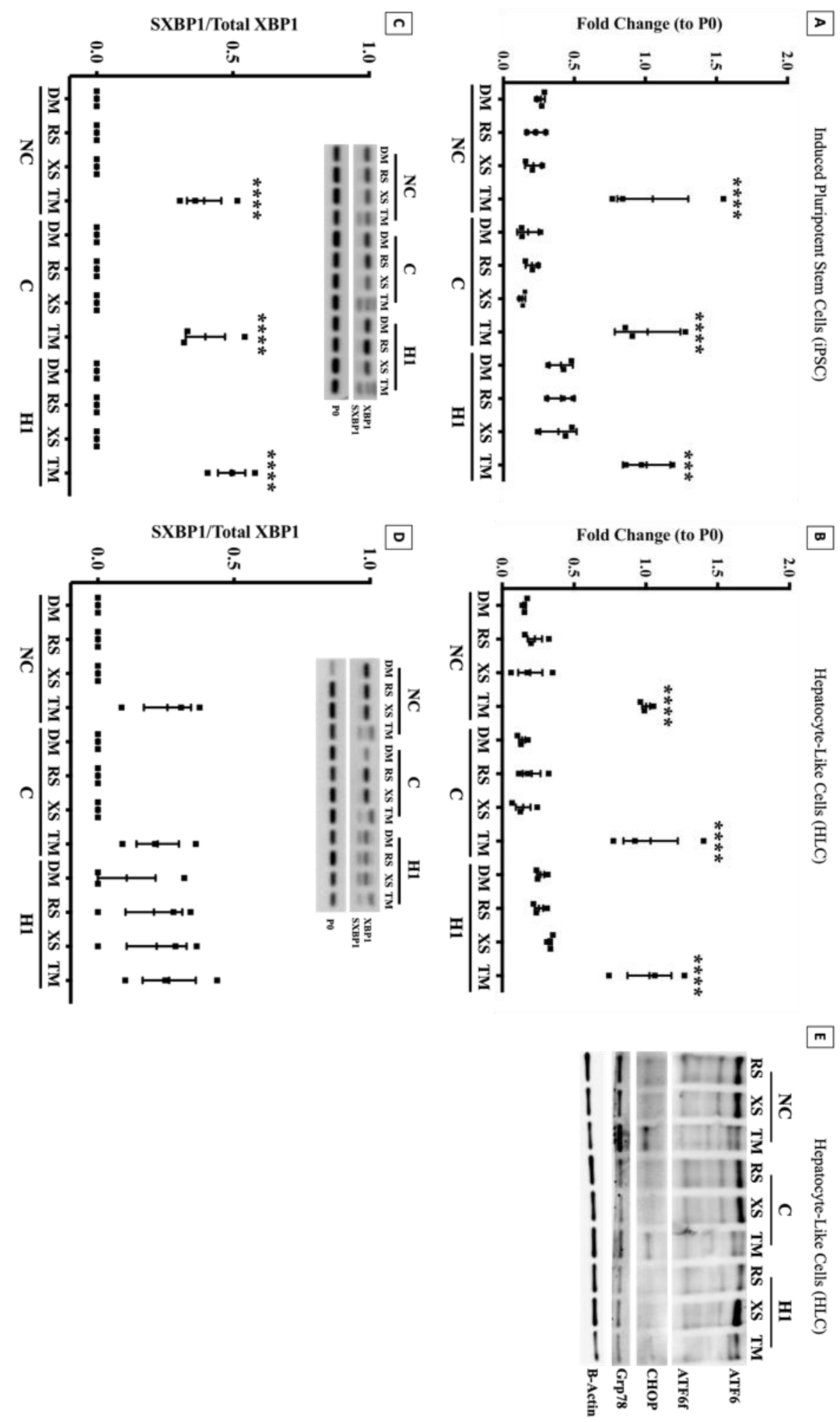
Figure 24. FH-NC Cells Do Not activate the UPR Following Rosuvastatin Treatment. Following treatment with either carrier DM, RS, XS, or tunicamycin (TM; $5 \mu \mathrm{g} / \mathrm{mL}$ for 4.5 hours), cells were tested for ER stress and UPR markers at both mRNA and protein levels in iPSC/ESC and HLC. (A) qPCR analysis in iPSC indicated Grp78 mRNA levels do not change with RS treatment compared to DM/XS controls across the 3 cell groups. TM significantly increased Grp78 mRNA in NC-, C-, and H1 stem cells. (B) HLC presented the same trend with TM significantly upregulating Grp78 transcripts while RS did not. (C) PCR for spliced-XBP1 (SXBP1) visualized on a 2\% agarose gel indicated TM treatment induced splicing of XBP1 that was not present in RS treated iPS/ES cells. Quantification of SXBP1/XBP1 shows significant SXBP1/XBP1 activation in TM-treated cells across all 3 cell types. (D) HLC presented similarly to iPSC/ESC for SXBP1/XBP1. Quantification of SXBP1/XBP1 did not show any significance. (E) Western blot analysis showed a decrease in ATF6 levels in TM-treated HLC only while RS- and XS-treated HLC did not change. CHOP was expressed only in TM-expressed HLC. Grp78 protein levels were upregulated in TM-treated HLC while staying closer to basal level in RS-and-XS-treated HLC. The graph values represent the mean \pm SEM $(n=3)$ per treatment, per cell type using a two-way ANOVA Tukey post-hoc test. ****p<0.0001 of TM-treatment to all other treatments in each respected cell group.

\section{$\underline{\text { DISCUSSION }}$}

The novel and important findings of this study are that LDL-C internalization was restored in corrected hepatocyte-like cells and that statin did not induce ER stress in noncorrected hepatocyte-like cells. This data provides proof of concept that these FH-corrected cells are functioning and could be useful in the future as models to compare mutant to corrected cellular response, drug testing or even cell-based therapy work. Overexpression studies have provided evidence that class II LDLR mutants activate the ER stress UPR pathway. Our model served as a tool to determine if a physiologically relevant statininduced LDLR expression would cause ER stress and if correction would alleviate the response. We did not observe ER stress.

It has been hypothesized that class II mutations change the spacing between the highly conserved cysteine residues which interferes with disulfide bond formation and proper LDLR folding $[92,114,122]$. Retention of the class II mutant in the ER as an immature protein suggests it is a transport problem [122] or a protein folding disease [98]. 
We demonstrated previously that modification of the genomic mutation permanently corrects the defect and allows for proper processing to the mature LDLR and receptormedicated endocytosis [189]. We also showed that statin treatment differentially caused accumulation of more total LDLR in the FH class II mutant (predominantly immature) compared to CRISPR corrected (predominantly mature) [189]. This led us to question why the mutant FH cells are retaining more total protein with statin treatment.

Since most of our previous work was performed in cells in the pluripotent state, we initially investigated if receptor-medicated endocytosis was also normalized in differentiated HLC. A time course of internalization of DiI labeled LDL-C showed that even after $24 \mathrm{~h}$, very little DiI could be detected in the mutant HLC, while robust fluorescence is detected in the corrected HLC at both $6 \mathrm{~h}$ and $24 \mathrm{~h}$. When cellular cholesterol is depleted by statin and methyl- $\beta$-cyclodextrin, even at $6 \mathrm{~h}$ neither FH or corrected HLC showed a quantifiable change in cholesterol restoration. Even after $24 \mathrm{~h}$, no statistical change in cellular cholesterol was quantified in FH-HLC. However, in corrected HLC, between $6 \mathrm{~h}$ to $24 \mathrm{~h}$, LDLR activity appears to have been sufficient to restore cellular cholesterol levels likely including within the plasma membrane [312, 319].

Since the class II LDLR mutant is misfolded, we next asked if we could detect a statin mediated accumulation of mutant LDLR in the ER that was resolved in the LDLR corrected cells. We observed NC-HLC LDLR colocalized with calnexin-ER (Figure 22). Previous work with Class II FH563 fibroblasts were also carefully studied through electron microscopy to identify the distribution of the intracellular LDLR [118]. Less than 5\% of LDLR was detectable on the cell surface and in coated pits or vesicles in the endocytic pathway. Instead, most of the class II LDLR was present in membrane extensions of the 
rough ER [118]. It is hypothesized that the extensions at which the mutant LDLR is localized is the site where the ER is blocking exit of the LDLR and appeared to be morphologically similar to the transitional zone of the ER implicated in transport of secretory proteins to the Golgi $[118,127]$. This correlates with our observation in the internalization assays (Figure 21) in which very minimal LDL was internalized by the NCHLC. This small number may represent non-receptor mediated pathways of cholesterol internalization, such as pinocytosis, in addition to the receptor-mediated pathway resulting from the few functional, mature LDL receptors.

One possibility for the accumulation of misfolded LDLR with statin treatment was the statin used. We originally used Lovastatin. Lovastatin was the first FDA approved statin drug and has since been found to also inhibit the proteasome, which is reported to be the mechanism of FH class II LDLR degradation [74, 316, 317]. However, this effect is only seen in the closed-ring $\beta$-lactone-form and does not inhibit HMG-CoA reductase [317]. When we compared the effect on mutant LDLR accumulation of Lovastatin to Rosuvastatin, we detected the same increase in total protein levels of LDLR in NC-cells as well as similar total mature protein between corrected LDLR and wild type control, which demonstrates that Lovastatin proteasome inhibition was not the culprit causing misfolded protein accumulation (Figure 23). The presence of immature LDLR in FH cells has been demonstrated in past studies [122]. A pulse-chase study comparing unaffected and class II FH fibroblasts (the same patient cell type we derived our iPSC from) found that normal fibroblasts' LDLR had been transported to the Golgi for processing to the mature $160-\mathrm{kDa}$ proteins. $95 \%$ of the class II LDLR remained as $120-\mathrm{kDa}$ [122]. The accumulation of mutant LDLR compared to the corrected LDLR was not the result of differential 
transcriptional control, which is what we expected since the cells were treated with statins to inhibit HMG-CoA reductase and upregulate LDLR. We also found that a similar accumulation of mutant LDLR occurs in FH-iPSC and differentiated FH-HLC, indicating mutant LDLR accumulation is a post-translational regulation mechanism. FH class II LDLR proteins are reported to be degraded by two mechanisms, 1) proteasome [119, 320] and 2) lysosome [7, 28, 321]. It is possible that one or both of these systems is overwhelmed by the statin mediated expression induction allowing misfolded protein to accumulate, but then one would expect this to cause ER stress and UPR induction [217, 231].

It has been demonstrated that ER chaperones retain misfolded class II LDLR in the ER. Two-class II mutant LDLRs, C646Y and W556S, were retained in the ER and bound to Grp78 when overexpressed in model Chang cells shown through mass spectrometry and western blot [125]. This was confirmed in CHO cells overexpressing another 2 class II LDLR mutations where there was prolonged binding of Grp78 to mutant LDLR [124]. In addition, ER stress activation in CHO overexpressing mutant G544V LDLR was observed. Spliced XBP1 mRNA was present in the Class II LDLR mutant and absent in overexpressed WT LDLR. Class II mutant LDLR overexpression resulted in a fold induction of phosphorylated-PERK [124]. In contrast, we did not observe an increase in Grp78 expression (Figure 24A, B) in NC-cells treated with Rosuvastatin. We did not detect ER stress activation after Rosuvastatin treatment either (Figure 24C, D, E). Our study specifically used iPSC and derived HLC rather than an overexpression model. It is possible that statins are causing a low-grade chronic ER stress that we were unable to detect. A study of liver samples from untreated patients with chronic hepatitis identified "ERstressed hepatocytes" in clusters scattered in the liver parenchyma [322]. These same 
samples showed protein expression of ATF-6, IRE1, and PERK. However, qPCR of UPRgenes did not show induction of ER stress; instead, genes involved in inflammation and apoptosis were significantly upregulated in these patient samples [322]. Another case study dissected whether liver hepatoxicity was due to statins or another cause [323]. Prolonged latency was seen in patients taking Atorvastatin, Simvastatin, Fluvastatin, and Rosuvastatin, and they presented both hepatocellular and cholestatic patterns of liver injury. Many of these cases resolved after discontinuation of statins. It appeared that statininduced liver injury takes months to years to instigate [323]. It is possible that our cells are expressing ER stress through a non-canonical inflammatory response that we were unable to detect with our methods.

Another chaperone that's been implicated in binding and facilitating the proper folding and maturation of the LDLR is RAP [106]. Mesoderm D (MesD) has also been associated in binding LDL-like receptor proteins [98, 110]. Using this NC and C-HLC model could connect whether or not RAP or MesD are involved in retaining or transporting class II LDLR mutants into or out of the ER. It could also be interesting to investigate if the accumulation of the immature LDLR has an effect on MesD and LDL-like receptor protein interactions and regulation.

It is known that the UPR's purpose is not only to increase folding capacity of the ER, but to also eliminate misfolded proteins by ERAD [324]. The proteasomal degradation pathway also plays a significant role in the degradation of class II LDLR mutants [119]. The $26 \mathrm{~S}$ proteasome is the main protein involved in the ERAD pathway of the cell and degrades misfolded proteins translocated from the ER. Class II LDLR proteins overexpressed in $\mathrm{CHO}$ cells were degraded through the $26 \mathrm{~S}$ proteasome and MG132 
inhibited LDLR degradation [119]. We observed both accumulation of immature LDLR (Figure 23) and no UPR activation (Figure 24). It is possible that degradation is occurring, rather slowly, yet, quickly enough to avoid wholesale UPR. It is also possible the proteasome is not involved in what we are observing. Studies using MG132 proteasome inhibitor can help begin answering these questions.

Our study currently has several limitations. First, differentiation of cells to HLC must be modified to have a monoculture of hepatocytes. Our cells appear to still be a mixture of hepatoblast and hepatocyte, which may answer for the variation in experiments. In addition, our ER stress data must be extended with protein analysis via western blot to assess inflammatory UPR elements like phosphorylated-JNK. Finally, more functional studies for restoration of cholesterol metabolism should be assessed including HMG-CoA reductase activity. To conclude, this data validates that an FH-corrected cell line can physiologically mediate LDLR endocytosis and presents a suitable model to study class II LDLR. In addition, we have determined that the UPR is not activated in a physiological model of class II LDLR HLC. 


\section{CHAPTER V \\ SUMMARY AND CONCLUSIONS}

In this project, we proposed to correct a class II LDLR mutation and follow with further modifying the LDLR and finally create a pre-vascularized liver organoid for implanting these cells. Instead, we were able to create a familial hypercholesterolemia model of non-corrected class II LDLR and corrected LDLR to study cholesterol metabolism and investigate statin-induced immature LDLR accumulation effect on the cell's ER stress system.

We were able to use the CRISPR/Cas9 tool to target a 3-nucleotide deletion in exon 4 of the LDLR of a homozygous patient-derived iPSC. We analyzed LDLR protein levels at the iPSC and HLC level and found a mature LDLR expressed and at lower levels than NC-cells. LDLR-mediated cholesterol internalization was restored in C-HLC as was localization of the LDLR at the plasma membrane. These data provide proof of concept that these FH-corrected cells are functioning and could be useful in the future for cell-based therapy work.

We also are optimizing differentiation of these cells to HLC. There is currently an array of protocols for differentiation of iPSC/ESC to HLC. However, there is no universal standard set because each lab has taken note that what differentiation works for their cells may not work as well in another lab's cells. Efficiency of differentiation can be severely affected by the quality of growth factors and reagents 
used, the type of plastic plate and the amount of cells plated, and the confluence of the cells prior to passaging [290]. Additionally, the growth factors concentration can be variable, as well as species specific. Mouse hepatocyte growth factor is active in mouse cells but not human cells, but human hepatocyte growth factor works well in both human and mouse cells. We are currently in the process of creating a protocol to achieve a high percentage of mature hepatocytes for our studies and definitive endoderm is the primary stage of differentiation. We have observed that both mouse and human Wnt3a are active in our culture for differentiation to definitive endoderm with high expression of Sox 17 and HNF4a. Further studies are necessary to verify these results.

We also investigated the role of ER stress in the non-corrected and corrected FH cell lines. We hypothesized that when treated with statins ER stress would be activated in class II LDLR mutant, but not in mutation corrected FH cells. Overexpression studies have provided evidence that class II LDLR mutants activate the ER stress UPR pathway. Our model served as a tool to determine if a physiologically relevant statin-induced LDLR expression would cause ER stress and if correction would alleviate the response. We could not detect ER stress in the statin treated cells.

Our findings have resulted in many more questions to answer. To begin with, we observed an accumulation of immature LDLR in NC-cells that was not correlative with a greater increase in LDLR transcript levels. Regulation of the cholesterol-LDLR feedback loop is modulated by a set of membrane bound transcription factors called sterol regulatory element binding proteins (SREBPs) [325]. A study using homozygous class II LDLR FH patient fibroblast cells found that the SREBP-2 induced de novo cholesterol synthesis and upregulation of scavenger receptor class B type I (SR-BI) for cholesterol 
uptake [325]. SREBP also plays a role in intracellular lipid accumulation during ER stress [326]. SREBP is usually retained in the ER by Insig. However, during ER stress, Insig translation rapidly declines allowing SREBP to escape the ER and activate transcription of genes for lipid synthesis and uptake. We did not check the expression of SREBP or its associated genes. In addition, changes could be occurring at the epigenetic level. It would be interesting to evaluate through microarray which genes are up or downregulated in an FH and corrected HLC cell line. This could be followed with a CHIP assay to determine if SREBPs interaction with LDLR promoter is modified.

Statins are the most prescribed medication worldwide. Many studies have evaluated the risks associated with short-and-long term use of the drug. Generally, statins are well-tolerated. However, up to $15 \%$ of patients develop adverse effects to statins, including myalgia. Even scarcer are reports of stain induced liver injury. A case study dissected whether liver hepatoxicity was due to statins or another cause [323]. Prolonged latency was seen in patients taking Atorvastatin, Simvastatin, Fluvastatin, and Rosuvastatin, and they presented both hepatocellular and cholestatic patterns of liver injury. Many of these cases resolved after discontinuation of statins. One patient who died also had pre-existing alcoholic cirrhosis. Another patient who had restarted on statins rapidly redeveloped acute injury. It also appears that statin-induced liver injury takes months to years to instigate [323]. HoFH patients can begin treatment on statins as young as 6 years old. Can a patient with a liver disease like FH be more susceptible to statin-induced liver injury and not know it until it has become severe?

Statins are classified category $\mathrm{X}$, meaning the drug should not be taken during pregnancy $[327,328]$ because of studies that have shown problems in the fetus of animals 
or pregnant women who mistakenly took the medication without being aware of their pregnancy. Human iPSC/ESC are useful tools in studying early human development and could be a useful source to determine what, if any, effects occur with statin exposure in NC versus C-iPSC. A study treating iPSC long and short-term with statins before forming them into embryoid bodies could reveal an array of information on which genes are changed because of treatment.

To conclude, we have established a cell line model to study class II LDLR mutations that could provide a platform to answer questions. 


\section{REFERENCES}

1. de Ferranti, S.D., et al., Prevalence of Familial Hypercholesterolemia in the 1999 to 2012 United States National Health and Nutrition Examination Surveys (NHANES). Circulation, 2016. 133(11): p. 1067-72.

2. Sjouke, B., et al., Homozygous autosomal dominant hypercholesterolaemia: prevalence, diagnosis, and current and future treatment perspectives. Curr Opin Lipidol, 2015. 26(3): p. 200-9.

3. Huff, M.W., J.M. Assini, and R.A. Hegele, Gene therapy for hypercholesterolemia: sweet dreams and flying machines. Circ Res, 2014. 115(6): p. $542-5$.

4. Brown, M.S. and J.L. Goldstein, A receptor-mediated pathway for cholesterol homeostasis. Science, 1986. 232(4746): p. 34-47.

5. Shu-Yu, C., et al., Lipoprotein in cholesterol transport: Highlights and recent insights into its structural basis and functional mechanism. Chinese Physics B, 2018. 27(2): p. 028702.

6. Burns, M.P. and G.W. Rebeck, Intracellular cholesterol homeostasis and amyloid precursor protein processing. Biochim Biophys Acta, 2010. 1801(8): p. 853-9.

7. Brown, M.S. and J.L. Goldstein, Receptor-mediated control of cholesterol metabolism. Science, 1976. 191(4223): p. 150-4. 
8. Endo, A., M. Kuroda, and K. Tanzawa, Competitive inhibition of 3-hydroxy-3methylglutaryl coenzyme A reductase by ML-236A and ML-236B fungal metabolites, having hypocholesterolemic activity. FEBS Lett, 1976. 72(2): p. 3236.

9. Gelissen, I.C. and A.J. McLachlan, The pharmacogenomics of statins. Pharmacol Res, 2014. 88: p. 99-106.

10. Feingold, K.R., et al., De novo sterologenesis in intact primates. J Lab Clin Med, 1982. 100(3): p. 405-10.

11. Brown, M.S., S.E. Dana, and J.L. Goldstein, Regulation of 3-hydroxy-3methylglutaryl coenzyme A reductase activity in human fibroblasts by lipoproteins. Proc Natl Acad Sci U S A, 1973. 70(7): p. 2162-6.

12. Goldstein, J.L., et al., Steroid requirements for suppression of HMG CoA reductase activity in cultured human fibroblasts. Adv Exp Med Biol, 1975. 63: p. 77-84.

13. Brown, M.S., J.R. Faust, and J.L. Goldstein, Role of the low density lipoprotein receptor in regulating the content of free and esterified cholesterol in human fibroblasts. J Clin Invest, 1975. 55(4): p. 783-93.

14. Singh, S. and V. Bittner, Familial Hypercholesterolemia-Epidemiology, Diagnosis, and Screening. Current Atherosclerosis Reports, 2015. 17(2): p. 3.

15. Thompson, G.R., Managing homozygous familial hypercholesterolaemia from cradle to grave. Atheroscler Suppl, 2015. 18: p. 16-20. 
16. Sanna, C., et al., Homozygous familial hypercholesterolemia in childhood: Genotype-phenotype description, established therapies and perspectives. Atherosclerosis, 2016. 247: p. 97-104.

17. Gidding, S.S., The complexities of homozygous familial hypercholesterolemia management. Pediatr Transplant, 2016. 20(8): p. 1020-1021.

18. Widhalm, K., et al., Sudden death in a 4-year-old boy: a near-complete occlusion of the coronary artery caused by an aggressive low-density lipoprotein receptor mutation (W556R) in homozygous familial hypercholesterolemia. J Pediatr, 2011. 158(1): p. 167.

19. Wang, A., et al., Systematic Review of Low-Density Lipoprotein Cholesterol Apheresis for the Treatment of Familial Hypercholesterolemia. J Am Heart Assoc, 2016. 5(7).

20. Safarova, M.S. and I.J. Kullo, My Approach to the Patient With Familial Hypercholesterolemia. Mayo Clin Proc, 2016. 91(6): p. 770-86.

21. Hemphill, L.C., Familial hypercholesterolemia: current treatment options and patient selection for low-density lipoprotein apheresis. J Clin Lipidol, 2010. 4(5): p. 346-9.

22. Raal, F.J., et al., PCSK9 inhibition with evolocumab (AMG 145) in heterozygous familial hypercholesterolaemia (RUTHERFORD-2): a randomised, double-blind, placebo-controlled trial. Lancet, 2015. 385(9965): p. 331-40.

23. Raal, F.J., et al., Inhibition of PCSK9 with evolocumab in homozygous familial hypercholesterolaemia (TESLA Part B): a randomised, double-blind, placebocontrolled trial. Lancet, 2015. 385(9965): p. 341-50. 
24. Gouni-Berthold, I. and H.K. Berthold, Mipomersen and lomitapide: Two new drugs for the treatment of homozygous familial hypercholesterolemia. Atheroscler Suppl, 2015. 18: p. 28-34.

25. Endo, A., A gift from nature: the birth of the statins. Nat Med, 2008. 14(10): p. $1050-2$.

26. Gidding, S.S., et al., The Agenda for Familial Hypercholesterolemia: A Scientific Statement From the American Heart Association. Circulation, 2015. 132(22): p. 2167-92.

27. Wu, N.Q. and J.J. Li, PCSK9 gene mutations and low-density lipoprotein cholesterol. Clin Chim Acta, 2014. 431: p. 148-53.

28. Zhang, D.W., et al., Binding of proprotein convertase subtilisin/kexin type 9 to epidermal growth factor-like repeat A of low density lipoprotein receptor decreases receptor recycling and increases degradation. J Biol Chem, 2007. 282(25): p. 18602-12.

29. Gu, H.M., et al., Characterization of the role of EGF-A of low density lipoprotein receptor in PCSK9 binding. J Lipid Res, 2013. 54(12): p. 3345-57.

30. Chaparro-Riggers, J., et al., Increasing serum half-life and extending cholesterol lowering in vivo by engineering antibody with $\mathrm{pH}$-sensitive binding to PCSK9. J Biol Chem, 2012. 287(14): p. 11090-7.

31. Dubuc, G., et al., Statins upregulate PCSK9, the gene encoding the proprotein convertase neural apoptosis-regulated convertase-1 implicated in familial hypercholesterolemia. Arterioscler Thromb Vasc Biol, 2004. 24(8): p. 1454-9. 
32. Mombelli, G. and C. Pavanello, Novel therapeutic strategies for the homozygous familial hypercholesterolemia. Recent Pat Cardiovasc Drug Discov, 2013. 8(2): p. $143-50$.

33. Mansoorian, M., et al., Liver transplantation as a definitive treatment for familial hypercholesterolemia: A series of 36 cases. Pediatr Transplant, 2015. 19(6): p. $605-11$.

34. Alim, A., et al., Liver transplantation for homozygote familial hypercholesterolemia: the only curative treatment. Pediatr Transplant, 2016. 20(8): p. 1060-1064.

35. Page, M.M., et al., Liver transplantation for the treatment of homozygous familial hypercholesterolaemia in an era of emerging lipid-lowering therapies. Intern Med J, 2014. 44(6): p. 601-4.

36. Maiorana, A., et al., Preemptive liver transplantation in a child with familial hypercholesterolemia. Pediatr Transplant, 2011. 15(2): p. E25-9.

37. Greco, M., et al., Progressive Aortic Stenosis in Homozygous Familial Hypercholesterolemia After Liver Transplant. Pediatrics, 2016. 138(5).

38. Versland, M.R., C.H. Wu, and G.Y. Wu, Strategies for gene therapy in the liver. Semin Liver Dis, 1992. 12(3): p. 332-9.

39. Raper, S.E., J.M. Wilson, and M. Grossman, Retroviral-mediated gene transfer in human hepatocytes. Surgery, 1992. 112(2): p. 333-9; discussion 339-40.

40. Grossman, M., S.E. Raper, and J.M. Wilson, Towards liver-directed gene therapy: retrovirus-mediated gene transfer into human hepatocytes. Somat Cell Mol Genet, 1991. 17(6): p. 601-7. 
41. Havel, R.J., N. Yamada, and D.M. Shames, Watanabe heritable hyperlipidemic rabbit. Animal model for familial hypercholesterolemia. Arteriosclerosis, 1989. 9(1 Suppl): p. I33-8.

42. Gunsalus, J.R., et al., Reduction of serum cholesterol in Watanabe rabbits by xenogeneic hepatocellular transplantation. Nat Med, 1997. 3(1): p. 48-53.

43. Wiederkehr, J.C., G.T. Kondos, and R. Pollak, Hepatocyte transplantation for the low-density lipoprotein receptor-deficient state. A study in the Watanabe rabbit. Transplantation, 1990. 50(3): p. 466-71.

44. Wilson, J.M., et al., Correction of the genetic defect in hepatocytes from the Watanabe heritable hyperlipidemic rabbit. Proc Natl Acad Sci U S A, 1988. 85(12): p. 4421-5.

45. Chowdhury, J.R., et al., Long-term improvement of hypercholesterolemia after ex vivo gene therapy in LDLR-deficient rabbits. Science, 1991. 254(5039): p. 18025.

46. Suda, T., et al., Progress toward liver-based gene therapy. Hepatology Research, 2009. 39(4): p. 325-340.

47. Herz, J. and R.D. Gerard, Adenovirus-mediated transfer of low density lipoprotein receptor gene acutely accelerates cholesterol clearance in normal mice. Proc Natl Acad Sci U S A, 1993. 90(7): p. 2812-6.

48. Kozarsky, K., M. Grossman, and J.M. Wilson, Adenovirus-mediated correction of the genetic defect in hepatocytes from patients with familial hypercholesterolemia. Somat Cell Mol Genet, 1993. 19(5): p. 449-58. 
49. Makdisi, W.J., C.H. Wu, and G.Y. Wu, Methods of gene transfer into hepatocytes: progress toward gene therapy. Prog Liver Dis, 1992. 10: p. 1-24.

50. Grossman, M., et al., Successful ex vivo gene therapy directed to liver in a patient with familial hypercholesterolaemia. Nat Genet, 1994. 6(4): p. 335-41.

51. Grossman, M., et al., A pilot study of ex vivo gene therapy for homozygous familial hypercholesterolaemia. Nat Med, 1995. 1(11): p. 1148-54.

52. Raper, S.E. and J.M. Wilson, Cell transplantation in liver-directed gene therapy. Cell Transplant, 1993. 2(5): p. 381-400; discussion 407-10.

53. Gupta, S., et al., Hepatocyte transplantation: progress toward liver repopulation. Prog Liver Dis, 1996. 14: p. 199-222.

54. Grossman, M. and J.M. Wilson, Retroviruses: delivery vehicle to the liver. Curr Opin Genet Dev, 1993. 3(1): p. 110-4.

55. Gupta, S., et al., Position-specific gene expression in the liver lobule is directed by the microenvironment and not by the previous cell differentiation state. $\mathrm{J}$ Biol Chem, 1999. 274(4): p. 2157-65.

56. Gupta, S., et al., Hepatocytes exhibit superior transgene expression after transplantation into liver and spleen compared with peritoneal cavity or dorsal fat pad: implications for hepatic gene therapy. Hum Gene Ther, 1994. 5(8): p. 959-67.

57. Guha, C., et al., Amelioration of radiation-induced liver damage in partially hepatectomized rats by hepatocyte transplantation. Cancer Res, 1999. 59(23): p. 5871-4. 
58. Gupta, S., G.R. Gorla, and A.N. Irani, Hepatocyte transplantation: emerging insights into mechanisms of liver repopulation and their relevance to potential therapies. J Hepatol, 1999. 30(1): p. 162-70.

59. Gupta, S., D.R. LaBrecque, and D.A. Shafritz, Mitogenic effects of hepatic stimulator substance on cultured nonparenchymal liver epithelial cells. Hepatology, 1992. 15(3): p. 485-91.

60. Gupta, S., et al., Transplanted hepatocytes proliferate differently after CCl4 treatment and hepatocyte growth factor infusion. Am J Physiol, 1999. 276(3 Pt 1): p. G629-38.

61. Xu, F., et al., Dual roles for cholesterol in mammalian cells. Proc Natl Acad Sci U S A, 2005. 102(41): p. 14551-6.

62. Goldstein, J.L. and M.S. Brown, Lipoprotein receptors, cholesterol metabolism, and atherosclerosis. Arch Pathol, 1975. 99(4): p. 181-4.

63. Kirby, T.J., Cataracts produced by triparanol. (MER-29). Trans Am Ophthalmol Soc, 1967. 65: p. 494-543.

64. Avigan, J., et al., Mechanism of action of MER-29, an inhibitor of cholesterol biosynthesis. Biochem Biophys Res Commun, 1960. 2: p. 63-5.

65. Avigan, J. and D. Steinberg, Deposition of desmosterol in the lesions of experimental atherosclerosis. Lancet, 1962. 1(7229): p. 572.

66. Steinberg, D., J. Avigan, and E.B. Feigelson, Identification of 24dehydrocholesterol in the serum of patients treated with MER-29. Prog Cardiovasc Dis, 1960. 2: p. 586-92. 
67. Endo, A., A historical perspective on the discovery of statins. Proc Jpn Acad Ser B Phys Biol Sci, 2010. 86(5): p. 484-93.

68. Knauss, H.J., J.W. Porter, and G. Wasson, The biosynthesis of mevalonic acid from 1-Cl4-acetate by a rat liver enzyme system. J Biol Chem, 1959. 234: $\mathrm{p}$. 2835-40.

69. Bucher Nl Fau - Swaffield, M.N. and M.N. Swaffield, Rate of incorporation of [6-14C]orotic acid into uridine 5'-triphosphate and cytidine 5'-triphosphate and nuclear ribonucleic acid in regenerating rat liver. (0006-3002 (Print)).

70. Endo, A. and M. Kuroda, Citrinin, an inhibitor of cholesterol synthesis. J Antibiot (Tokyo), 1976. 29(8): p. 841-3.

71. Tanzawa, K., M. Kuroda, and A. Endo, Time-dependent, irreversible inhibition of 3-hydroxy-3-methylglutaryl-coenzyme A reductase by the antibiotic citrinin. Biochim Biophys Acta, 1977. 488(1): p. 97-101.

72. Endo, A., M. Kuroda, and Y. Tsujita, $M L-236 A, M L-236 B$, and $M L-236 C$, new inhibitors of cholesterogenesis produced by Penicillium citrinium. J Antibiot (Tokyo), 1976. 29(12): p. 1346-8.

73. Endo, A., et al., Effects of ML-236B on cholesterol metabolism in mice and rats: lack of hypocholesterolemic activity in normal animals. Biochim Biophys Acta, 1979. 575(2): p. 266-76.

74. Yamamoto, A., H. Sudo, and A. Endo, Therapeutic effects of ML-236B in primary hypercholesterolemia. Atherosclerosis, 1980. 35(3): p. 259-66. 
75. Alberts, A.W., et al., Mevinolin: a highly potent competitive inhibitor of hydroxymethylglutaryl-coenzyme A reductase and a cholesterol-lowering agent. Proc Natl Acad Sci U S A, 1980. 77(7): p. 3957-61.

76. Steinberg, D., Thematic review series: the pathogenesis of atherosclerosis. An interpretive history of the cholesterol controversy, part V: the discovery of the statins and the end of the controversy. J Lipid Res, 2006. 47(7): p. 1339-51.

77. Endo, A., Monacolin K, a new hypocholesterolemic agent produced by a Monascus species. J Antibiot (Tokyo), 1979. 32(8): p. 852-4.

78. Vagelos, P.R., Are prescription drug prices high? Science, 1991. 252(5009): p. $1080-4$.

79. DeGorter, M.K., et al., Clinical and pharmacogenetic predictors of circulating atorvastatin and rosuvastatin concentrations in routine clinical care. Circ Cardiovasc Genet, 2013. 6(4): p. 400-8.

80. Mihos, C.G., A.M. Pineda, and O. Santana, Cardiovascular effects of statins, beyond lipid-lowering properties. Pharmacol Res, 2014. 88: p. 12-9.

81. Bifulco, M. and A. Endo, Statin: new life for an old drug. Pharmacol Res, 2014. 88: p. $1-2$.

82. Van Wyhe, R.D., O.M. Rahal, and W.A. Woodward, Effect of statins on breast cancer recurrence and mortality: a review. Breast Cancer (Dove Med Press), 2017. 9: p. 559-565.

83. Pisanti, S., et al., Novel prospects of statins as therapeutic agents in cancer. Pharmacol Res, 2014. 88: p. 84-98. 
84. Ferri, N. and A. Corsini, Clinical evidence of statin therapy in non-dyslipidemic disorders. Pharmacol Res, 2014. 88: p. 20-30.

85. Dobesh, P.P. and K.M. Olsen, Statins role in the prevention and treatment of sepsis. Pharmacol Res, 2014. 88: p. 31-40.

86. Lippi, G., E.J. Favaloro, and F. Sanchis-Gomar, Venous thrombosis associated with HMG-CoA reductase inhibitors. Semin Thromb Hemost, 2013. 39(5): p. 515-32.

87. Jeon, H. and S.C. Blacklow, Structure and physiologic function of the low-density lipoprotein receptor. Annu Rev Biochem, 2005. 74: p. 535-62.

88. Lindgren, V., et al., Human genes involved in cholesterol metabolism: chromosomal mapping of the loci for the low density lipoprotein receptor and 3hydroxy-3-methylglutaryl-coenzyme A reductase with cDNA probes. Proc Natl Acad Sci U S A, 1985. 82(24): p. 8567-71.

89. Yamamoto, T., et al., The human LDL receptor: a cysteine-rich protein with multiple Alu sequences in its mRNA. Cell, 1984. 39(1): p. 27-38.

90. Al-Allaf, F.A., et al., LDLR-Gene therapy for familial hypercholesterolaemia: problems, progress, and perspectives. Int Arch Med, 2010. 3: p. 36.

91. Hobbs, H.H., M.S. Brown, and J.L. Goldstein, Molecular genetics of the LDL receptor gene in familial hypercholesterolemia. Hum Mutat, 1992. 1(6): p. 44566.

92. Hobbs, H.H., et al., The LDL receptor locus in familial hypercholesterolemia: mutational analysis of a membrane protein. Annu Rev Genet, 1990. 24: p. 13370. 
93. Russell, D.W., et al., cDNA cloning of the bovine low density lipoprotein receptor: feedback regulation of a receptor mRNA. Proc Natl Acad Sci U S A, 1983. 80(24): p. 7501-5.

94. Smith, J.R., et al., Identification of nucleotides responsible for enhancer activity of sterol regulatory element in low density lipoprotein receptor gene. (0021-9258 (Print)).

95. Rabès, M.V.a.J.-P., Missense Mutation in the LDLR Gene: A Wide Spectrum in the Severity of Familial Hypercholesterolemia, in Mutations in Human Genetic Disease, D.N.C.a.J.-M. Chen, Editor. 2012, IntechOpen.

96. Kwon, H.J., et al., Molecular basis for LDL receptor recognition by PCSK9. Proc Natl Acad Sci U S A, 2008. 105(6): p. 1820-5.

97. Davis, C.G., et al., Deletion of clustered O-linked carbohydrates does not impair function of low density lipoprotein receptor in transfected fibroblasts. J Biol Chem, 1986. 261(6): p. 2828-38.

98. Gent, J. and I. Braakman, Low-density lipoprotein receptor structure and folding. Cell Mol Life Sci, 2004. 61(19-20): p. 2461-70.

99. Radford, S.E., Co-translocational misfolding in the ER of living cells. Nat Struct Biol, 2003. 10(3): p. 153-4.

100. Jansens, A., E. van Duijn, and I. Braakman, Coordinated Nonvectorial Folding in a Newly Synthesized Multidomain Protein. Science, 2002. 298(5602): p. 2401.

101. Saha, S., et al., Solution structure of the LDL receptor EGF-AB pair: a paradigm for the assembly of tandem calcium binding EGF domains. Structure, 2001. 9(6): p. 451-6. 
102. Kurniawan, N.D., et al., NMR structure and backbone dynamics of a concatemer of epidermal growth factor homology modules of the human low-density lipoprotein receptor. J Mol Biol, 2001. 311(2): p. 341-56.

103. Lee, A.S., The ER chaperone and signaling regulator GRP78/BiP as a monitor of endoplasmic reticulum stress. Methods, 2005. 35(4): p. 373-81.

104. Little, E., et al., The glucose-regulated proteins (GRP78 and GRP94): functions, gene regulation, and applications. Crit Rev Eukaryot Gene Expr, 1994. 4(1): p. 118.

105. Ellgaard, L. and A. Helenius, Quality control in the endoplasmic reticulum. Nature Reviews Molecular Cell Biology, 2003. 4(3): p. 181091.

106. Bu, G. and A.L. Schwartz, RAP, a novel type of ER chaperone. Trends Cell Biol, 1998. 8(7): p. 272-6.

107. Willnow, T.E., et al., RAP, a specialized chaperone, prevents ligand-induced ER retention and degradation of $L D L$ receptor-related endocytic receptors. Embo $\mathrm{j}$, 1996. 15(11): p. 2632-9.

108. Willnow, T.E., et al., Functional expression of low density lipoprotein receptorrelated protein is controlled by receptor-associated protein in vivo. Proc Natl Acad Sci U S A, 1995. 92(10): p. 4537-41.

109. Willnow, T.E., Receptor-associated protein (RAP): a specialized chaperone for endocytic receptors. Biol Chem, 1998. 379(8-9): p. 1025-31.

110. Hsieh, J.-C., et al., Mesd Encodes an LRP5/6 Chaperone Essential for Specification of Mouse Embryonic Polarity. Cell, 2003. 112(3): p. 355-67. 
111. Culi, J. and R.S. Mann, Boca, an endoplasmic reticulum protein required for wingless signaling and trafficking of LDL receptor family members in Drosophila. (0092-8674 (Print)).

112. Culi, J., T.A. Springer, and R.S. Mann, Boca-dependent maturation of $\beta$ propeller/EGF modules in low-density lipoprotein receptor proteins. The EMBO Journal, 2004. 23(6): p. 1372-1380.

113. Wijers, M., J.A. Kuivenhoven, and B. van de Sluis, The life cycle of the lowdensity lipoprotein receptor: insights from cellular and in-vivo studies. Curr Opin Lipidol, 2015. 26(2): p. 82-7.

114. Russell, D.W., V. Esser, and H.H. Hobbs, Molecular basis of familial hypercholesterolemia. Arteriosclerosis, 1989. 9(1 Suppl): p. I8-13.

115. Koivisto, U.M., A.L. Hubbard, and I. Mellman, A novel cellular phenotype for familial hypercholesterolemia due to a defect in polarized targeting of LDL receptor. Cell, 2001. 105(5): p. 575-85.

116. Tolleshaug, H., et al., The LDL receptor locus in familial hypercholesterolemia: multiple mutations disrupt transport and processing of a membrane receptor. Cell, 1983. 32(3): p. 941-51.

117. Esser, V. and D.W. Russell, Transport-deficient mutations in the low density lipoprotein receptor. Alterations in the cysteine-rich and cysteine-poor regions of the protein block intracellular transport. J Biol Chem, 1988. 263(26): p. 1327681. 
118. Pathak, R.K., et al., Immunocytochemical localization of mutant low density lipoprotein receptors that fail to reach the Golgi complex. Journal of Cell Biology, 1988. 106(6): p. 1831-1841.

119. Li, Y., et al., Degradation of the LDL receptor class 2 mutants is mediated by a proteasome-dependent pathway. J Lipid Res, 2004. 45(6): p. 1084-91.

120. Beissinger, M. and J. Buchner, How chaperones fold proteins. Biol Chem, 1998. 379(3): p. 245-59.

121. Carrell, R.W. and D.A. Lomas, Conformational Disease. The Lancet, 1997. 350(9071): p. 134-138.

122. Yamamoto, T., et al., Deletion in cysteine-rich region of $L D L$ receptor impedes transport to cell surface in WHHL rabbit. Science, 1986. 232(4755): p. 1230-7.

123. Ranheim, T., et al., Model system for phenotypic characterization of sequence variations in the LDL receptor gene. Clin Chem, 2006. 52(8): p. 1469-79.

124. Sorensen, S., et al., Retention of mutant low density lipoprotein receptor in endoplasmic reticulum (ER) leads to ER stress. J Biol Chem, 2006. 281(1): p. 468-76.

125. Jorgensen, M.M., et al., Grp78 is involved in retention of mutant low density lipoprotein receptor protein in the endoplasmic reticulum. J Biol Chem, 2000. 275(43): p. 33861-8.

126. Esser, V., et al., Mutational analysis of the ligand binding domain of the low density lipoprotein receptor. J Biol Chem, 1988. 263(26): p. 13282-90.

127. Palade, G., Intracellular aspects of the process of protein synthesis. Science, 1975. 189(4200): p. 347-58. 
128. Genetic Mapping. 2018 October 21, 2015 [cited 2018 January 8]; Available from: https://www.genome.gov/10000715/genetic-mapping-fact-sheet/.

129. Alim, A., et al., Liver transplantation for homozygote familial hypercholesterolemia: the only curative treatment. Pediatric Transplantation, 2016. 20(8): p. 1060-1064.

130. OMIM Gene Map Statistics. Online Mendelian Inheritance in Man 2018 February 20, 2018; Available from: http://www.omim.org/statistics/geneMap.

131. Gupta, R.M. and K. Musunuru, Expanding the genetic editing tool kit: ZFNs, TALENs, and CRISPR-Cas9. J Clin Invest, 2014. 124(10): p. 4154-61.

132. Li, X. and W.D. Heyer, Homologous recombination in DNA repair and DNA damage tolerance. Cell Res, 2008. 18(1): p. 99-113.

133. Capecchi, M.R., Altering the genome by homologous recombination. Science, 1989. 244(4910): p. 1288-92.

134. Mak, T.W., Gene targeting in embryonic stem cells scores a knockout in Stockholm. Cell, 2007. 131(6): p. 1027-31.

135. Kucherlapati, R.S., et al., Homologous recombination between plasmids in mammalian cells can be enhanced by treatment of input DNA. Proc Natl Acad Sci U S A, 1984. 81(10): p. 3153-7.

136. Wong, E.A. and M.R. Capecchi, Analysis of homologous recombination in cultured mammalian cells in transient expression and stable transformation assays. Somat Cell Mol Genet, 1986. 12(1): p. 63-72. 
137. Brenner, D.A., A.C. Smigocki, and R.D. Camerini-Otero, Effect of insertions, deletions, and double-strand breaks on homologous recombination in mouse L cells. Mol Cell Biol, 1985. 5(4): p. 684-91.

138. Lin, F.L., K. Sperle, and N. Sternberg, Model for homologous recombination during transfer of DNA into mouse L cells: role for DNA ends in the recombination process. Mol Cell Biol, 1984. 4(6): p. 1020-34.

139. Brenner, D.A., A.C. Smigocki, and R.D. Camerini-Otero, Double-strand gap repair results in homologous recombination in mouse L cells. Proc Natl Acad Sci U S A, 1986. 83(6): p. 1762-6.

140. Smith, A.J. and P. Berg, Homologous recombination between defective neo genes in mouse 3 T6 cells. Cold Spring Harb Symp Quant Biol, 1984. 49: p. 171-81.

141. Doetschman, T., et al., Targetted correction of a mutant HPRT gene in mouse embryonic stem cells. Nature, 1987. 330(6148): p. 576-8.

142. Kuehn, M.R., et al., A potential animal model for Lesch-Nyhan syndrome through introduction of HPRT mutations into mice. Nature, 1987. 326(6110): p. 295-8.

143. Hooper, M., et al., HPRT-deficient (Lesch-Nyhan) mouse embryos derived from germline colonization by cultured cells. Nature, 1987. 326(6110): p. 292-5.

144. Hasty, P., et al., Target frequency and integration pattern for insertion and replacement vectors in embryonic stem cells. Mol Cell Biol, 1991. 11(9): p. 450917.

145. Hotta, A. and S. Yamanaka, From Genomics to Gene Therapy: Induced Pluripotent Stem Cells Meet Genome Editing. Annu Rev Genet, 2015. 49: p. 4770. 
146. Jeggo, P.A., DNA breakage and repair. Adv Genet, 1998. 38: p. 185-218.

147. Ganesh, A., P. North, and J. Thacker, Repair and misrepair of site-specific DNA double-strand breaks by human cell extracts. Mutat Res, 1993. 299(3-4): p. 2519.

148. Fishman-Lobell, J., N. Rudin, and J.E. Haber, Two alternative pathways of double-strand break repair that are kinetically separable and independently modulated. Mol Cell Biol, 1992. 12(3): p. 1292-303.

149. Fishman-Lobell, J. and J.E. Haber, Removal of nonhomologous DNA ends in double-strand break recombination: the role of the yeast ultraviolet repair gene RADl. Science, 1992. 258(5081): p. 480-4.

150. North, P., A. Ganesh, and J. Thacker, The rejoining of double-strand breaks in DNA by human cell extracts. Nucleic Acids Res, 1990. 18(21): p. 6205-10.

151. Rouet, P., F. Smih, and M. Jasin, Introduction of double-strand breaks into the genome of mouse cells by expression of a rare-cutting endonuclease. Mol Cell Biol, 1994. 14(12): p. 8096-106.

152. Choulika, A., et al., Induction of homologous recombination in mammalian chromosomes by using the I-SceI system of Saccharomyces cerevisiae. Mol Cell Biol, 1995. 15(4): p. 1968-73.

153. Cohen-Tannoudji, M., et al., I-SceI-induced gene replacement at a natural locus in embryonic stem cells. Mol Cell Biol, 1998. 18(3): p. 1444-8.

154. Porteus, M.H. and D. Carroll, Gene targeting using zinc finger nucleases. Nat Biotechnol, 2005. 23(8): p. 967-73. 
155. Chandrasegaran, S. and J. Smith, Chimeric restriction enzymes: what is next? Biol Chem, 1999. 380(7-8): p. 841-8.

156. Kim, Y.G., J. Cha, and S. Chandrasegaran, Hybrid restriction enzymes: zinc finger fusions to Fok I cleavage domain. Proc Natl Acad Sci U S A, 1996. 93(3): p. $1156-60$.

157. Porteus, M.H. and D. Baltimore, Chimeric nucleases stimulate gene targeting in human cells. Science, 2003. 300(5620): p. 763.

158. Urnov, F.D., et al., Highly efficient endogenous human gene correction using designed zinc-finger nucleases. Nature, 2005. 435(7042): p. 646-51.

159. Wolfe, S.A., L. Nekludova, and C.O. Pabo, DNA recognition by Cys2His2 zinc finger proteins. Annu Rev Biophys Biomol Struct, 2000. 29: p. 183-212.

160. Moscou, M.J. and A.J. Bogdanove, A simple cipher governs DNA recognition by TAL effectors. (1095-9203 (Electronic)).

161. Boch, J., et al., Breaking the Code of DNA Binding Specificity of TAL-Type III Effectors. Science, 2009. 326(5959): p. 1509.

162. Reyon, D., et al., FLASH assembly of TALENs for high-throughput genome editing. Nat Biotechnol, 2012. 30(5): p. 460-5.

163. Guilinger, J.P., et al., Broad specificity profiling of TALENs results in engineered nucleases with improved DNA-cleavage specificity. Nat Methods, 2014. 11(4): p. 429-35.

164. Wang, H., M. La Russa, and L.S. Qi, CRISPR/Cas9 in Genome Editing and Beyond. Annu Rev Biochem, 2016. 85: p. 227-64. 
165. Yin, H., K.J. Kauffman, and D.G. Anderson, Delivery technologies for genome editing. Nat Rev Drug Discov, 2017. 16(6): p. 387-399.

166. Ran, F.A., et al., Genome engineering using the CRISPR-Cas9 system. Nat Protoc, 2013. 8(11): p. 2281-2308.

167. Lander, Eric S., The Heroes of CRISPR. Cell, 2016. 164(1): p. 18-28.

168. Ishino, Y., et al., Nucleotide sequence of the iap gene, responsible for alkaline phosphatase isozyme conversion in Escherichia coli, and identification of the gene product. J Bacteriol, 1987. 169(12): p. 5429-33.

169. Mojica, F.J., et al., Biological significance of a family of regularly spaced repeats in the genomes of Archaea, Bacteria and mitochondria. Mol Microbiol, 2000. 36(1): p. 244-6.

170. Mojica, F.J., et al., Intervening sequences of regularly spaced prokaryotic repeats derive from foreign genetic elements. J Mol Evol, 2005. 60(2): p. 174-82.

171. Pourcel, C., G. Salvignol, and G. Vergnaud, CRISPR elements in Yersinia pestis acquire new repeats by preferential uptake of bacteriophage DNA, and provide additional tools for evolutionary studies. Microbiology, 2005. 151(Pt 3): p. 65363.

172. Li, H.L., et al., Efficient genomic correction methods in human iPS cells using CRISPR-Cas9 system. Methods, 2016. 101: p. 27-35.

173. Barrangou, R., et al., CRISPR provides acquired resistance against viruses in prokaryotes. Science, 2007. 315(5819): p. 1709-12. 
174. Bolotin, A., et al., Clustered regularly interspaced short palindrome repeats (CRISPRs) have spacers of extrachromosomal origin. Microbiology, 2005. 151(Pt 8): p. 2551-61.

175. Makarova, K.S., et al., A putative RNA-interference-based immune system in prokaryotes: computational analysis of the predicted enzymatic machinery, functional analogies with eukaryotic RNAi, and hypothetical mechanisms of action. Biol Direct, 2006. 1: p. 7.

176. Brouns, S.J., et al., Small CRISPR RNAs guide antiviral defense in prokaryotes. Science, 2008. 321(5891): p. 960-4.

177. Marraffini, L.A. and E.J. Sontheimer, CRISPR interference limits horizontal gene transfer in staphylococci by targeting DNA. Science, 2008. 322(5909): p. 1843-5.

178. Hale, C.R., et al., RNA-guided RNA cleavage by a CRISPR RNA-Cas protein complex. Cell, 2009. 139(5): p. 945-56.

179. Garneau, J.E., et al., The CRISPR/Cas bacterial immune system cleaves bacteriophage and plasmid DNA. Nature, 2010. 468(7320): p. 67-71.

180. Deveau, H., et al., Phage response to CRISPR-encoded resistance in Streptococcus thermophilus. J Bacteriol, 2008. 190(4): p. 1390-400.

181. Horvath, P., et al., Diversity, activity, and evolution of CRISPR loci in Streptococcus thermophilus. J Bacteriol, 2008. 190(4): p. 1401-12.

182. Deltcheva, E., et al., CRISPR RNA maturation by trans-encoded small RNA and host factor RNase III. Nature, 2011. 471(7340): p. 602-7. 
183. Sapranauskas, R., et al., The Streptococcus thermophilus CRISPR/Cas system provides immunity in Escherichia coli. Nucleic Acids Res, 2011. 39(21): p. 927582.

184. Gasiunas, G., et al., Cas9-crRNA ribonucleoprotein complex mediates specific DNA cleavage for adaptive immunity in bacteria. Proc Natl Acad Sci U S A, 2012. 109(39): p. E2579-86.

185. Jinek, M., et al., A programmable dual-RNA-guided DNA endonuclease in adaptive bacterial immunity. Science, 2012. 337(6096): p. 816-21.

186. Cong, L., et al., Multiplex genome engineering using CRISPR/Cas systems. Science, 2013. 339(6121): p. 819-23.

187. Jarrett, K.E., et al., Somatic genome editing with CRISPR/Cas 9 generates and corrects a metabolic disease. Sci Rep, 2017. 7: p. 44624.

188. Liu, Y., et al., Lack of MTTP Activity in Pluripotent Stem Cell-Derived Hepatocytes and Cardiomyocytes Abolishes apoB Secretion and Increases Cell Stress. Cell Rep, 2017. 19(7): p. 1456-1466.

189. Omer, L., et al., CRISPR Correction of a Homozygous Low-Density Lipoprotein Receptor Mutation in Familial Hypercholesterolemia Induced Pluripotent Stem Cells. Hepatol Commun, 2017. 1(9): p. 886-898.

190. Yoshimi, K., et al., ssODN-mediated knock-in with CRISPR-Cas for large genomic regions in zygotes. Nat Commun, 2016. 7: p. 10431.

191. Yusa, K., et al., Targeted gene correction of alphal-antitrypsin deficiency in induced pluripotent stem cells. Nature, 2011. 478(7369): p. 391-4. 
192. Li, H.L., et al., Precise correction of the dystrophin gene in duchenne muscular dystrophy patient induced pluripotent stem cells by TALEN and CRISPR-Cas9. Stem Cell Reports, 2015. 4(1): p. 143-54.

193. DeWitt, M.A., J.E. Corn, and D. Carroll, Genome editing via delivery of Cas9 ribonucleoprotein. Methods, 2017. 121-122: p. 9-15.

194. DeWitt, M.A., et al., Selection-free genome editing of the sickle mutation in human adult hematopoietic stem/progenitor cells. Sci Transl Med, 2016. 8(360): p. 360 ra134.

195. Lee, K., et al., Nanoparticle delivery of Cas9 ribonucleoprotein and donor DNA in vivo induces homology-directed DNA repair. Nat Biomed Eng, 2017. 1: p. 889901.

196. Lingeman, E., C. Jeans, and J.E. Corn, Production of Purified CasRNPs for Efficacious Genome Editing. Curr Protoc Mol Biol, 2017. 120: p. 31.10.131.10.19.

197. Richardson, C.D., et al., Enhancing homology-directed genome editing by catalytically active and inactive CRISPR-Cas9 using asymmetric donor DNA. Nat Biotechnol, 2016. 34(3): p. 339-44.

198. Sander, J.D. and J.K. Joung, CRISPR-Cas systems for editing, regulating and targeting genomes. Nat Biotechnol, 2014. 32(4): p. 347-55.

199. Makarova, K.S., et al., An updated evolutionary classification of CRISPR-Cas systems. Nat Rev Microbiol, 2015. 13(11): p. 722-36. 
200. Morita, S., et al., Targeted DNA demethylation in vivo using dCas9-peptide repeat and scFv-TET1 catalytic domain fusions. Nat Biotechnol, 2016. 34(10): p. 10601065.

201. Zetsche, B., et al., Cpf1 is a single RNA-guided endonuclease of a class 2 CRISPR-Cas system. Cell, 2015. 163(3): p. 759-71.

202. Kim, D., et al., Genome-wide analysis reveals specificities of Cpf1 endonucleases in human cells. Nat Biotechnol, 2016. 34(8): p. 863-8.

203. Kleinstiver, B.P., et al., Engineered CRISPR-Cas9 nucleases with altered PAM specificities. (1476-4687 (Electronic)).

204. Waxmonsky, N. CRISPR 101: Mammalian Expression Systems and Delivery Methods. 2017 Dec 4, 2017 [cited 2018; Available from: https://blog.addgene.org/crispr-101-mammalian-expression-systems-and-deliverymethods.

205. Patrick, M. Plasmids 101: Mammalian Vectors. 2014 [cited 2018; Available from: https://blog.addgene.org/plasmids-101-mammalian-vectors.

206. Ran, F.A., et al., In vivo genome editing using Staphylococcus aureus Cas9. Nature, 2015. 520(7546): p. 186-91.

207. McDade, J. Genome engineering using Cas9/gRNA Ribonucleoproteins (RNPs). 2016 [cited 2018; Available from: https://blog.addgene.org/genome-engineeringusing-cas $9 /$ grna-ribonucleoproteins-rnps.

208. Kaufman, R.J., Regulation of $m R N A$ translation by protein folding in the endoplasmic reticulum. Trends Biochem Sci, 2004. 29(3): p. 152-8. 
209. Stevens, F.J. and Y. Argon, Protein folding in the ER. Semin Cell Dev Biol, 1999. 10(5): p. 443-54.

210. Harvey Lodish, A.B., S Lawrence Zipursky, Paul Matsudaira, David Baltimore, and James Darnell, Section 5.4, Organelles of the Eukaryotic Cell, in Molecular Cell Biology. 2000, W.H. Freeman: New York.

211. Kaufman, R.J., Stress signaling from the lumen of the endoplasmic reticulum: coordination of gene transcriptional and translational controls. Genes Dev, 1999. 13(10): p. 1211-33.

212. Zhu, G. and A.S. Lee, Role of the unfolded protein response, GRP78 and GRP94 in organ homeostasis. J Cell Physiol, 2015. 230(7): p. 1413-20.

213. Hetz, C., et al., The unfolded protein response: integrating stress signals through the stress sensor IRE1alpha. Physiol Rev, 2011. 91(4): p. 1219-43.

214. Zhang, L. and H.H. Wang, The essential functions of endoplasmic reticulum chaperones in hepatic lipid metabolism. Dig Liver Dis, 2016. 48(7): p. 709-16.

215. Dorner, A.J., et al., Protein Retention in the Endoplasmic Reticulum Mediated by GRP78, in Cell Biology and Biotechnology, R.R.G. Oka M.S., Editor. 1993, Springer: Springer, New York, NY.

216. $\mathrm{Bu}, \mathrm{G}$. and $\mathrm{S}$. Rennke, Receptor-associated protein is a folding chaperone for low density lipoprotein receptor-related protein. J Biol Chem, 1996. 271(36): p. 22218-24.

217. Gardner, B.M., et al., Endoplasmic reticulum stress sensing in the unfolded protein response. Cold Spring Harb Perspect Biol, 2013. 5(3): p. a013169. 
218. Schroder, M. and R.J. Kaufman, The mammalian unfolded protein response. Annu Rev Biochem, 2005. 74: p. 739-89.

219. Mori, K., et al., Signalling from endoplasmic reticulum to nucleus: transcription factor with a basic-leucine zipper motif is required for the unfolded proteinresponse pathway. Genes Cells, 1996. 1(9): p. 803-17.

220. Cox, J.S. and P. Walter, A novel mechanism for regulating activity of a transcription factor that controls the unfolded protein response. Cell, 1996. 87(3): p. 391-404.

221. Lee, K., et al., IRE1-mediated unconventional mRNA splicing and S2P-mediated ATF6 cleavage merge to regulate XBP1 in signaling the unfolded protein response. Genes Dev, 2002. 16(4): p. 452-66.

222. Calfon, M., et al., IRE1 couples endoplasmic reticulum load to secretory capacity by processing the XBP-1 $m R N A$. Nature, 2002. 415(6867): p. 92-6.

223. Yoshida, H., et al., XBP1 mRNA is induced by ATF6 and spliced by IRE1 in response to ER stress to produce a highly active transcription factor. Cell, 2001. 107(7): p. 881-91.

224. Yoshida, $\mathrm{H}$., et al., $p X B P 1(U)$ encoded in $X B P 1$ pre-mRNA negatively regulates unfolded protein response activator $\mathrm{pXBP} 1(S)$ in mammalian ER stress response. J Cell Biol, 2006. 172(4): p. 565-75.

225. Kakiuchi, C., et al., XBP1 induces WFS1 through an endoplasmic reticulum stress response element-like motif in SH-SY5Y cells. J Neurochem, 2006. 97(2): p. 54555. 
226. Lee, A.H., N.N. Iwakoshi, and L.H. Glimcher, XBP-1 regulates a subset of endoplasmic reticulum resident chaperone genes in the unfolded protein response. Mol Cell Biol, 2003. 23(21): p. 7448-59.

227. Acosta-Alvear, D., et al., XBP1 controls diverse cell type-and condition-specific transcriptional regulatory networks. Mol Cell, 2007. 27(1): p. 53-66.

228. Ron, D., Translational control in the endoplasmic reticulum stress response. J Clin Invest, 2002. 110(10): p. 1383-8.

229. Silva, R.M., et al., CHOP/GADD153 is a mediator of apoptotic death in substantia nigra dopamine neurons in an in vivo neurotoxin model of parkinsonism. J Neurochem, 2005. 95(4): p. 974-86.

230. Marciniak, S.J., et al., CHOP induces death by promoting protein synthesis and oxidation in the stressed endoplasmic reticulum. Genes Dev, 2004. 18(24): p. 3066-77.

231. Harding, H.P., et al., An integrated stress response regulates amino acid metabolism and resistance to oxidative stress. Mol Cell, 2003. 11(3): p. 619-33.

232. Haze, K., et al., Mammalian transcription factor ATF6 is synthesized as a transmembrane protein and activated by proteolysis in response to endoplasmic reticulum stress. Mol Biol Cell, 1999. 10(11): p. 3787-99.

233. Szegezdi, E., et al., Mediators of endoplasmic reticulum stress-induced apoptosis. EMBO Rep, 2006. 7(9): p. 880-5.

234. Goldstein, J.L. and M.S. Brown, The Ldl Receptor Defect in Familial Hypercholesterolemia - Implications for Pathogenesis and Therapy. Medical Clinics of North America, 1982. 66(2): p. 335-362. 
235. Goldstein, J.L. and M.S. Brown, The LDL receptor. Arterioscler Thromb Vasc Biol, 2009. 29(4): p. 431-8.

236. Varret, M. and J. Pierre-Rabes, Missense Mutation in the LDLR Gene: A Wide Spectrum in the Severity of Familial Hypercholesterolemia, Mutations in Human Genetic Disease, in Mutations in Human Genetic Disease, P.D. Cooper, Editor. 2012.

237. Wiegman, A., et al., Familial hypercholesterolaemia in children and adolescents: gaining decades of life by optimizing detection and treatment. Eur Heart J, 2015. 36(36): p. 2425-37.

238. Sjouke, B., et al., Homozygous autosomal dominant hypercholesterolaemia in the Netherlands: prevalence, genotype-phenotype relationship, and clinical outcome. Eur Heart J, 2015. 36(9): p. 560-5.

239. Soutar, A.K. and R.P. Naoumova, Mechanisms of disease: genetic causes of familial hypercholesterolemia. Nat Clin Pract Cardiovasc Med, 2007. 4(4): p. $214-25$.

240. Kakaei, F., et al., Liver Transplantation for Homozygous Familial Hypercholesterolemia: Two Case Reports. Transplantation Proceedings, 2009. 41(7): p. 2939-2941.

241. Kassim, S.H., et al., Adeno-associated virus serotype 8 gene therapy leads to significant lowering of plasma cholesterol levels in humanized mouse models of homozygous and heterozygous familial hypercholesterolemia. Hum Gene Ther, 2013. 24(1): p. 19-26. 
242. Takahashi, K., et al., Induction of pluripotent stem cells from adult human fibroblasts by defined factors. Cell, 2007. 131(5): p. 861-72.

243. Cayo, M.A., et al., JD induced pluripotent stem cell-derived hepatocytes faithfully recapitulate the pathophysiology of familial hypercholesterolemia. Hepatology, 2012. 56(6): p. 2163-71.

244. Fattahi, F., et al., Disease-corrected hepatocyte-like cells from familial hypercholesterolemia-induced pluripotent stem cells. Mol Biotechnol, 2013. 54(3): p. 863-73.

245. Ramakrishnan, V.M., et al., Restoration of Physiologically Responsive LowDensity Lipoprotein Receptor-Mediated Endocytosis in Genetically Deficient Induced Pluripotent Stem Cells. Sci Rep, 2015. 5: p. 13231.

246. Ran, F.A., et al., Double nicking by RNA-guided CRISPR Cas9 for enhanced genome editing specificity. Cell, 2013. 154(6): p. 1380-9.

247. Chu, V.T., et al., Increasing the efficiency of homology-directed repair for CRISPR-Cas9-induced precise gene editing in mammalian cells. Nat Biotechnol, 2015. 33(5): p. 543-8.

248. Nichols, W.W., et al., Characterization of a new human diploid cell strain, IMR90. Science, 1977. 196(4285): p. 60-3.

249. Hentze, H., et al., Teratoma formation by human embryonic stem cells: evaluation of essential parameters for future safety studies. Stem Cell Res, 2009. 2(3): p. 198-210.

250. Song, Z., et al., Efficient generation of hepatocyte-like cells from human induced pluripotent stem cells. Cell Res, 2009. 19(11): p. 1233-42. 
251. Ho, C.M., et al., Use of indocyanine green for functional assessment of human hepatocytes for transplantation. Asian Journal of Surgery, 2012. 35(1): p. 9-15.

252. Ran, F.A., et al., Genome engineering using the CRISPR-Cas9 system. Nat Protoc, 2013. 8(11): p. 2281-308.

253. Rodin, S., et al., Monolayer culturing and cloning of human pluripotent stem cells on laminin-521-based matrices under xeno-free and chemically defined conditions. Nat Protoc, 2014. 9(10): p. 2354-68.

254. Russell, D.W., et al., The Ldl Receptor in Familial Hypercholesterolemia - Use of Human Mutations to Dissect a Membrane-Protein. Cold Spring Harbor Symposia on Quantitative Biology, 1986. 51: p. 811-819.

255. Wilson, J.M., et al., Temporary amelioration of hyperlipidemia in low density lipoprotein receptor-deficient rabbits transplanted with genetically modified hepatocytes. Proc Natl Acad Sci U S A, 1990. 87(21): p. 8437-41.

256. Pakkanen, T.M., et al., Enhanced plasma cholesterol lowering effect of retrovirusmediated LDL receptor gene transfer to WHHL rabbit liver after improved surgical technique and stimulation of hepatocyte proliferation by combined partial liver resection and thymidine kinase--ganciclovir treatment. Gene Ther, 1999. 6(1): p. 34-41.

257. Cyranoski, D., CRISPR gene-editing tested in a person for the first time. Nature, 2016. 539(7630): p. 479.

258. Wesolowska, A., et al., Successful transplantation of hepatocytes requires temporary elimination of scavenger and NK cells, partial hepatectomy and ligation of bile duct. Ann Transplant, 2004. 9(4): p. 40-2. 
259. Tabebordbar, M., et al., In vivo gene editing in dystrophic mouse muscle and muscle stem cells. Science, 2016. 351(6271): p. 407-11.

260. Pankowicz, F.P., et al., Reprogramming metabolic pathways in vivo with CRISPR/Cas9 genome editing to treat hereditary tyrosinaemia. Nat Commun, 2016. 7: p. 12642.

261. Cyranoski, D., Chinese scientists to pioneer first human CRISPR trial. Nature, 2016. 535(7613): p. 476-7.

262. Puppi, J., et al., Improving the techniques for human hepatocyte transplantation: report from a consensus meeting in London. Cell Transplant, 2012. 21(1): p. 1-10.

263. Martin, F., et al., Biased and Unbiased Methods for the Detection of Off-Target Cleavage by CRISPR/Cas9: An Overview. Int J Mol Sci, 2016. 17(9).

264. American Liver Foundation. Your Liver [cited 2018; Available from: https://liverfoundation.org/for-patients/about-the-liver/.

265. Busuttil, R.W., Transplantation of the liver, G.B. Klintmalm, Editor. 2005.

266. Jorns, C., et al., Hepatocyte transplantation for inherited metabolic diseases of the liver. J Intern Med, 2012. 272(3): p. 201-23.

267. Oldhafer, F., et al., Immunological aspects of liver cell transplantation. World J Transplant, 2016. 6(1): p. 42-53.

268. Alkofer, B.J., et al., Liver transplant combined with heart transplant in severe heterozygous hypercholesterolemia: Report of the first case and review of the literature. Transplantation Proceedings, 2005. 37(5): p. 2250-2252. 
269. Santos, R.D., What are we able to achieve today for our patients with homozygous familial hypercholesterolaemia, and what are the unmet needs? Atherosclerosis Supplements, 2014. 15(2): p. 19-25.

270. Huppert, S.S. and K.M. Campbell, Emerging advancements in liver regeneration and organogenesis as tools for liver replacement. Curr Opin Organ Transplant, 2016. 21(6): p. 581-587.

271. Mitaka, T., The current status of primary hepatocyte culture. International Journal of Experimental Pathology, 1998. 79(6): p. 393-409.

272. Shulman, M. and Y. Nahmias, Long-Term Culture and Coculture of Primary Rat and Human Hepatocytes. Methods Molecular Biology, 2013. 945: p. 287-302.

273. Allen, K.J. and N.E. Buck, Clinical application of hepatocyte transplantation: what are the current limitations? Current Opinion in Organ Transplantation, 2006. 11(6): p. 648-653.

274. Fox, I.J. and J. Roy-Chowdhury, Hepatocyte transplantation. J Hepatol, 2004. 40(6): p. 878-86.

275. Habibullah, C.M., et al., Human fetal hepatocyte transplantation in patients with fulminant hepatic failure. Transplantation, 1994. 58(8): p. 951-2.

276. Nagata, H., et al., Prolonged survival of porcine hepatocytes in cynomolgus monkeys. Gastroenterology, 2007. 132(1): p. 321-9.

277. Follenzi, A., et al., Role of bone marrow transplantation for correcting hemophilia A in mice. Blood, 2012. 119(23): p. 5532-42. 
278. Merlin, S., et al., Kupffer Cell Transplantation in Mice for Elucidating Monocyte/Macrophage Biology and for Potential in Cell or Gene Therapy. Am J Pathol, 2016. 186(3): p. 539-51.

279. Viswanathan, P., et al., Thalidomide promotes transplanted cell engraftment in the rat liver by modulating inflammation and endothelial integrity. J Hepatol, 2016. 65(6): p. 1171-1178.

280. Han, B., et al., Cellular loss after allogenic hepatocyte transplantation. Transplantation, 2009. 87(1): p. 1-5.

281. Kerr, A.G., et al., Episomal Nonviral Gene Therapy Vectors Slow Progression of Atherosclerosis in a Model of Familial Hypercholesterolemia. Mol Ther Nucleic Acids, 2016. 5(11): p. e383.

282. Li, J., et al., In vivo gene therapy for hyperlipidemia: phenotypic correction in Watanabe rabbits by hepatic delivery of the rabbit $L D L$ receptor gene. $\mathrm{J}$ Clin Invest, 1995. 95(2): p. 768-73.

283. Marshall, E., Gene therapy death prompts review of adenovirus vector. Science, 1999. 286(5448): p. 2244-5.

284. Somanathan, S., et al., AAV vectors expressing LDLR gain-of-function variants demonstrate increased efficacy in mouse models of familial hypercholesterolemia. Circ Res, 2014. 115(6): p. 591-9.

285. KD, W., et al., MicroRNA profiling of human-induced pluripotent stem cells. Stem Cells and Development, 2009. 18(5): p. 749-58. 
286. $\mathrm{MH}, \mathrm{C} .$, et al., Induced pluripotent stem cells and embryonic stem cells are distinguished by gene expression signatures. Cell Stem Cell, 2009. 5(1): p. 11123.

287. Avior, Y., I. Sagi, and N. Benvenisty, Pluripotent stem cells in disease modelling and drug discovery. Nat Rev Mol Cell Biol, 2016. 17(3): p. 170-82.

288. Doudna, J.A. and E. Charpentier, Genome editing. The new frontier of genome engineering with CRISPR-Cas9. Science, 2014. 346(6213): p. 1258096.

289. Si-Tayeb, K., et al., Highly Efficient Generation of Human Hepatocyte-like Cells from Induced Pluripotent Stem Cells. Hepatology, 2011. 51(1): p. 297-305.

290. SK, M. and D. SA., Differentiation of hepatocytes from pluripotent stem cells. Current Protocols in Stem Cell Biology, 2013.

291. P, L., et al., Requirement for Wnt3 in vertebrate axis formation. Nature Genetics, 1999. 22(4): p. 361-65.

292. K, W. and N. R, Wnt Proteins. Cold Spring Harbor Perspectives in Biology, 2012. 4(9).

293. Lindsley, R.C., et al., Canonical Wnt signaling is required for development of embryonic stem cell-derived mesoderm. Development, 2006(133): p. 3787-96.

294. DC, H., et al., Highly efficient differentiation of hESCs to functional hepatic endoderm requires ActivinA and Wnt3a signaling. Proceedings National Academy of Sciences of the United States of America, 2008. 105(34): p. 12301-6.

295. Sullivan, G.J., et al., Generation of Functional Human Hepatic Endoderm from Human iPS cells. Hepatology, 2010. 51(1): p. 329-335. 
296. Kim, P.T.W. and C.J. Ong, Differentiation of Definitive Endoderm from Mouse Embryonic Stem Cells, in Results and Problems in Cell Differentiation. 2012, Springer, Berlin, Heidelberg.

297. Schopperle, W.M. and W.C. DeWolf, The TRA-1-60 and TRA-1-81 Human Pluripotent Stem Cell Markers Are Expressed on Podocalyxin in Embryonal Carcinoma. Stem Cells, 2009.

298. Mfopou, J.K., et al., Efficient definitive endoderm induction from mouse embryonic stem cell adherent cultures: A rapid screening model for differentiation studies. Stem Cell Research, 2014. 12(1): p. 166-177.

299. Takahashi, Y., et al., A Refined Culture System for Human Induced Pluripotent Stem Cell-Derived Intestinal Epithelial Organoids. Stem Cell Reports, 2018. 10(1): p. 314-328.

300. Wang, Y., et al., Defined and Scalable Generation of Hepatocyte-like Cells from Human Pluripotent Stem Cells. Journal of Visualized Experiments, 2017. 121.

301. Pimton, P., et al., Hypoxia enhances differentiation of mouse embryonic stem cells into definitive endoderm and distal lung cells. Stem cells and development, 2015. 24(5): p. 663-676.

302. DC, H., et al., Efficient differentiation of hepatocytes from human embryonic stem cells exhibiting markers recapitulating liver development in vivo. Stem Cells, 2008. 26(4): p. 894-902.

303. Polykandriotis, E., et al., To matrigel or not to matrigel. The American journal of pathology, 2008. 172(5): p. 1441-1442. 
304. K, C., et al., Recombinant Laminins Drive the Differentiation and SelfOrganization of hESC-Derived Hepatocytes. Stem Cell Reports, 2015. 5(6 ): p. $1250-62$.

305. Taylor-Weiner, H., A.J. Schwarzbauer Je Fau - Engler, and A.J. Engler, Defined extracellular matrix components are necessary for definitive endoderm induction. (1549-4918 (Electronic)).

306. Takayama, K., et al., 3D spheroid culture of hESC/hiPSC-derived hepatocyte-like cells for drug toxicity testing. (1878-5905 (Electronic)).

307. Li, Y., et al., Receptor-associated protein facilitates proper folding and maturation of the low-density lipoprotein receptor and its class 2 mutants. Biochemistry, 2002. 41(15): p. 4921-8.

308. Hay, D.C., et al., Highly efficient differentiation of hESCs to functional hepatic endoderm requires ActivinA and Wnt3a signaling. Proc Natl Acad Sci U S A, 2008. 105(34): p. 12301-6.

309. Mahammad, S. and I. Parmryd, Cholesterol depletion using methyl-betacyclodextrin. (1940-6029 (Electronic)).

310. Ramakrishnan, V.M., et al., Wnt5a Regulates the Assembly of Human Adipose Derived Stromal Vascular Fraction-Derived Microvasculatures. PLoS One, 2016. 11(3): p. e0151402.

311. Amundson, D.M. and M. Zhou, Fluorometric method for the enzymatic determination of cholesterol. J Biochem Biophys Methods, 1999. 38(1): p. 43-52.

312. Imelli, N., et al., Cholesterol is required for endocytosis and endosomal escape of adenovirus type 2. J Virol, 2004. 78(6): p. 3089-98. 
313. Williams, M.R.L.a.D.B., Calnexin and Calreticulinm Molecular Chaperones of the Endoplasmic Reticulum, in Madame Curie Bioscience Database. 2000-2013, Landes Bioscience: Austin, TX.

314. Paul N. McMillan, D.C.H., Karen A. Hevey, Sharda Naik, and Hugo O. Jauregui, Hepatocyte Cell Surface Polarity as Demonstrated by Lectin Binding. The Journal of Histochemistry and Cytochemistry, 1988. 36(12): p. 1561-1571.

315. Zhijian Wu, E.M., Mavis Agbandje-McKenna, and Richard Jude Samulski, $\alpha 2,3$ and a2,6 N-Linked Sialic Acids Facilitate Efficient Binding and Transduction by Adeno-Associated Virus Types 1 and 6. Journal of Virology, 2006. 80(18): p. 9093-9103.

316. Murray, S.S., et al., The effects of lovastatin on proteasome activities in highly purified rabbit 20 S proteasome preparations and mouse MC3T3-E1 osteoblastic cells. Metabolism, 2002. 51(9): p. 1153-60.

317. Sharmila Rao, D.C.P., Xiaomei Chen, Thaddeus Herliczek, Michael Lowe, and Khandan Keyomarsi, Lovastatin-mediated G1 arrest is through inhibition of the proteasome, independent of hydroxymethyl glutaryl-CoA reductase. . Proceedings of the National Academy of Sciences of the United States of America, 1999. 96(14): p. 7797-802.

318. Wojcik, C., et al., Lovastatin and simvastatin are modulators of the proteasome. Int J Biochem Cell Biol, 2000. 32(9): p. 957-65.

319. Park, H., et al., Plasma membrane cholesterol is a key molecule in shear stressdependent activation of extracellular signal-regulated kinase. J Biol Chem, 1998. 273(48): p. 32304-11. 
320. Melman, L., et al., Proteasome regulates the delivery of LDL receptor-related protein into the degradation pathway. Mol Biol Cell, 2002. 13(9): p. 3325-35.

321. Brown, M.S. and J.L. Goldstein, Regulation of the activity of the low density lipoprotein receptor in human fibroblasts. Cell, 1975. 6(3): p. 307-16.

322. Asselah, T., et al., In vivo hepatic endoplasmic reticulum stress in patients with chronic hepatitis $C$. The Journal of Pathology, 2010. 221(3): p. 264-274.

323. Russo, M.W., et al., Spectrum of statin hepatotoxicity: experience of the druginduced liver injury network. Hepatology (Baltimore, Md.), 2014. 60(2): p. 679686.

324. Travers, K.J., et al., Functional and Genomic Analyses Reveal an Essential Coordination between the Unfolded Protein Response and ER-Associated Degradation. Cell, 2000. 101(3): p. 2490258.

325. Soufi, M., et al., The impact of severe LDL receptor mutations on SREBPpathway regulation in homozygous familial hypercholesterolemia $(\mathrm{FH})$. Gene, 2012. 499(1): p. 218-222.

326. Ji, C. and N. Kaplowitz, ER stress: can the liver cope? J Hepatol, 2006. 45(2): p. 321-33.

327. Rutherford, J.D., Maternal heterozygous familial hypercholesterolemia and its consequences for mother and child. Circulation, 2011. 124(15): p. 1599-601.

328. Eapen, D.J., et al., Management of familial hypercholesterolemia during pregnancy: case series and discussion. J Clin Lipidol, 2012. 6(1): p. 88-91. 


\section{CURRICULUM VITAE}

NAME:

ADDRESS:

EDUCATION

\& TRAINING:
Linda Omer

Cardiovascular Innovation Institute

University of Louisville School of Medicine

302 E Muhammad Ali Boulevard

Suite 512/513

Louisville, KY 40202

B.S., Biology

Chicago State University

2009-2011

M.S., Molecular and Microbial Biology

Chicago State University

2011-2014

M.S., Biochemistry and Molecular Genetics University of Louisville 2013-2015

Ph.D., Biochemistry and Molecular Genetics University of Louisville 2015-2018

AWARDS \& HONORS:

Magna Cum Laude

Chicago State University, Chicago

2011

Merit Award

Chicago State University, Chicago

2011

Dean's List

Chicago State University, Chicago

2010-2011 
SCHOLARSHIPS

\& FELLOWSHIPS: Integrated Programs in the Biomedical Sciences (IPIBS) Graduate Fellowship

2013-2015

PROFESSIONAL SOCIETIES:
American Society for Biochemistry and Molecular Biology 2017-present

American Association for the Study of Liver Diseases 2017-present

American Heart Association 2016-2017

\section{PUBLICATIONS:}

1. Linda Omer, Elizabeth A. Hudson, Shirong Zheng, James B. Hoying, Yuan Shan, and Nolan L. Boyd. "CRISPR correction of a homozygous low-density lipoprotein receptor mutation in familial hypercholesterolemia induced pluripotent stem cells." Hepatology Communications. 16Oct2017. doi: $10.1002 /$ hep4.1110

2. Douglas Saforo, Linda Omer, Andrei Smolenkov, Aditya Barve, Lavona Casson, Nolan L Boyd, Geoffrey Clark, Leah Siskind, Levi Beverly. "Primary lung cancer samples cultured under microenvironment-mimetic conditions enrich for mesenchymal stem-like cells that promote metastasis." (Submitted).

3. Linda Omer, Lubna Hindi, Giuseppe Militello, Kenneth C. Tien, and Nolan L. Boyd. "Statin-Induced Accumulation of Class II Immature LDLReceptor in Familial Hypercholesterolemia Does Not Activate the Unfolded Protein Response.” (In Preparation)

4. Linda Omer, Lubna Hindi, Nolan L. Boyd. "Mammalian Species Wnt3a Differential Modulation of Derived-Definitive Endoderm." (In Preparation)

5. Linda Omer and Nolan L. Boyd. "Gene Therapy in Familial Hypercholesterolemia: A Journey from Past to Present” (In Preparation) 


\section{ABSTRACTS}

\section{ORAL PRESENTATIONS:}

1. Linda Omer, Hiren R. Patel, Praneeth Goli, Jacob R. Dale, Jason E. Beare, Jay B. Hoying, Terry M. McCurry, Tarakasipavan S. Grandhi, Kaushal Rege, and Nolan L. Boyd. "Liver Organoid Vascularization Using Adipose Microvessel Fragments.” 2016 Biofabrication Conference. Winston-Salem, NC (October 29-31).

2. Linda Omer, Elizabeth A. Hudson, Shirong Zheng, Jay B. Hoying, and Nolan L. Boyd. "CRISPR Correction of Low-Density Lipoprotein Receptor Mutation in Homozygous Familial HypercholesterolemiaPatient-Derived Induced Pluripotent Stem Cells Using CRISPR/Cas9 Genome Editing.” 2017 Biochemistry and Molecular Genetics Retreat. Louisville, KY (August 18).

3. Linda Omer, Elizabeth A. Hudson, Shirong Zheng, Jay B. Hoying, and Nolan L. Boyd. "CRISPR Genome Editing to Correct LDLR Mutations." 2017 Department of Biochemistry and Molecular Genetics Research Conference. Louisville, KY (August 30).

\section{POSTER PRESENTATIONS:}

1. Linda Omer and Rong L. He. "A-SAA Can Be Considered a Chemokine.” 2012 S.T.E.M Conference. Chicago, IL (February 3February 4).

2. Linda Omer and Rong L. He. "A-SAA Can Be Considered a Chemokine.” 2012 DTRA/NSF/NGA Algorithm Workshop. San Diego, CA (November 26- November 29).

3. Linda Omer and Rong L. He. "Finding New Receptors for serum amyloid A.” 2013 Immunology 2013 Conference. Honolulu, HI (May 3 May 7).

4. Linda Omer, Elizabeth A. Hudson, Hiren R. Patel, Jay B. Hoying, and Nolan L. Boyd. "CRISPR/Cas9 Correction of Class 2 Low-Density Lipoprotein Receptor- Deficiency in Homozygous Familial Hypercholesterolemia Induced Pluripotent Stem Cells." 2016 Research Louisville Conference. Louisville, KY (October 11-14).

5. Linda Omer, Hiren R. Patel, Praneeth Goli, Jacob R. Dale, Jason E. Beare, Jay B. Hoying, Terry M. McCurry, Tarakasipavan S. Grandhi, Kaushal Rege, and Nolan L. Boyd. "Liver Organoid Vascularization 
Using Adipose Microvessel Fragments.” 2016 Biofabrication Conference. Winston-Salem, NC (October 29-31).

6. Linda Omer, Elizabeth A. Hudson, Jay B. Hoying, and Nolan L. Boyd. "CRISPR/Cas9 Genome Editing to Repair Receptor-Mediated Endocytosis in Homozygous Familial Hypercholesterolemia Induced Pluripotent Stem Cells.” 2017 Experimental Biology Conference. Chicago, IL (April 22-26).

7. Linda Omer, Elizabeth A. Hudson, Shirong Zheng, Jay B. Hoying, and Nolan L. Boyd. "CRISPR Correction of Low-Density Lipoprotein Receptor Mutation in Homozygous Familial HypercholesterolemiaPatient-Derived Induced Pluripotent Stem Cells Using CRISPR/Cas9 Genome Editing." 2017 Biochemistry and Molecular Genetics Retreat. Louisville, KY (August 18).

8. Linda Omer, Elizabeth A. Hudson, Shirong Zheng, Jay B. Hoying, and Nolan L. Boyd. "CRISPR Correction of Low-Density Lipoprotein Receptor Mutation in Homozygous Familial Hypercholesterolemia Induced Pluripotent Stem Cells." Poster Presentation. 2016 Research Louisville Conference. Louisville, KY (September 12-13).

9. Shirong Zheng, Braden Bocard, Linda Omer, and Nolan L. Boyd. "Generation of Induced Pluripotent Stem Cells from Human Blood." Poster Presentation. 2017 Research Louisville Conference. Louisville, KY (September 12-13).

10. Christopher S. Hanson, Linda Omer, Shirong Zheng, Braden Bocard, M.L. Hassan, and Nolan L. Boyd. "CRISPR/Cas9 Point Mutation of LowDensity Lipoprotein Receptor to Decrease Binding Affinity to PCSK9." Poster Presentation. 2017 Research Louisville Conference. Louisville, KY (September 12-13).

11. Linda Omer and Nolan L. Boyd. "LDLR Processing Dysregulation by Statin Treatment of Class II Transport Mutant Cells." Poster Presentation. 2018 Experimental Biology Conference. San Diego, CA (April 21-25).

12. Lubna Hindi, Linda Omer, Nisha Patel, Kenneth Tien, and Nolan L. Boyd. "Characterization of Cholesterol Metabolism and ER Stress Activation in a Familial Hypercholesterolemia and CRISPR-Corrected Cell Line Model." Poster Presentation. 2018 Research Louisville Conference. Louisville, KY (October 9-12). 